University of Tennessee Health Science Center

UTHSC Digital Commons

\title{
Role of Medial Prefrontal Cortical Group II Metabotropic Glutamate Receptor in the Development of Cocaine Sensitization
}

Xiaohu Xie

University of Tennessee Health Science Center

Follow this and additional works at: https://dc.uthsc.edu/dissertations

Part of the Medical Neurobiology Commons, Medicinal and Pharmaceutical Chemistry Commons, Neurosciences Commons, and the Substance Abuse and Addiction Commons

\section{Recommended Citation}

Xie, Xiaohu , "Role of Medial Prefrontal Cortical Group II Metabotropic Glutamate Receptor in the Development of Cocaine Sensitization" (2007). Theses and Dissertations (ETD). Paper 298. http://dx.doi.org/10.21007/etd.cghs.2007.0360. 


\title{
Role of Medial Prefrontal Cortical Group II Metabotropic Glutamate Receptor in the Development of Cocaine Sensitization
}

\begin{abstract}
The current studies examined the role of medial prefrontal cortical (mPFC) group II metabotropic glutamate receptors ( $\mathrm{mGluR}_{2 / 3}$ ) in the development of cocaine sensitization. Initial studies demonstrated that intra-mPFC injection of the $\mathrm{mGluR}_{2 / 3}$ receptor agonist, $A P D C$, dose-dependently reduced acute behavioral response to cocaine $(0.015-15 \mathrm{nmol} /$ side with significant effects starting at $1.5 \mathrm{nmol} / \mathrm{side})$. The effects of APDC were prevented by intra-mPFC co-injections of an mGluR2/3 antagonist, LY341495 $(1.5 \mathrm{nmol} / \mathrm{side})$. Repeated intra-mPFC APDC $(1.5 \mathrm{nmol} / \mathrm{side})$ injections also prevented the initiation of behavioral and neurochemical sensitization, which is defined as enhanced nucleus accumbens (NAc) dopamine response to cocaine. Once sensitization was induced, however, intra-mPFC administration of APDC did not block cocaine-induced behavioral and neurochemical responses in sensitized animals after 7 days and 30 days withdrawal. In contrast, intra-mPFC injections of APDC were found to block the expression of behavioral and neurochemical sensitization in sensitized animals after 1 day withdrawal. Additional microdialysis studies demonstrated that intra-mPFC infusions of LY341495 increased glutamate levels in the mesocorticolimbic brain regions of control animals, while this response was enhanced in sensitized animals following short term withdrawal from repeated cocaine exposure. The mesocorticolimbic brain regions examined in these studies include the MPFC, NAc and VTA, which are well known brain regions involved in cocaine sensitization. Nonetheless, this effect was no longer apparent in animals after prolonged withdrawal (7 days and 30 days). Furthermore, additional results demonstrated that repeated cocaine exposure enhanced the vesicular ( $\mathrm{K}^{+}$evoked) and non-vesicular (cystine evoked) glutamate release in the mPFC. It was shown that LY379268, an mGluR2/3 receptor

agonist, inhibited the $\mathrm{K}^{+}$induced glutamate release and cystine induced glutamate release in the mPFC of animals following 1 day of withdrawal from repeated cocaine injections. In contrast to these data, LY 379268 did not inhibit the release of glutamate from one of these sources in the mPFC of animals following 7 day of withdrawal. Collectively, these data suggested that the mPFC mGluR2/3 receptor can reduce the motor response to cocaine. The development of cocaine sensitization may be associated with an initial increased responsiveness of the $\mathrm{MPFC}$ mGluR $_{2 / 3}$ receptor, coupled with enhanced glutamate transmission in the mPFC. Following prolonged withdrawal, loss of inhibitory control of the glutamate release within $\mathrm{mPFC}$ by the $\mathrm{mGluR}_{2 / 3}$ receptor thereafter may result in the enhanced excitatory drive, which in turn generates increased excitatory output from $\mathrm{MPFC}$ to subcortical regions including NAc and VTA.
\end{abstract}

Document Type

Dissertation

Degree Name

Doctor of Philosophy (PhD)

Program

Pharmacology

Research Advisor

Jeffery D.Steketee, Ph.D. 


\section{Keywords}

Cocaine, Behavioral sensitization, Group II metabotropic glutamate receptor, Medial prefrontal cortex

\section{Subject Categories}

Medical Neurobiology | Medicinal and Pharmaceutical Chemistry | Medicine and Health Sciences | Mental and Social Health | Neurosciences | Pharmacy and Pharmaceutical Sciences | Substance Abuse and Addiction 
ROLE OF MEDIAL PREFRONTAL CORTICAL GROUP II

METABOTROPIC GLUTAMATE RECEPTOR IN THE DEVELOPMENT OF

COCAINE SENSITIZATION

\author{
A Dissertation \\ Presented for \\ The Graduate Studies Council \\ The University of Tennessee \\ Health Science Center
}

In Partial Fulfillment

Of the Requirements for the Degree

Doctor of Philosophy

From The University of Tennessee

By

Xiaohu Xie

December, 2007 
Copyright (C) 2007 by Xiaohu Xie

All rights reserved 


\section{DEDICATION}

This dissertation is dedicated to my loving wife

Lan Feng.

Without her unconditional love and support

This work would not have been possible. 


\section{ACKNOWLEDGEMENTS}

I would first like to thank my advisor, Dr. Jeffery Steketee, for his guidance, encouragement and patience in leading me through the completion of this research project. I will always value his advice, honesty and friendship, and it has truly been a pleasure working with him. I would also like to thank the members of my research committee: Drs. Andrea Elberger, Burt Sharp and Fu-Ming Zhou and Steven Tavalin. Your immensely fair and constructive critiques and invaluable contributions have been much appreciated. Special thanks are also extended to the National Institute on Drug Abuse for generous support by NIH grants (DA 13470, DA15965) awarded by Dr. Steketee.

The faculty and staff of the Department of Pharmacology at University of Tennessee Health Science Center, especially the laboratories of Drs. Burt Sharp and Shannon Matta, are extended sincere thanks for helping me overcome experimental challenges. The technical microdialysis experience and friendship of Dr. Prathiba Jayaram and Victoria Kane, as well as that of Tim Walsh of Dr. Steketee's laboratory were a great aid in the completion of this work, as were the many enlightening and often comical discussions.

I am eternally thankful for my parents. You will never know how much you love and support have meant to me. Finally, words do not adequately express how grateful I am for my wife and love of my wife, Lan. Your unconditional love, encouragement and devotion are the reason this work was completed. I could not find a finer person with whom to share this moment, and I will forever be in your debt. Thank you. 


\begin{abstract}
The current studies examined the role of medial prefrontal cortical (mPFC) group II metabotropic glutamate receptors $\left(\mathrm{mGluR}_{2 / 3}\right)$ in the development of cocaine sensitization. Initial studies demonstrated that intra-mPFC injection of the $\mathrm{mGluR}_{2 / 3}$ receptor agonist, APDC, dose-dependently reduced acute behavioral response to cocaine $(0.015-15 \mathrm{nmol} / \mathrm{side}$ with significant effects starting at $1.5 \mathrm{nmol} / \mathrm{side})$. The effects of APDC were prevented by intra-mPFC co-injections of an $\mathrm{mGluR}_{2 / 3}$ antagonist, LY341495 (1.5 nmol/side). Repeated intra-mPFC APDC (1.5 nmol/side) injections also prevented the initiation of behavioral and neurochemical sensitization, which is defined as enhanced nucleus accumbens (NAc) dopamine response to cocaine. Once sensitization was induced, however, intra-mPFC administration of APDC did not block cocaine-induced behavioral and neurochemical responses in sensitized animals after 7 days and 30 days withdrawal. In contrast, intra-mPFC injections of APDC were found to block the expression of behavioral and neurochemical sensitization in sensitized animals after 1 day withdrawal. Additional microdialysis studies demonstrated that intra-mPFC infusions of LY341495 increased glutamate levels in the mesocorticolimbic brain regions of control animals, while this response was enhanced in sensitized animals following short term withdrawal from repeated cocaine exposure. The mesocorticolimbic brain regions examined in these studies include the mPFC, NAc and VTA, which are well known brain regions involved in cocaine sensitization. Nonetheless, this effect was no longer apparent in animals after prolonged withdrawal ( 7 days and 30 days). Furthermore, additional results demonstrated that repeated cocaine exposure enhanced the vesicular $\left(\mathrm{K}^{+}\right.$ evoked) and non-vesicular (cystine evoked) glutamate release in the mPFC. It was shown that $\mathrm{LY} 379268$, an $\mathrm{mGluR}_{2 / 3}$ receptor agonist, inhibited the $\mathrm{K}^{+}$induced glutamate release and cystine induced glutamate release in the mPFC of animals following 1 day of withdrawal from repeated cocaine injections. In contrast to these data, LY 379268 did not inhibit the release of glutamate from one of these sources in the mPFC of animals following 7 day of withdrawal. Collectively, these data suggested that the $\mathrm{mPFC} \mathrm{mGluR}_{2 / 3}$ receptor can reduce the motor response to cocaine. The development of cocaine sensitization may be associated with an initial increased responsiveness of the $\mathrm{mPFC} \mathrm{mGluR}_{2 / 3}$ receptor, coupled with enhanced glutamate transmission in the mPFC. Following prolonged withdrawal, loss of inhibitory control of the glutamate release within $\mathrm{mPFC}$ by the $\mathrm{mGluR}_{2 / 3}$ receptor thereafter may result in the enhanced excitatory drive, which in turn generates increased excitatory output from $\mathrm{mPFC}$ to subcortical regions including NAc and VTA.
\end{abstract}




\section{TABLE OF CONTENTS}

Chapter 1. Introduction.....................................................................1

Definition of Drug Addiction ..........................................................................

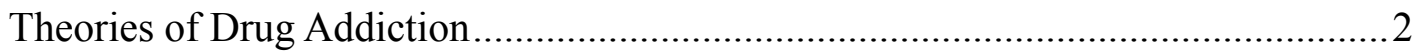

Animal Models in Drug Addiction Research .........................................................

Drug Self-Administration..............................................................................

Conditioned Place Preference ………………………....................................

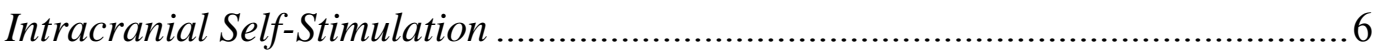

Behavioral Sensitization as a Behavioral Model in Addiction Research ....................7

Neural Basis of Behavioral Sensitization .................................................................

The Ventral Tegmental Area and the Initiation of Behavioral Sensitization.......... 9

The Nucleus Acumbens and the Expression of Behavioral Sensitization .............. 11

Other Brain Regions and Behavioral Sensitization ............................................. 12

Medial Prefrontal Cortex: Anatomy and Neurocircuitry............................................ 12

Role of Medial Prefrontal Cortex in Behavioral Sensitization..................................... 14

Role of mPFC Dopaminergic System in Sensitization .......................................... 14

Role of mPFC GABAergic System in Sensitization..............................................16

Role of mPFC Glutamatergic System in Cocaine .................................................. 17

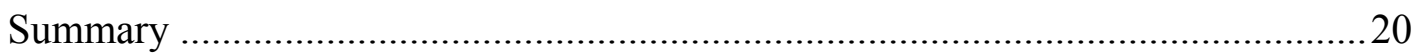

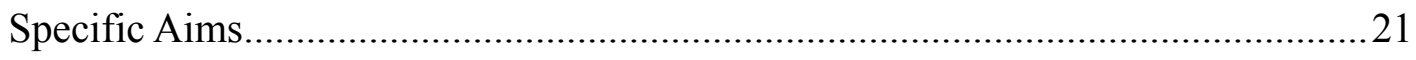

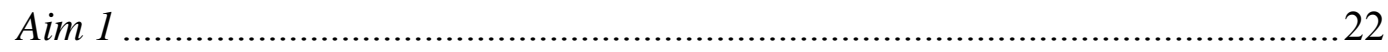

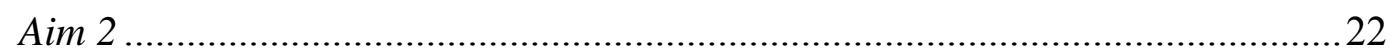

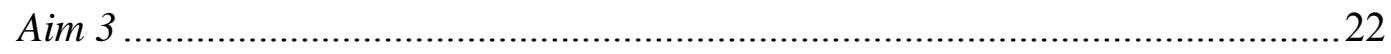

Chapter 2. Repeated Exposure to Cocaine Reduces the Ability of Medial Prefrontal Cortical Group II Metabotropic Glutamate Receptors to Modulate Behavioral and Neurochemical Responses to Cocaine .......................................................23

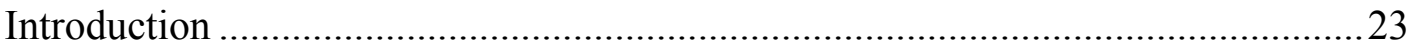

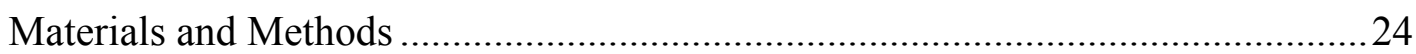

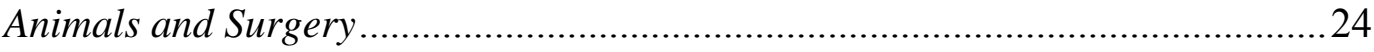

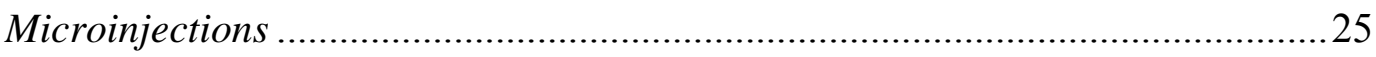

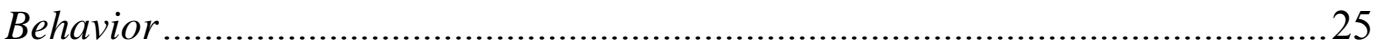

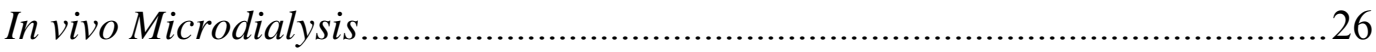

High Performance Liquid Chromatography .....................................................26

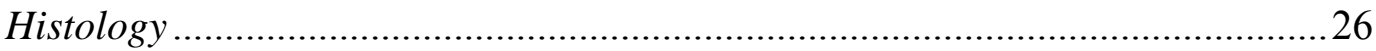

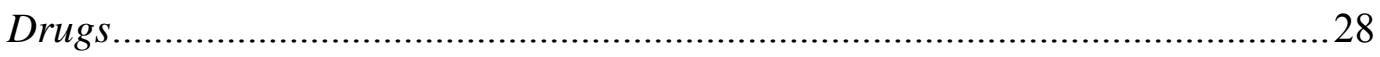

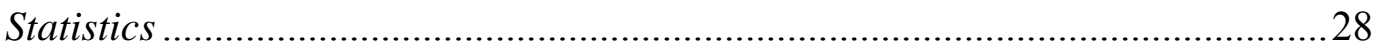

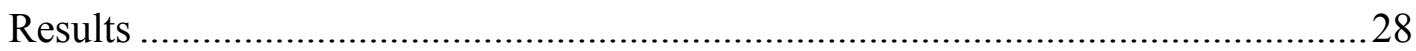

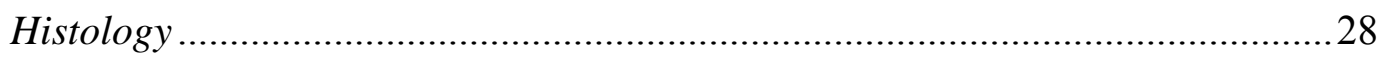

Intra-mPFC APDC and Acute Behavioral Response to Cocaine ..........................30

Intra-mPFC APDC Injections and Initiation of Cocaine Sensitization..................30 
Intra-mPFC APDC Injections and Expression of Cocaine Sensitization

Discussion

Medial Prefrontal Cortex and Acute Motor Activity

Cortical Excitatory Transmission and Behavioral Sensitization ...........................39

Cortical mGluR $R_{2 / 3}$ Receptors and Behavioral Sensitization ................................. 39

Summary

\section{Chapter 3. Repeated Exposure to Cocaine Alters the} Modulation of Mesocorticolimbic Glutamate Transmission by Medial Prefrontal Cortex Group II Metabotropic

\section{Glutamate Receptors .........................................................................................441}

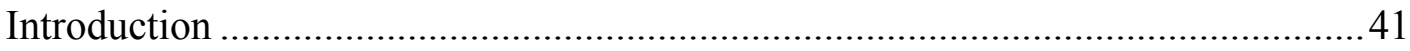

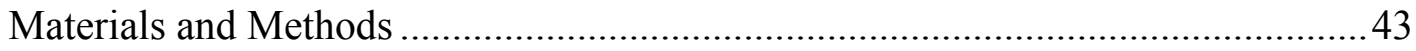

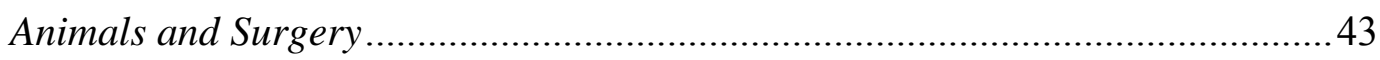

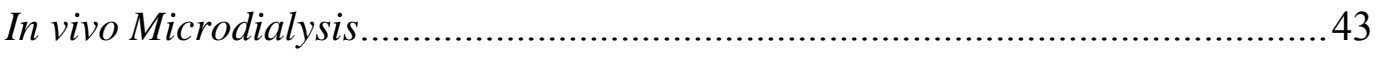

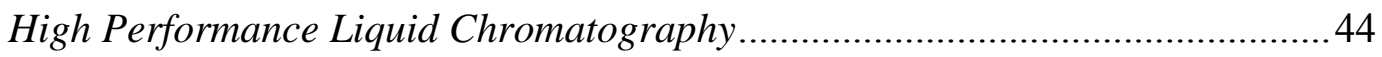

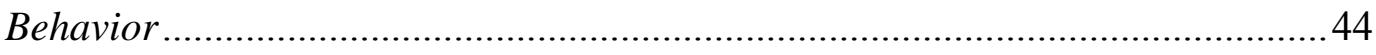

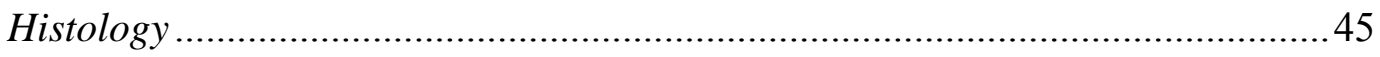

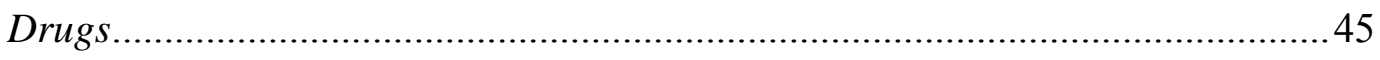

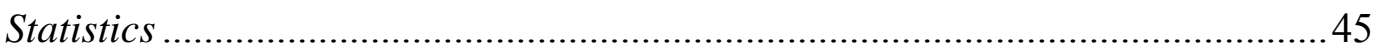

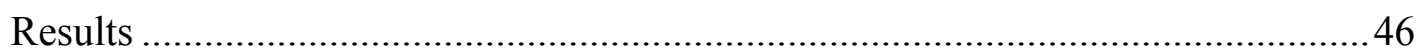

Experiment 1: GluR $_{2 / 3}$ Receptor and Mesocorticolimbic

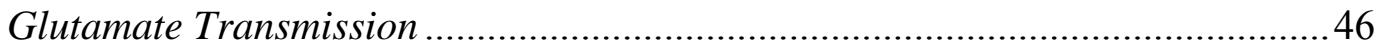

Experiment 2: $\mathrm{mGluR}_{2 / 3}$ Receptor and mPFC Glutamate Release .......................52

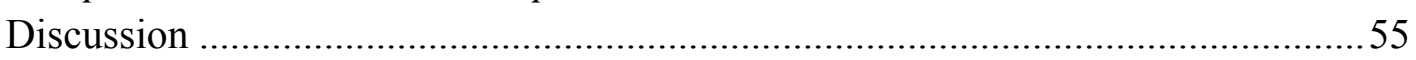

Chapter 4. General Discussion ..........................................................61

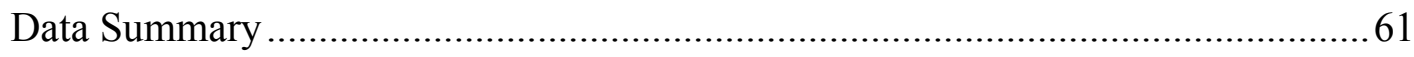

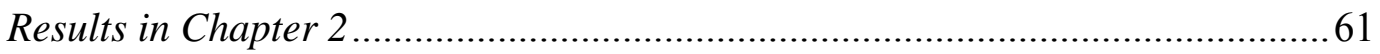

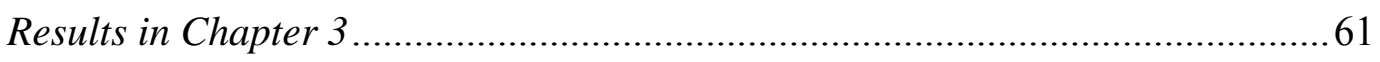

Potential Mechanisms Underlying the Alterations of Medial Prefrontal Excitatory

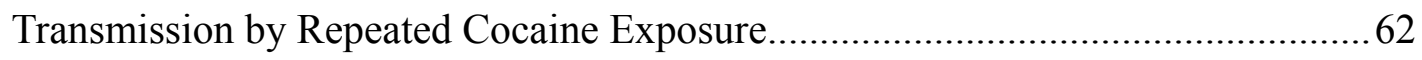

Medial Prefrontal Extracellular Glutamate Level............................................63

Medial Prefrontal Glutamate Outputs and Mesolimbic Dopamine System...........67

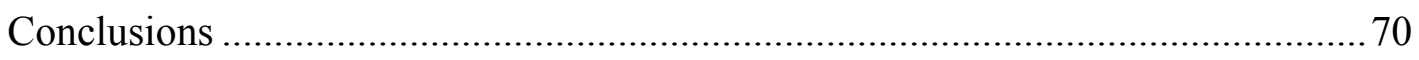

List of References .............................................................................71

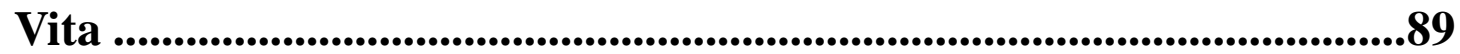




\section{LIST OF FIGURES}

Figure 2-1. Injection sites and dialysis probe placements in the mPFC and NAc......29

Figure 2-2. The effects of intra-mPFC injection of APDC on the acute motor response to cocaine $(15 \mathrm{mg} / \mathrm{kg}$ i.p. $)$.

Figure 2-3. The effects of intra-mPFC APDC (1.5 nmol/side) injection on the initiation of behavioral and neurochemical sensitization to cocaine $(15 \mathrm{mg} / \mathrm{kg}$ i.p.).

Figure 2-4. The effects of intra-mPFC APDC (1.5 nmol/side) injection on the expression of behavioral and neurochemical sensitization to cocaine $(15 \mathrm{mg} / \mathrm{kg}$ i.p.) after 1 day withdrawal.

Figure 2-5. The effects of intra-mPFC APDC (1.5 nmol/side) injection on the expression of behavioral and neurochemical sensitization to cocaine (15 mg/kg i.p.) after 7 day withdrawal.

Figure 2-6. The effects of intra-mPFC APDC (1.5 nmol/side) injection on the expression of behavioral and neurochemical sensitization to cocaine (15 mg/kg i.p.) after 30 day withdrawal. .36

Figure 3-1. Motor stimulant response to cocaine in animals that had previously received the daily repeated cocaine injections in experiment 1 .

Figure 3-2. The effects of the intra-mPFC infusion of LY341495 (0.1, 1 and $10 \mu \mathrm{M})$ on extracellular glutamate levels within the mPFC region withdrawn from repeated cocaine exposure.

Figure 3-3. The effects of the intra-mPFC infusion of LY341495 $(0.1,1$ and $10 \mu \mathrm{M})$ on extracellular glutamate levels within the NAc region withdrawn from repeated cocaine exposure. .50

Figure 3-4. The effects of the intra-mPFC infusion of LY341495 (0.1, 1 and $10 \mu \mathrm{M})$ on extracellular glutamate levels within the VTA region withdrawn from repeated cocaine exposure.

Figure 3-5. Motor stimulant response to cocaine in animals had previously received the daily repeated cocaine injections in experiment 2. 
Figure 3-6. The effects of the infusion of $\mathrm{KCl}(80 \mathrm{mM})$ into the $\mathrm{mPFC}$ on extracellular glutamate levels within this region in animals withdrawn from repeated cocaine injections.

Figure 3-7. The effects of the infusion of cystine $(50 \mathrm{uM})$ into the $\mathrm{mPFC}$ on extracellular glutamate levels within this region in animals withdrawn from repeated cocaine injections. .56

Figure 3-8. Representative micrographs of dialysis probe sites in the mPFC, NAc and VTA. 


\section{LIST OF ABBREVIATIONS}

\begin{tabular}{|c|c|}
\hline 5-HT1B & serotonergic receptor subtype $1 \mathrm{~B}$ \\
\hline 6-OHDA & 6-bydroxydopamine \\
\hline AGS3 & activator of $\mathrm{G}$ protein signaling 3 \\
\hline AMPA & $\alpha$-amino-3-hydroxy-5-methylisoxazole-4- propionic acid \\
\hline APDC & 2R, 4R-4-aminopyrrolidine-2, 4-dicarboxylate \\
\hline cAMP & cyclic adenosine monophosphate \\
\hline CPP & conditioned place preference \\
\hline CREB & cAMP response element-binding \\
\hline $\mathrm{CS}$ & conditioned stimulus \\
\hline DA & dopamine \\
\hline DCG-IV & (2S,2'R,3'R)-2-(2',3'-dicarboxycyclopropyl) glycine \\
\hline DHPG & (RS)-3,5-dihydroxyphenylglycine \\
\hline DT-CI & discrete-trial current-intensity \\
\hline EAATs & excitatory amino acid transporters \\
\hline EPSCs & excitatory postsynaptic currents \\
\hline ERK $1 / 2$ & extracellular signal-regulated kinases $1 / 2$ \\
\hline GABA & $\gamma$-aminobutyric acid \\
\hline $\mathrm{GABA}_{\mathrm{A}}$ & $\gamma$-aminobutyric acid receptor subtype A \\
\hline $\mathrm{GABA}_{\mathrm{B}}$ & $\gamma$-aminobutyric acid receptor subtype B \\
\hline HPLC & high performance liquid chromatography \\
\hline ICSS & intracranial self-stimulation \\
\hline i.p. & intraperitoneal \\
\hline IP3 & inositol trisphosphate \\
\hline IPSCs & inhibitory postsynaptic currents \\
\hline LTD & long-term depression \\
\hline LTP & long-term potentiation \\
\hline LY341495 & $\begin{array}{l}\text { (2S)-2-Amino-2-[(1S,2S)-2-carboxycycloprop-1-yl]-3-(xan th-9-yl) } \\
\text { propanoic acid }\end{array}$ \\
\hline LY379268 & $\begin{array}{l}(1 \mathrm{R}, 4 \mathrm{R}, 5 \mathrm{~S}, 6 \mathrm{R})-4-\text { Amino-2-oxabicyclo[3.1.0] } \\
\text { hexane-4,6-dic arboxylic acid }\end{array}$ \\
\hline MD & nucleus of the thalamus \\
\hline $\mathrm{mGluR}_{2 / 3}$ & Group II mGluRs \\
\hline mGluRs & metabotropic glutamate receptors \\
\hline $\mathrm{mPFC}$ & medial prefrontal cortex \\
\hline NAc & nucleus accumbens) \\
\hline NMDA & N-methyl-D-aspartate \\
\hline OXR2 & Orexin receptor type 2 \\
\hline PFC & prefrontal cortex \\
\hline PKA & protein kinase $\mathrm{A}$ \\
\hline PKC & protein kinase $\mathrm{C}$ \\
\hline
\end{tabular}


PLC phospholipase C

PN paranigral nuclei

R-FCS rate-frequency curve shift

VGKCs voltage-gated $\mathrm{K}+$ currents

VGLUT vesicular glutamate transporter proteins

VMAT vesicular monoamine transporter

VTA ventral tegmental area

WHO World Health Organization 


\section{Chapter 1. Introduction}

\section{Definition of Drug Addiction}

Drug abuse has existed to some degree throughout human history that has been recorded (Merlin 2003; Aldrich and Barker 1976). This phenomenon has been exacerbated significantly during the 20th century due to various reasons, such as agricultural improvement, biochemical advancements, increases in clinical drug usage and so on, all of which accelerated the spread of the drug addiction problem. Later on with the introduction of synthetic compounds, such as methamphetamine, drug addiction explosively became a social and medical problem.

The definitions of drug addiction were officially described as early as 1957 by the World Health Organization (WHO) Expert Committee on Addiction-Producing Drugs that defined addiction and habituation as components of drug abuse: Drug addiction is a state of periodic or chronic intoxication produced by the repeated consumption of a natural or synthetic drug. Its characteristics include: an overpowering desire or need (compulsion) to continue taking the drug and to obtain it by any means; a tendency to increase the dose; a psychic (psychological) and generally a physical dependence on the effects of the drug; and the detrimental effects on the individual and on society. Drug habituation is a condition resulting from the repeated consumption of a drug. Its characteristics include a desire (but not a compulsion) to continue taking the drug for the sense of improved well-being which it engenders; little or no tendency to increase the dose; some degree of psychic dependence on the effect of the drug, but absence of physical dependence and hence of an abstinence syndrome (withdrawal); and the detrimental effects, if any, primarily on the individual (WHO 1957).

However, confusion in the use of the terms addiction and habituation still existed until a new WHO committee suggested using the term "drug dependence" to generally describe drug abuse. Therefore, drug dependence including psychic and physical dependence were initially clarified although the committee did not clearly define dependence. It said that drug abuse was "a state of psychic dependence or physical dependence, or both, on a drug, arising in a person following administration of that drug on a periodic or continued basis." Psychic dependence was defined as a state in which "there is a feeling of satisfaction and psychic drive that requires periodic or continuous administration of the drug to produce pleasure or to avoid discomfort" (WHO 1964). Based on these definitions, drug addiction has two components: physical dependency and psychological dependency. Physical dependency occurs when a drug has been used habitually and the body has become accustomed to its effects. The person must then continue to use the drug in order to feel normal, or its absence will trigger the symptoms of withdrawal. Psychological dependency occurs when a drug has been used habitually and the mind has become emotionally reliant on its effects, either to elicit pleasure or relieve pain, and the person does not feel capable 
of functioning without it. Its absence produces intense cravings, which are often brought on or magnified by stress (WHO 1964).

These old definitions of addiction, dependence and abuse have deep impacts on recent viewpoints of drug addiction. In 2001, the American Academy of Pain Medicine, the American Pain Society, and the American Society of Addiction Medicine jointly issued "Definitions Related to the Use of Opioids for the Treatment of Pain," which defined the following terms: physical dependence, tolerance, addiction and pseudo-addiction. More specifically, addiction was defined as a primary, chronic, neurobiological disease, with genetic, psychosocial, and environmental factors influencing its development and manifestations. It is characterized by behaviors that include one or more of the following: impaired control over drug use, compulsive use, continued use despite harm, and craving. Generally, drug addiction is currently defined as a condition characterized by compulsive drug intake, craving and seeking (Goldstein 1994).

\section{Theories of Drug Addiction}

As described above, the key questions in addiction, therefore, are why some individuals undergo a transition from casual drug use to compulsive drug taking, and why drug addicts find it so difficult to quit. To answer these questions, researchers have generated theories to explain the mechanisms that underlie the development of drug addiction. The traditional theory explained that drugs are taken first because of pleasure, however, repeated drug use generates neuroadaptations leading to tolerance and dependence. The cessation of drug use is prevented by the unpleasant withdrawal symptoms. Eventually, compulsive drug taking is maintained to avoid unpleasant withdrawal symptoms. Hypotheses such as positive-negative reinforcement, opponent processes and reward allostasis are all based on this explanation (Solomon and Corbit 1973; Koob and Le Moal 1997, 2001; Robinson and Berridge 2003). However, the withdrawal theory cannot explain why addicts often relapse even after they are free from withdrawal. This limit prompted other explanations to be explored.

Recently, there is a growing viewpoint that drug addiction is a form of dysfunctional learning calling habit learning (White 1996; Schultz 2000). For example, cues that predict the availability of rewards can powerfully activate NAc-related neural circuitry in both animals and humans (Schultz 1998; Knutson et al. 2001). Furthermore, repeated exposure to drugs of abuse facilitates some forms of learning (Harmer and Phillips 1999), and can activate some of the same types of neuroadaptations in reward-related neurons involved in learning (Hyman and Malenka 2001). Abused drugs can also induce long-term potentiation (LTP) and long-term depression (LTD) in neuronal circuits associated with the addiction process, suggesting a way for the behavioral consequences of drug-taking to become reinforced by learning mechanisms (Koob and Nestler 1997). Therefore, 
drug-associated cues can trigger a desire for drug use, as well as unconscious or compulsive drug-seeking behavior, with the loss of voluntary control over drug use. This theory fits well with studies of drug-taking behavior in rats, in which the same lever is pressed again and again, a situation that strongly promotes the formation of habit learning (Robinson and Berridge 2003).

However some researchers were still not satisfied with this theory, since they thought habit learning theories still cannot explain the intrinsic motivational compulsion of addictive drugs. They argued that the compulsive pursuit of addictive drugs often happens in human drug addicts due to the lack of drug availability, a totally different situation compared with lab animals. The flexible and compulsive nature of drug-seeking behavior in the addict requires an additional motivational explanation, which prompted the rise of the incentive-sensitization theory of addiction.

The incentive-sensitization theory of addiction was first raised by Robinson and Berridge. This theory focuses on how drug cues trigger excessive incentive motivation for drugs, leading to compulsive drug seeking, drug taking, and relapse (Robinson and Berridge 1993). The principal idea of this theory is that neural circuits that mediate the attribution of incentive salience may become enduringly sensitized to specific drug effects as well as drug-associated stimuli. The drug-induced brain change is called neural sensitization, which leads psychologically to hypersensitivity to drug-related representations, causing compulsive drug seeking, drug taking, and relapse (Robinson and Berridge 2000). In pharmacology studies, the term sensitization refers to an increase in a drug effect with repeated drug administration as apposed to tolerance. There are two major classes of drug effects that are sensitized by addictive drugs: psychomotor activating effects and incentive motivational effects. Both of these drug effects are mediated at least in part by mesolimbic dopamine system associated neuronal circuitry, and therefore sensitization of these behaviors is thought to reflect reorganization and sensitization of this neural system (Robinson and Berridge 2000, 2001, 2003). These psychomotor-activating effects of addictive drugs are mediated by brain systems that overlap with those involved in brain reward pathways, such as the mesolimbic dopamine system (Robinson and Becker 1986; Robinson and Berridge 1993). In addition these behavioral effects of addictive drugs can be easily measured, therefore they provide a great way to study neurobehavioral sensitization. Many studies have suggested that there are numerous factors that can influence both the initiation and expression of neurobehavioral sensitization, including genetics, hormones, drug dose, learning and stress. It has been also shown that sensitization is produced by many different drugs of abuse, including amphetamines, cocaine, opiates, methylphenidate, ethanol, and nicotine (Robinson and Berridge 2003). Sensitization has also revealed one of the most important features of addiction due to its remarkable persistence (Paulson et al. 1991; Castner and Goldman-Rakic 1999). 


\section{Animal Models in Drug Addiction Research}

Animal models have been established to examine the neurobiological basis of drug addiction. One of the important aspects of drug abuse is the positive reinforcement, which is the major area in current drug addiction research. Therefore, currently used animal models of drug addiction mostly focus on examining the role of positive reinforcement process in acquisition, maintenance and reinstatement of drug addiction. Many widely utilized animal models are currently well established in rodents. The application of these rodent models, combined with the development of modern research techniques of neurobiology, has provided important information regarding the neurobiology of addiction. Therefore, several popular animal models used in drug addiction study are briefly reviewed below.

\section{Drug Self-Administration}

An important assumption of many drug addiction theories is that abused drugs have a reinforcing effect. This reinforcing effect, in part, is responsible for acquisition and maintenance of drug-taking behavior. The drug self-administration task provides support for this assumption. A detailed review of intravenous self-administration has been published (Kalivas et al. 2006). Drugs of abuse are readily self-administered intravenously by experimental animals. High abuse potential drugs have high self-administered liability, although not all drugs abused by humans are self-administered by experimental animals (Markou et al. 1993). As an animal model, drug self-administration can also be influenced by species, dose of the drug, the presence or absence of environmental stimuli that signal drug infusions, post-reinforcement interval, and prior drug history of the subject animals (Mello and Negus 1996; Macenski and Meisch 1998; Kuzmin and Johansson 2000; Kalivas et al. 2006). As for many studies, a given pretreatment should be considered as a potential confounding factor, because the pretreatment may affect self-administration by having nonspecific effects on behavior such as alterations of locomotion (Kalivas et al. 2006). Therefore, the influence of non-drug reinforcing effects should be assessed.

In a typical intravenous self-administration study, the animal subject receives a drug infusion by performing a discrete task. Rats are surgically prepared with an intravenous (IV) catheter. Then, in an operant conditioning chamber two response options (left lever press versus right lever press) are made available to the rat. One response is associated with IV infusion of a drug; the other response has no consequence of drug infusion. The dependent variables are the number of infusions obtained or the rate of responding during a session. The number and pattern of responding required for each infusion is determined by the schedule of reinforcement imposed by the researcher. In addition to the intravenous route of administration, the intragastric or oral route can be employed (Sanchis-Segura and Spanagel 2006). 
Most abused drugs differentially increase the rate of responding on the drug-associated lever. This model can serve as an important preclinical model for the effectiveness of pharmacotherapies to decrease drug-taking behavior (Kalivas et al. 2006; Sanchis-Segura and Spanagel 2006). In addition, by making drug availability signaled by an environmental stimulus, this model can also be used to study the importance of the response-reinforcer relationship as well as the impact of stimuli present during self-administration (Kalivas et al. 2006; Sanchis-Segura and Spanagel 2006). Recently, there has been a significant increase in interest in the role of these stimuli in the maintenance and reinstatement of self-administration (Kalivas et al. 2006; Sanchis-Segura and Spanagel 2006).

\section{Conditioned Place Preference}

Conditioned place preference (CPP) is a popular conditioning procedure in which administration of a drug is paired with one distinct environment stimulus and administration of placebo with another. CPP is aimed at measuring the ability of drugs to act as reinforcers. One major characteristic of CPP is the fact that the drug is administered by the experimenter regardless of subject's behavior. Through this process, this stimulus acquires the ability to act as a conditioned stimulus (CS). Thereafter, this CS will be able to elicit approach/avoidance behavior depending on the nature of the drug. By measuring these approach/avoidance behaviors, further insight about the drug acting as reinforcers can be gained (Bardo and Bevins 2000).

In a typical CPP experiment, a drug is injected and the subject animal is placed in a test chamber with distinctive environmental stimulus. The apparatus used in conditioning experiments consists of at least two environments that are differentiated from each other on the basis of color, texture, and/or lighting. The distinctiveness of the environments is essential for the development of conditioning. This procedure is repeated for several days. During these conditioning trials the animal develops an association between the subjective state produced by the drug and the environmental stimulus present during the drug state. When the noninjected subject animal is tested in an apparatus that contains the drug-related environmental stimulus in one compartment and neutral stimulus in another, it voluntarily moves toward the compartment containing the drug-related stimulus. This learned association between environmental stimuli and drug effect provides the basis for conditioned place preference (CPP) experiments. The animal's choice to spend more time in either environment provides a measurement of the conditioned reinforcing effect of a drug. This procedure not only permits assessment of the conditioning of drug reinforcement but also can provide indirect information regarding the positive and negative reinforcing effects of drugs (Tzschentke 1998). Many laboratories are now using this technique to study the subjective properties of drugs because CPP studies are much easier to conduct than intravenous self-administration studies (Risinger et al. 1996; Carlezon et al. 1998; Tzschentke 1998). 
There are two different CPP procedures called unbiased and biased CPP (Bardo et al. 1995; Sanchis-Segura and Spanagel 2006). As for the former, the drug injection is associated with one randomly chosen compartment and is usually counterbalanced across the subjects, whereas regarding the latter, the drug is paired with the non-preferred compartment and CPP is measured as overcoming the initial aversion for that environment. In the often used biased design, animals exhibit a preference for one of the environment prior to conditioning, which is called preconditioning phase, in which animals are allowed access to both environments. Preconditioning phase is necessary to determine the innate preference of each animal. The drug then is paired with the preferred or non-preferred environmental stimulus depending on whether the drug is assumed to produce aversive or positive reinforcing effects, respectively.

There are many factors influencing the establishment of CPP. Drugs of abuse have a differential ability to produce CPP. Generally, opiates and psychostimulants produce robust CPPs, while other drugs such as ethanol, nicotine or cannabinoids produce more inconsistent results (Cunningham et al. 1993; Tzschentke 1998; Bardo and Bevins 2000). It has been shown that drug administration before context exposure ensured a clearer preference, otherwise, when the drug administration occurs after context exposure, conditioned place aversion (CPA) rather than CPP was observed (Font et al. 2006). The number of drug-environmental stimuli pairings also influences CPP. The higher the number of pairings, the higher or the more persistent the conditioned preference observed (Bardo and Bevins 2000; Sanchis-Segura and Spanagel 2006). Conversely, extinction of a previously acquired conditioned preference can be caused by experience with the conditioned stimuli in absence of drug exposure, which is termed Pavlovian latent inhibition (Sanchis-Segura and Spanagel 2006). CPP has been widely used in conjunction with gene transfer and homologous recombination techniques to delineate the molecular basis of drug induced reinforcement (Tzschentke 1998; Sanchis-Segura and Spanagel 2006).

\section{Intracranial Self-Stimulation}

Intracranial self-stimulation (ICSS) experiments were fundamental in the establishment of the reward concept and its application to the current views of drug addictive behavior (Olds and Milner 1954). It is hypothesized that ICSS produces the direct activation of brain circuits usually activated by natural reinforcers or drugs of abuse (Esposito et al. 1984; Porrino et al. 1984). In bypassing much of the input side of these neuronal circuits, ICSS provides a unique tool to investigate the influence of various substances on reward and reinforcement processes. Therefore, ICSS has been used in psychopharmacological research to study the brain circuits mediating the rewarding effects of drugs of abuse (Sanchis-Segura and Spanagel 2006).

ICSS differs significantly from drug self-administration because in the ICSS procedure, the animal is working to directly stimulate presumed reinforcement circuits 
in the brain, and the effects of the drugs are assessed on these reward thresholds. Drugs of abuse decrease thresholds for ICSS, and there is a good correspondence between the ability of drugs to decrease ICSS thresholds and their abuse potential (Kornetsky and Esposito 1979; Kornetsky et al. 1979). It should be noted that a wide variety of ICSS-based procedures have been developed over the years, but at least two ICSS-related procedures have been extensively applied to explore the possible effects of drugs of abuse: the discrete-trial current-intensity (DT-CI) and the rate-frequency curve shift (R-FCS) (Stellar and Stellar 1985; Markou et al. 1993). It has been shown that the administration of several drugs of abuse including cocaine, amphetamine, morphine, ethanol or nicotine results in a reduction of the ICSS reward threshold in some brain areas (Kornetsky and Bain 1992; Wise 1996). On the other hand, an elevation of the same threshold has been observed in drug-dependent animals during withdrawal (Epping-Jordan et al. 1998; Cryan et al. 2003) or when administering drugs with aversive effects (Todtenkopf et al. 2004). This evidence suggested that the ICSS-based procedures may be used in identifying substances that could reduce drug reward-related processes so as to consequently reduce their consumption. However, several disadvantages have limited its usage. For example, animal surgery for implantation of electrodes is usually required along with specialized equipment. Selection of the brain site is of vital importance, since different brain regions support different rates of brain stimulation reward due to different brain circuits. In addition, animals need to be trained for several weeks to obtain stable rates of responding or stable thresholds.

\section{Behavioral Sensitization as a Behavioral Model in Addiction Research}

One of the classic drug effects observed in laboratory animals is the phenomenon that repeated administration of psychomotor stimulants, like cocaine, can generate an enduring behavioral augmentation upon subsequent drug administration after a period of withdrawal. This phenomenon was called behavioral sensitization, which was observed as early as in 1932 (Downs and Eddy 1932). In 1993, Robinson and Berridge stated that it is the progressive and persistent neuroadaptations caused by repeated drug use that take responsibility for this drug induced behavior (Robinson and Berridge 1993). Although different doses of acute administration of these drugs may result in different types of behaviors, such as exploratory and stereotype behavior, many of these behaviors can be progressively augmented after repeated and intermittent exposure (Creese and Iversen 1973; Post and Kopanda 1976; Robinson and Becker 1986; Kalivas and Duffy 1990; King et al. 1992; Kalivas and Duffy 1993). In addition, these effects can last for a very long time (Paulson et al. 1991). The reasons why behavioral sensitization has been proposed as a useful animal model for drug addiction study, such as craving and relapse are as followed: First, the behavioral effects resemble the maladaptive psychological states in human addicts (Wise and Bozarth 1987; Robinson and Berridge 1993; Schmidt and Beninger 2006). Second, 
adaptations in brain reward circuitry were implicated in human psychopathological symptoms precipitated by long-term cocaine use (Robinson and Berridge 1993; Kalivas et al. 1998; Bradberry 2007). For instance, in addicts, doses of drugs that are too low to produce any conscious experience of pleasure can cause an increase in drug-seeking behavior (Lamb et al. 1991; Foltin and Fischman 1992). Clinical observations suggested that stimuli previously associated with availability or self-administration of the drug can evoke intense subjective feelings of craving and can trigger relapse in abstinent cocaine abuse patients (Ehrman et al. 1992).

Many factors have been shown to influence the development of behavioral sensitization as described above. Most important in animal experiments, the frequency of drug administration should be considered. It has been shown that continuous infusion of cocaine may generate tolerance, while intermittent administration can produce sensitized behavioral responses (Post 1980; King et al. 1992). Therefore, many studies use such treatment schedule that consists of a repeated daily systemic injections and a following challenge injection after a period of withdrawal, (Kalivas and Duffy 1993; Sorg et al. 1997; Beyer and Steketee 2002; Steketee 2005), although single drug injection has been shown to induce behavioral sensitization (Vanderschuren et al. 1999). Besides the frequency of drug administration, the withdrawal time between the last repeated injection and the challenge injection may affect the magnitude of the behavioral sensitization. For example, cocaine challenge can induce a greater behavioral response after a long period (2-4 weeks) of withdrawal as compared with a short period (1 day) of withdrawal (Kalivas and Duffy 1993; Henry and White 1995). Also the expression of the behavioral sensitization in this study became no longer apparent after 2 months withdrawal (Henry and White 1995). While other studies showed that behavioral sensitization can persist at least for a year (Paulson et al. 1991; Castner and Goldman-Rakic 1999). Thus, the expression of behavioral sensitization may consist of long term maintenance and declining phases. Taken together, the treatment regimen can significantly affect the development of behavioral sensitization.

The development of behavioral sensitization may also be affected by the environmental context in which animals received the drug exposure (Badiani et al. 1995; Badiani et al. 2000; Crombag et al. 2000; Carey et al. 2005). Novel environments can induce elevated behavioral response in both saline animals and sensitized animals (Badiani et al. 1995). In addition to these above mentioned factors, cross-sensitization is another factor that deserves to be mentioned. Cross-sensitization occurs when previous drug history influences the development of behavioral sensitization to another drug (Vezina and Stewart 1989, 1990). Animals pretreated with other stimulants such as nicotine (Schoffelmeer et al. 2002; Collins and Izenwasser 2004) and ethanol (Lessov and Phillips 2003) can show a sensitized motor response to the subsequent cocaine challenge. Even stress has been shown to cross-sensitize to the effects of cocaine (Sorg 1992; Prasad et al. 1998; Lepsch et al. 2005). Our lab recently reported that repetitive inhalational exposure to toluene can 
produce an augmentation in motor response to cocaine (Beyer et al. 2001). Therefore, this evidence further supports that behavioral sensitization results from the neuroadaptations within the common neural substrates that are influenced by the various stimuli (Kalivas et al. 1998; Robinson and Berridge 2001).

\section{Neural Basis of Behavioral Sensitization}

Numerous studies have investigated the mechanisms of behavioral sensitization. The majority of these studies concentrated on the mesolimbic dopamine system (Steketee et al. 1992; Robinson and Berridge 1993; Pierce and Kalivas 1997; Everitt and Wolf 2002). Mesolimbic dopaminergic neurons originate in the ventral tegmental area (VTA) and project to various limbic regions such as the nucleus accumbens (NAc) (Oades and Halliday 1987) Studies have shown that the development of behavioral sensitization is correlated with activity changes within this system (Kalivas and Duffy 1990, 1993, 1993; Robinson and Berridge 1993) Through these studies, an agreement has been reached that the development of behavioral sensitization could have at least two distinct phases: initiation and expression. The initiation of behavioral sensitization is thought to occur in the VTA and is characterized by transient changes in neuronal function responsible for behavioral augmentation (Kalivas et al. 1993; Robinson and Berridge 1993; Pierce and Kalivas 1997). Expression is thought to be mediated by NAc. In this phase, the neuronal alteration arising from the initiation mediates the augmented behavioral response (Robinson and Berridge 1993; Pierce and Kalivas 1997).

\section{The Ventral Tegmental Area and the Initiation of Behavioral Sensitization}

The early functional changes of the dopaminergic neurons in the VTA have been suggested as the substrate for the initiation of the behavioral sensitization. Repeated cocaine exposure can cause a transient reduction in dopamine $\mathrm{D}_{2}$ autoreceptor sensitivity within the early days of withdrawal (Ackerman and White 1990) Since activation of $\mathrm{D}_{2}$ autoreceptor can lead to reduction of dopamine release and synthesis, as well as the reduction of dopamine neuron firing, (Wolf and Roth 1987, 1990; White 1996) repeated cocaine injections therefore resulted in enhancement in the number of spontaneously active neurons and burst neuron firing (Henry et al. 1989; Gao et al. 1998).

Besides the functional changes occurring on the dopamine neurons, the temporary neural adaptations in the neurotransmitter systems within the VTA have also been reported. For instance, repeated cocaine administration resulted in a transient enhancement in dopamine transmission within the VTA (Kalivas and Duffy 1993). Even the increased expression of tyrosine hydroxylase, the rate limiting enzyme for the synthesis of dopamine, has been reported to be associated with cocaine 
sensitization (Beitner-Johnson et al. 1991; Sorg et al. 1993). Additionally, Intra-VTA infusion of DAT(dopamine transporter)-selective reuptake inhibitor GBR12909, mimicking some of the effects of psychostimulants like cocaine, can also induce behavioral sensitization (Steketee 1998) All these studies linked VTA dopamine transmission to the development of sensitization. VTA dopamine receptor, especially $\mathrm{D}_{1}$ receptor has been shown to be important in the initiation of sensitization. For instances, blockade of the dopaminergic transmission by directly infusing $\mathrm{D}_{1}$ receptor antagonist SCH23390 into the VTA blocked the initiation of amphetamine induced sensitization (Vezina 1996), although this effect could also be due to activation of 5-HT2C receptors in the VTA (Millan et al. 2001; Navailles et al. 2007). Repeated intra-VTA infusion of $\mathrm{D}_{1}$ receptor agonist SKF 38393 induced behavioral sensitization (Pierce et al. 1996) These results suggest that activation of $D_{1}$ receptor by increased dopamine levels in the VTA is a critical component in the initiation of sensitization. In addition to the dopaminergic system, alterations of the glutamatergic system also occur. Increased AMPA receptor sensitivity and increased AMPA receptor GluR1 subunit and NMDA receptor NR1 subunit levels have been reported to be associated with cocaine sensitization (Fitzgerald et al. 1996; Zhang et al. 1997; Wolf 1998). Consistent with this, intra-VTA infusion of NMDA receptor antagonist MK-801 was shown to block the development of cocaine sensitization (Kalivas and Alesdatter 1993). Besides these two neurotransmitter systems, other neurotransmitter systems may also be involved within the VTA. Serotonergic 5-HT1B receptors have been shown to be increased in the VTA after repeated cocaine exposure (Przegalinski et al. 2003). Repeated intra-VTA infusions of the $\mu$ opioid receptor agonist morphine or DAMGO have been shown to induce behavioral sensitization (Vezina et al. 1987). In vitro application of orexin A induces potentiation of N-methyl-D-aspartate (NMDA)receptor -mediated neurotransmission via a PLC/PKC-dependent insertion of NMDA receptors in VTA dopamine neuron synapses (Borgland et al. 2006).

Moreover, in vivo administration of an orexin 1 receptor antagonist blocks locomotor sensitization to cocaine and occludes cocaine-induced potentiation of excitatory currents in VTA dopamine neurons (Borgland et al. 2006). Furthermore, signaling pathways that are indicated to be involved in the development of sensitization have been shown to be altered. For example, protein kinase C (PKC) activation has been shown to be transiently increased within the VTA (Steketee et al. 1998). Intra-VTA infusion of PKC or protein kinase A (PKA) inhibitors has been reported to block the initiation of cocaine or amphetamine induced sensitization (Steketee 1997; Tolliver et al. 1999). Studies have also reported that the levels of Gia, alpha subunit of the inhibitory G protein in the VTA were increased after repeated cocaine administration (Nestler et al. 1990). Intra-VTA infusion of G-protein uncoupling agent pertussis toxin resulted in behavioral sensitization (Steketee et al. 1991). Taken together, these data suggest that VTA is critical to the initiation of sensitization to psychostimulants. 


\section{The Nucleus Acumbens and the Expression of Behavioral Sensitization}

Repeated and intermittent i.p. injections of psychomotor stimulants such as cocaine or amphetamine generated behavioral sensitization, while repeated intra-NAc infusion of these psychomotor stimulants alone failed to induce sensitization (Kalivas and Weber 1988; Hooks et al. 1993). However, intra-NAc infusion of amphetamine elicited behavioral sensitization in previously sensitized animals (Perugini and Vezina 1994). Combined with an earlier report that lesion of the NAc with 6-OHDA resulted in a loss of cocaine or amphetamine induced locomotor activation (Kelly and Iversen 1976), this evidence directly suggested that the NAc might be critical for the expression rather than the initiation of behavioral sensitization.

Various neurochemical alterations have been reported by many studies. One of the classic neurochemical effects of repeated psychomotor stimulants exposure, such as cocaine is the enhancement of mesolimbic dopamine transmission. Repeated administration of psychomotor stimulants increased the dopamine response within the NAc upon a subsequent challenge exposure (Hurd et al. 1989; Kalivas and Duffy 1993; Heidbreder et al. 1996). This phenomenon is referred to as neurochemical sensitization in many studies and is correlated with the expression of behavioral sensitization (Robinson and Berridge 1993; Kalivas et al. 1998). However, agreement on whether chronic cocaine exposure alters the basal dopamine levels in NAc has not been achieved. (Kalivas and Duffy 1993; Chefer and Shippenberg 2002); (Weiss et al. 1992; Heidbreder et al. 1996). Similar to enhanced dopamine release, a series of in vivo microdialysis studies have revealed that cocaine induced locomotor sensitization is also correlated with an enhancement of cocaine induced glutamate release with a reduction of basal glutamate levels within the NAc (Bell and Kalivas 1996; Cornish and Kalivas 2001; Xi et al. 2002; McFarland et al. 2003). Additionally, sensitization to cocaine or amphetamine is correlated with an increase in serotonin level as well as 5-HT1B binding in the NAc (Przegalinski et al. 2003).

Besides the alterations of neurochemical level, alterations in receptor levels have also been observed in NAc of sensitized animals. For example, GluR1 levels were enhanced in NAc of cocaine sensitized animals (Churchill et al. 1999). Behavioral sensitization to cocaine is associated with increased AMPA receptor surface expression in the NAc (Boudreau and Wolf 2005). The DA-induced inhibition of AMPA receptor-mediated synaptic responses was enhanced in cocaine-treated mice, an effect that was caused by activation of $\mathrm{D}_{1 \text {-like }}$ receptors (Boudreau and Wolf 2005). In rats that developed behavioral sensitization to repeated amphetamine, metabotropic glutmate receptors (mGluRs) were altered. $\mathrm{mGluR}_{1}$ levels in the dorsal and ventral striatum were transiently enhanced while $\mathrm{mGluR}_{5}$ levels were persistently reduced in the entire striatum, which may indicate functional implications of Group I mGluRs in the regulation of behavioral sensitization to the dopamine stimulant (Mao and Wang 2001). 
Recently several novel proteins have been found to be implicated in regulating the expression of sensitization. Orexin receptor type 2 (OXR2) but not type 1 was found to be persistently up-regulated in NAc after repeated cocaine exposure (Zhang et al. 2007). OXR2 levels in the frontal cortex, the ventral tegmental area, the hippocampus, and the dorsal striatum (caudate putamen) were not altered by cocaine (Zhang et al. 2007). In contrast to chronic cocaine administration, an acute cocaine injection was insufficient to modify levels of any orexin receptor and peptide(Zhang et al. 2007). Therefore, this OXR2 up-regulation may reflect a key adaptation of limbic orexinergic transmission to chronic drug exposure and may thus be critical for the expression of behavioral sensitization. Another example is that microinjection of CART 55-102 (Cocaine- and amphetamine-regulated transcript) peptides, which are endogenous peptidergic neurotransmitters, into the NAc dose-dependently blocked the expression of locomotor sensitization produced by repeated cocaine exposure (Yoon et al. 2007). The increase of ERK $1 / 2$ phosphorylation levels in the NAc by repeated cocaine was completely blocked by CART 55-102 peptide microinjection in this site. These results suggest that CART 55-102 peptide in the NAc may play a compensatory inhibitory role in the expression of behavioral sensitization by cocaine and these effects may be mediated by its inhibition of ERK1/2 phosphorylation (Yoon et al. 2007). Finally, regulation of gene expression in NAc via two transcription factors CREB and DeltaFosB contribute to the drug induced behaviors. These factors have been well reviewed in regulating gene expression mediating drug induced behaviors (Nestler 2004).

\section{Other Brain Regions and Behavioral Sensitization}

So far, many additional brain regions have been shown to be involved in the development of behavioral sensitization to psychomotor stimulants (Bardo 1998). Initially, investigations of other brain regions were prompted by the findings that different mechanisms may exist in the development of amphetamine and cocaine sensitization. For example, intra-VTA infusion of the $\mathrm{D}_{1}$ receptor antagonist, SCH23390 attenuated the development of sensitization to amphetamine but failed to block the development of sensitization to cocaine (Vezina 1996; Steketee 1998). Intra-VTA administration of cocaine, as opposed to amphetamine, did not induce sensitization (Kalivas and Weber 1988; Vezina 1993). Therefore, several labs including our own have begun to investigate additional brain regions that may also be involved in the development of sensitization.

\section{Medial Prefrontal Cortex: Anatomy and Neurocircuitry}

The prefrontal cortex (PFC) is characterized as the cortical region that has strong reciprocal interactions with the mediodorsal nucleus of the thalamus (MD). The PFC can modulate the activity of the basal ganglia via corticostriatal pathways and receive 
inputs via the MD (Groenewegen and Uylings 2000; Tzschentke 2000; Steketee 2005). The PFC can be subdivided into medial and dorsal subregions. The medial subregion of PFC is well studied due to its close association with goal-directed behavior relevant to drug reward (Pierce and Kalivas 1997; Schultz et al. 1998; Kalivas and Nakamura 1999; Tzschentke 2001; Steketee 2003).

A series of studies using anterograde and retrograde tracing techniques in combination with immunolabeling and electron microscopy have elucidated the principal afferent and efferent projections of the mPFC. The mPFC is a part of the meoscortical system in that it receives strong dopaminergic innervation from the VTA (Thierry et al. 1973; Oades and Halliday 1987; Goldman-Rakic et al. 1989; Sesack et al. 1989; Cowan et al. 1994; Pirot et al. 1996). In addition, the mPFC receives glutamate innervation from many subcortical regions such as the thalamus, hippocampus, and the amygdala (Lindvall et al. 1977; Conde et al. 1990; Pirot et al. 1994; Jay et al. 1995; Bacon et al. 1996; Jay et al. 1996; McDonald 1996) as well as cortical glutamatergic inputs from contralateral mPFC (Conde et al. 1995; Steketee 2003).

The mPFC can be further subdivided into several distinct regions including: the infralimbic, prelimbic, dorsal and ventral anterior cingulate, and medial precentral cortical areas (Groenewegen and Uylings 2000; Ongur and Price 2000; Heidbreder and Groenewegen 2003). It should be noted that the prelimbic and infralimbic cortical areas receive the strongest dopaminergic input from VTA, as compared with other subregions of the mPFC (Thierry et al. 1973; Lindvall et al. 1977; Cowan et al. 1994; Sesack et al. 1995). Furthermore, the neuroanatomical connections of the prelimbic and infralimbic subregions of $\mathrm{mPFC}$ have been shown to be different to some degree. For instance, although both areas project to NAc, the prelimbic area tends to preferentially innervate the core of NAc, while the infralimbic area projects preferentially to the shell of NAc (Groenewegen et al. 1990). Additionally, the glutamatergic inputs from MD tend to project to the prelimbic area rather than the infralimbic area (Sesack et al. 1989; Groenewegen et al. 1990; Conde et al. 1995).

The mPFC consists of two principal neuron types: pyramidal glutamatergic neurons and GABAergic local interneurons (Goldman-Rakic et al. 1989; Cowan et al. 1994; Sesack et al. 1995). Pyramidal neurons are the major output neurons within $\mathrm{mPFC}$, which provide reciprocal innervation to other brain regions that send projections to the $\mathrm{mPFC}$, including the VTA, amygdala, thalamus, and hippocampus (Sesack et al. 1989; Cowan et al. 1994; Steketee 2003) In addition, mPFC pyramidal neurons have axon collaterals that provide innervation across cortical layers so that these pyramidal neurons form an "intranet" within mPFC. The dopaminergic efferents from VTA synapse on both the pyramidal glutamatergic neurons and GABAergic local interneurons. Dopamine has been shown to stimulate GABA release in mPFC (Sesack et al. 1989; Law-Tho et al. 1994; Grobin and Deutch 1998; Carr and Sesack 2000). Finally, it should be noted that the $\mathrm{mPFC}$ also receives GABAergic innervation 
directly from the VTA, which can directly inhibit the pyramidal neurons (Carr and Sesack 2000).

\section{Role of Medial Prefrontal Cortex in Behavioral Sensitization}

The medial prefrontal cortex (mPFC) has been well reviewed for its critical role in the development of psychomotor stimulant induced sensitization (Steketee 2003, 2005). It has been shown that repeated exposure to cocaine was associated with an increase in mPFC pyramidal neuron excitability (Dong et al. 2005; Nasif et al. 2005) Also, excitotoxic lesions of the $\mathrm{mPFC}$, including both the prelimbic and infralimbic regions, disrupted the induction of sensitization to cocaine ( $\mathrm{Li}$ et al. 1999).

Furthermore, discrete excitotoxic lesions of the prelimbic region of the mPFC also blocked the induction of cocaine-induced sensitization (Tzschentke and Schmidt 1998, 2000). Once sensitization had been induced, ibotenic acid lesions of the mPFC failed to prevent the expression of cocaine induced sensitization ( $\mathrm{Li}$ et al. 1999). In contrast to this finding, a previous report demonstrated that dorsal, but not ventral, mPFC ibotenate lesions prevented the expression of cocaine-induced behavioral sensitization (Pierce et al. 1998). Taken together, these data suggest that lesions of regions of the ventral mPFC projecting to the VTA and shell of the NAc alter the initiation of sensitization, while lesions of dorsal part that provides dense projections to the core of the NAc disrupt the expression of sensitization (Pierce et al. 1998; Li et al. 1999; Li et al. 1999; Steketee 2005).

The anatomy and neurocircuitry within $\mathrm{mPFC}$ as described above make it in a position to modulate regions that have been previously linked to sensitization. In addition, changes in interactions between glutamate, dopamine and GABA in the $\mathrm{mPFC}$ have been hypothesized to play a critical role in the development of sensitization (Steketee 2003), thereby, indicating a potential role for these mPFC neurotransmitters in cocaine-induced sensitization. Therefore, from now on, the rest of this introduction will continue to review the role of the mPFC in the development of cocaine sensitization in terms of the major neurotransmitter systems within mPFC.

\section{Role of mPFC Dopaminergic System in Sensitization}

Some earlier reports demonstrated a reduced dopamine response to cocaine in the mPFC (Sorg et al. 1997; Chefer et al. 2000) rather than the enhanced response observed in other brain regions such as the NAc and VTA in cocaine sensitized animal (Kalivas and Duffy 1993). In contrast to these findings, a recent study found that sensitization is associated with enhanced dopamine transmission in the mPFC (Wu et al. 2003). However, a time course study from our lab demonstrated that when challenged 1 day or 1 week following repeated cocaine treatment, sensitized animals exhibited a decrease in cocaine-induced increases in dopamine overflow in the $\mathrm{mPFC}$ 
(Williams and Steketee 2005), while a sensitized mPFC dopamine response to cocaine was observed in sensitized animals after 30 days withdrawal (Williams and Steketee 2005). Generally, these data suggest that repeated cocaine produced time dependent alterations in $\mathrm{mPFC}$ dopamine transmission. The reduced dopamine response to cocaine in sensitized animals after a relatively short term withdrawal was suggested to result from a reduction in dopamine releasability rather than an increase in reuptake (Williams and Steketee 2004, 2005). The early reduced dopamine transmission in $\mathrm{mPFC}$ may contribute to the enhanced glutamate overflow in $\mathrm{mPFC}$ of cocaine sensitized animals (Williams and Steketee 2004).

The effects of mesocortical dopamine released in $\mathrm{mPFC}$ are produced by stimulating dopamine receptors including two families of receptors, $\mathrm{D}_{1 \text {-like }}$ and $\mathrm{D}_{2 \text {-like, }}$, which are both $G$ protein coupled receptors (Jackson and Westlind-Danielsson 1994). It has been suggested that both $\mathrm{D}_{1 \text {-like }}$ and $\mathrm{D}_{2 \text {-like }}$ receptors are located primarily on pyramidal glutamatergic neurons and GABAergic interneurons (al-Tikriti et al. 1992). $\mathrm{D}_{1 \text {-like }}$ dopamine receptors include $\mathrm{D}_{1}$ and $\mathrm{D}_{5}$ subtypes. Stimulation of these receptors can increase cAMP formation via Gs/o proteins (Dearry et al. 1990). In addition, these receptors can modulate intracellular calcium levels by stimulation of phospholipase $\mathrm{C}$ (Undie and Friedman 1990), and can increase L-type calcium channel current via PKA dependent phosphorylation pathways (Schiffmann et al. 1995; Surmeier et al.

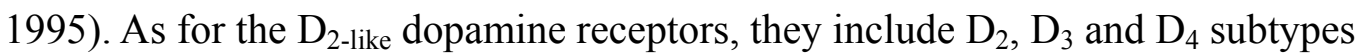
(Jackson and Westlind-Danielsson 1994; Missale et al. 1998). $\mathrm{D}_{2 \text {-like }}$ dopamine receptors are coupled to inhibitory Gi proteins, so as to inhibit the cAMP formation (Tang et al. 1994; McAllister et al. 1995). Additionally, $\mathrm{D}_{2 \text {-like }}$ dopamine receptors can inhibit the inward calcium current (Valentijn et al. 1993) and are indicated to be linked to inositol triphosphate production thereby modulating the intracellular calcium (Vallar et al. 1990) It should be noted that $\mathrm{D}_{2 \text {-like }}$ receptors can also be autoreceptors, which may modulate the release, synthesis and/or neuronal firing of the presynaptic neurons (Roth 1984). It should be noted that a recent finding showed that cocaine induced extracellular dopamine can suppress the activity of inwardly rectifying potassium channels in mPFC pyramidal neurons via $\mathrm{D}_{1}$ or $\mathrm{D}_{2}$ receptor dependent pathways so as to enhance the pyramidal neuron excitability (Dong et al. 2004). Generally, modulation of pyramidal neuron activity by dopamine receptors is very complex. There are concentration and receptor subtype dependent actions of dopamine in the PFC. Dopamine at low concentrations appears to act via $\mathrm{D}_{1}$ receptors

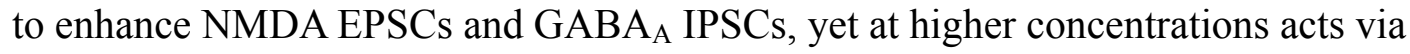
$\mathrm{D}_{2}$ receptors to reduce these currents (Trantham-Davidson et al. 2004) A recent review (Seamans and Yang 2004) discussed regulation of PFC pyramidal neuron by dopamine. The mechanisms of regulation were summed up as follows: Activation of $\mathrm{D}_{1}$ and $\mathrm{D}_{2}$ receptors inhibits spontaneous firing of $\mathrm{PFC}$ neurons. Activation of $\mathrm{D}_{1}$ receptor stimulates interneurons, thereby inhibiting pyramidal neuron activity. When strong depolarization removes the voltage-dependent $\mathrm{Mg}^{2+}$ block of NMDA channels, activation of $D_{1}$ receptor enhances the $D_{1}$-mediated enhancement of NMDA receptors by enhancing persistent $\mathrm{Na}^{+}$and inactivating $\mathrm{K}^{+}$current through a PKA 
phosphorylation-independent process. Thus, dopamine is neither a classic excitatory nor inhibitory neurotransmitter (Lapish et al. 2007). Furthermore, activation of $\mathrm{D}_{2}$ receptors, located on interneurons, stimulates GABA release (Grobin and Deutch 1998) that can then serve to inhibit glutamatergic neurons (Pirot et al. 1992).

Therefore, dopamine is capable of directly and indirectly inhibiting pyramidal output neurons.

Dopamine receptors in the $\mathrm{mPFC}$ have been implicated in the development of cocaine induced sensitization. It has been shown that intra-ventral $\mathrm{mPFC}$ infusion of $D_{1}$ receptors agonist SKF 81297 did not block the initiation of cocaine sensitization (Beyer and Steketee 2002). But some study indicated that $\mathrm{D}_{1}$ receptors in dorsal regions of the $\mathrm{mPFC}$ may be involved in expression of cocaine sensitization (Sorg et al. 2001). Also infusions of the dopamine $\mathrm{D}_{2}$ agonist quinpirole into the $\mathrm{mPFC}$ blocked the initiation of sensitization to cocaine (Beyer and Steketee 2002). Once sensitization was induced, quinpirole was only able to attenuate, rather than completely block the motor stimulant response to cocaine (Beyer and Steketee 2002). Repeated intra-mPFC injection of the $\mathrm{D}_{2}$ antagonist sulpiride even induced sensitization to cocaine. (Steketee and Walsh 2005) Taken together, the results of these studies suggest that sensitization may result from attenuated dopamine $\mathrm{D}_{2}$ receptor function in the ventral mPFC. Also, a recent study demonstrated that quinpirole induced GTP $\gamma \mathrm{S}$ binding was reduced in the $\mathrm{mPFC}$ of sensitized animals (Bowers et al. 2004). These studies suggested that a decrease in $m P F C D_{2}$ function can enhance excitatory transmission to subcortical regions. And the reduction in $\mathrm{mPFC}_{2}$ function is correlated with long term expression of cocaine induced behavioral sensitization (Beyer and Steketee 2002; Bowers et al. 2004).

\section{Role of mPFC GABAergic System in Sensitization}

As described above, mPFC GABA is released from mPFC interneurons and terminals of VTA projections. There are two receptor subtypes in the mPFC: GABA and $\mathrm{GABA}_{\mathrm{B}}$. $\mathrm{GABA}_{\mathrm{A}}$ receptors are ionotropic receptors that gate chloride while $\mathrm{GABA}_{\mathrm{B}}$ receptors are metabotropic receptors coupled to $\mathrm{Ca}^{2+}$ and $\mathrm{K}^{+}$channels by $\mathrm{Gi}$ proteins (Nutt 2006; Olsen et al. 2007). It has been demonstrated that stress induced increases in NAc dopamine overflow was blocked by intra-mPFC injections of the $\mathrm{GABA}_{\mathrm{B}}$ agonist baclofen (Doherty and Gratton 1999). Sensitization to amphetamine was associated with an increase in $\mathrm{GABA}_{\mathrm{B}}$ receptor coupling to $\mathrm{G}$ proteins but not $\mathrm{GABA}_{\mathrm{B}}$ receptor number in mPFC (Zhang et al. 2000; Kushner and Unterwald 2001). In contrast to this, repeated exposure to cocaine was not found to alter $G_{A B A}$ receptor coupling in the frontal cortex (Kushner and Unterwald 2001). Intra-mPFC injection of baclofen blocked the acute motor-stimulant response to cocaine in a dose-dependent manner. When paired with the daily injections of systemic cocaine, intra-mPFC baclofen blocked the initiation of sensitization. However, once sensitization was induced, intra-mPFC baclofen was not able to block the expression 
of sensitization (Steketee and Beyer 2005). These data suggest that $\mathrm{GABA}_{\mathrm{B}}$ receptor function is lost following repeated cocaine exposure. Furthermore, infusions of baclofen into the mPFC generated trends towards decreased glutamate levels in the mPFC, nucleus accumbens and VTA in control animals, while increasing levels of glutamate in each of these same regions in cocaine-sensitized animals (Jayaram and Steketee 2004). Since $\mathrm{GABA}_{B}$ autoreceptor function appears to be intact because baclofen reduces GABA to a similar extent in both control and sensitized animals (Jayaram and Steketee 2004), it is hypothesized that postsynaptic/heterosynaptic $\mathrm{GABA}_{\mathrm{B}}$ receptor function is lost following repeated cocaine, thus $\mathrm{mPFC}$ infusions of baclofen removed postsynaptic and heterosynaptic inhibitory tone produced by the action of GABA on $\mathrm{GABA}_{\mathrm{A}}$ receptors, thereby increasing excitatory transmission in the mesocorticolimbic system (Jayaram and Steketee 2004; Steketee and Beyer 2005). Although the mechanisms by which repeated cocaine reduced $\mathrm{GABA}_{\mathrm{B}}$ receptor function are not clear, it is possible that decreased $\mathrm{GABA}_{\mathrm{B}}$ receptor function is a compensatory response to increased GABA transmission. Our lab reported that acute cocaine did not affect the GABA levels in $\mathrm{MPFC}$, but a challenge injection of cocaine in sensitized animals induced an increase in mPFC GABA levels after 1 or 7 days withdrawal but not 30 days withdrawal from repeated cocaine exposure (Jayaram and Steketee 2005). While it is not clear how repeated cocaine alters mPFC GABA levels, recent studies suggest that the transient increase in $\mathrm{MPFC}$ GABA response to cocaine may be due to the enhanced glutamate transmission via AMPA receptors associated with cocaine sensitization (Jayaram and Steketee 2006).

\section{Role of mPFC Glutamatergic System in Cocaine}

Numerous studies have demonstrated an important role for mPFC glutamate systems in the development of sensitization (Wolf 1998; Kalivas 2004; Steketee 2005). Glutamate neurons and terminals located in the mPFC provide the excitatory drive for pyramidal output that project to many subcortical regions. Several recent reports suggested that cocaine mediated behaviors can also be modulated, in part, by excitatory transmission from the $\mathrm{mPFC}$ to subcortical dopaminergic regions (Wolf 1998; Tzschentke 2000; Steketee 2003). Since the pyramidal neurons are the major output neurons in $\mathrm{mPFC}$, modulation of pyramidal neurons could potentially be involved in regulation of cocaine mediated behaviors. Acute cocaine has been reported to increase extracellular glutamate levels in the mPFC (Reid et al. 1997). Repeated cocaine administration increases membrane excitability of pyramidal neurons in the rat mPFC via a reduction in voltage-gated $\mathrm{K}^{+}$currents (VGKCs) and a possible increase in voltage-sensitive $\mathrm{Ca}^{2+}$ currents (Nasif et al. 2005; Nasif et al. 2005). Consistent with this study, evidence has suggested that glutamate transmission in the mPFC may be altered in sensitized animals. Neurons in the mPFC show an increased responsiveness to glutamate following repeated exposure to amphetamine (Peterson et al. 2000). $\mathrm{K}^{+}$-stimulated glutamate efflux in the mPFC was enhanced in animals following repeated methamphetamine exposure (Stephans and Yamamoto 
1995). Time courses study of the effects of repeated cocaine exposure on subsequent $\mathrm{mPFC}$ glutamate responses to cocaine from our lab has shown time-dependent (1 day or 7 days but not 30 days withdrawal) increases in cocaine mediated mPFC glutamate overflow after repeated cocaine exposure, although an acute injection of cocaine failed to increase mPFC extracellular glutamate levels (Williams and Steketee 2004). Therefore, alterations in mPFC glutamate neurotransmission may be involved in the development of sensitization.

As discussed earlier, sensitization is believed to result from an increase in excitatory transmission from the $\mathrm{mPFC}$ to subcortical regions. Enhanced excitatory outputs from the $\mathrm{mPFC}$ can result from either an increase in excitatory drive or a decrease in inhibitory regulation of pyramidal neurons (Steketee 2005). As described above, a reduction in $\mathrm{D}_{2}$ and $\mathrm{GABA}_{\mathrm{B}}$ receptor function could produce a decrease in inhibition of glutamate transmission in $\mathrm{mPFC}$. Glutamate can regulate pyramidal neuron activation by stimulation of both ionotropic and metabotropic glutamate receptors. Ionotropic glutamate receptors basically are glutamate gated cation channels, which are permeable to $\mathrm{Ca}^{2+}, \mathrm{Na}^{+}$and $\mathrm{K}^{+}$(Dingledine et al. 1999). Generally, activation of ionotropic glutamate receptors enhances the neuronal excitability. Moreover, it has been reported that ionotropic glutamate receptors, including NMDA, AMPA and kainate receptors are located on both pyramidal neurons and GABA interneurons within mPFC (Huntley et al. 1994).

In addition to ionotropic glutamate receptors, metabotropic glutamate receptors (mGluRs) are also important. Currently, there are eight subtypes of mGluRs (shown in Table 1-1), classified into three groups of receptors based on sequence homology, preferred signal transduction pathway and pharmacology (Conn and Pin 1997; Kenny and Markou 2004). Group I ( $\left.\mathrm{mGluR}_{1 / 5}\right)$ can increase the cleavage of phosphoinositidyl inositol biphosphate (PIP2) by activation of PLC. Group II $\left(\mathrm{mGluR}_{2 / 3}\right)$ and Group III (mGluR $\left.\mathrm{m}_{4 / 6 / 7 / 8}\right)$ both can inhibit the adenylyl cyclase-mediated formation of cAMP (Cartmell and Schoepp 2000). Within the cortex, there are many subtypes of mGluRs, however, the Group I and II receptors are abundant in the mPFC area (Watkins 2000). As for the specific localization of these receptors, Group I receptors tend to be located postsynaptically on both pyramidal neurons and GABA interneurons within mPFC (Muly et al. 2003). Group II mGluRs were indicated to play an inhibitory role in regulating glutamate release. (Conn and Pin 1997) Group II receptors can also be localized postsynaptically and on glia cells as inhibitory receptors (Petralia et al. 1996). Importantly, Group II receptors can also function as presynaptic heteroceptors on monoaminergic terminals in the $\mathrm{mPFC}$ or as autoreceptors inhibiting presynaptic glutamate release (Petralia et al. 1996; Conn and Pin 1997; Xi et al. 2002). $\mathrm{mGluR}_{2}$ and $\mathrm{mGluR}_{3}$ are found in various combinations of presynaptic, postsynaptic and glial localizations that may reflect differential modulation of excitatory amino acid transmission (Petralia et al. 1996; Conn and Pin 1997). Postsynaptic group II mGluRs in rat prefrontal cortex can induce LTD through postsynaptic PKC activation and IP3 receptor-mediated postsynaptic increases of $\mathrm{Ca}^{2+}$ 
Table 1-1. Classification of metabotropic glutamate receptor subtypes.

\begin{tabular}{lll}
\hline & Subtype & Receptor Coupled G Proteins \\
\hline \multirow{2}{*}{ Group I } & & $\mathrm{Gq}$ \\
& $\mathrm{mGluR}_{1}$ & $\mathrm{Gq}$ \\
$\mathrm{mGluR}_{5}$ & $\mathrm{Gi} / \mathrm{o}$ \\
Group II & $\mathrm{mGluR}_{2}$ & $\mathrm{Gi} / \mathrm{o}$ \\
& $\mathrm{mGluR}_{3}$ & \\
Group III & $\mathrm{mGluR}_{4}$ & $\mathrm{Gi} / \mathrm{o}$ \\
& $\mathrm{mGluR}_{6}$ & $\mathrm{Gi} / \mathrm{o}$ \\
& $\mathrm{mGluR}_{7}$ & $\mathrm{Gi} / \mathrm{o}$ \\
& $\mathrm{mGluR}_{8}$ & $\mathrm{Gi} / \mathrm{o}$ \\
& & \\
\hline
\end{tabular}


concentration via phospholipase $\mathrm{C}$ and probably also phospholipase $\mathrm{D}$ (Otani et al. 2002). LY314582, an agonist at inhibitory Group II mGluRs, was recently shown to elevate ICSS thresholds (Kenny et al. 2003). This observation suggests that Group II mGluRs negatively regulate brain reward function. Blockade of Group II mGluRs in the NAc produced hyperlocomotion in rats previously exposed to amphetamine (Chi et al. 2006). A recent study using $\mathrm{mGluR}_{2} /-$ knockout (KO) mice was conducted to explore the physiological role of $\mathrm{mGluR}_{2}$ in brain function. Although these knockout mice appeared to have no behavioral abnormalities, they showed a significant increase in locomotor sensitization and conditioned place preference in association with repeated cocaine administration (Morishima et al. 2005). This evidence prompted us to design studies to examine the role of Group II mGluRs in the development of cocaine sensitization in support of our hypothesis that repeated exposure to cocaine changes inhibitory modulation of pyramidal neurons, leading to increased excitatory outputs to other subcortical brain regions including VTA and NAc, which are associated with the development of cocaine sensitization (Steketee 2003). Reduction in Group II mGluRs function within either NAc or mPFC has also been suggested to be associated with the long term expression of cocaine sensitization, although few studies have demonstrated the potential role of Group II mGluRs of mPFC in the development of sensitization (Xi et al. 2002; Bowers et al. 2004).

\section{Summary}

Although the long term neuroadaptive effects of psychomotor stimulants on the brain have been studied for many years, much is still left to learn. Behavioral sensitization as a classic animal model has been well used in investigating these neuronal changes in the brain. Behavioral sensitization is referred to the augmented locomotor response to drug challenge after repeated drug exposure. Many studies including those from our lab have shown the importance of medial prefrontal cortex (mPFC) in the development of cocaine induced behavioral sensitization, which is thought to play an important role in craving and relapse in drug addiction (Steketee 2003; Kalivas 2004). The mPFC is the major source of excitatory output to the VTA and NAc, regions previously linked to sensitization (Wolf 1998; Kalivas 2004; Steketee 2005). Recent studies in our lab suggest that cocaine-induced sensitization results from a reduction in inhibitory modulation of these pyramidal neurons, which possibly results from the reduced function of several $\mathrm{G}$ protein coupled receptors in $\mathrm{mPFC}$, including $\mathrm{D}_{2}, \mathrm{GABA}_{\mathrm{B}}$ as well as $\mathrm{mGluR}_{2 / 3}$ receptors, acting as both autoreceptors and inhibitory postsynaptic receptors (Steketee 2005). Therefore, further understanding of the modulation of glutamate output during the development of sensitization may not only help to further understand the mechanism of sensitization but also provide new pharmacotherapy for drug addiction. 


\section{Specific Aims}

Previous studies have implicated an important role for $\mathrm{mGluR}_{2 / 3}$ receptors in the NAc in cocaine mediated responses (Xi et al. 2002; Peters and Kalivas 2006). Similar support has yet to be presented for $\mathrm{mGluR}_{2 / 3}$ receptors in the $\mathrm{mPFC}$, although a study has indicated reduced $\mathrm{mGluR}_{2 / 3}$ receptor function in $\mathrm{mPFC}$ after repeated cocaine exposure (Bowers et al. 2004). Therefore it is expected that this reduced inhibitory transmission in the $\mathrm{mPFC}$ may contribute to the increased excitatory output from glutamate pyramidal neurons to NAc and VTA seen in cocaine-sensitized animals. The increased excitatory output will increase mesolimbic dopamine neuron activity and enhance dopamine release in NAc, previously thought to be a critical brain area for expression of sensitization. Furthermore, repeated cocaine exposure may reduce the inhibitory tone on the mPFC glutamate pyramidal cells so as to change neurotransmission, since infusion of an $\mathrm{mGluR}_{2 / 3}$ receptor antagonist increased $\mathrm{mPFC}$ glutamate levels, but not an agonist (Melendez and Kalivas 2003), indicating that $\mathrm{mGluR}_{2 / 3}$ has an inhibitory tone on the $\mathrm{mPFC}$ glutamate levels. Thus, it is necessary to examine whether activation of $\mathrm{mPFC} \mathrm{mGluR}_{2 / 3}$ receptors can prevent the initiation of behavioral sensitization, and whether the effect of $m P F C \mathrm{mGluR}_{2 / 3}$ receptors on regulating behavioral sensitization will be reduced following repeated exposure to cocaine and also demonstrate how the alteration of glutamate neurotransmission within mesocorticolimbic brain regions by $\mathrm{mGluR}_{2 / 3}$ receptor is correlated with behavioral responses induced by repeated cocaine exposure.

Studies have demonstrated a transient cocaine induced increase in mPFC glutamate overflow in sensitized animals (Williams and Steketee 2004), however the source of the glutamate was not determined. Glutamate can be released from both mPFC afferent terminals and axon collaterals of pyramidal neurons. $\mathrm{K}^{+}$-stimulated glutamate efflux in the mPFC was enhanced in animals following repeated methamphetamine exposure (Stephans and Yamamoto 1995). However, it has yet to be known whether repeated cocaine exposure can alter this $\mathrm{K}^{+}$evoked glutamate release in the $\mathrm{mPFC}$ and whether a reduction of $\mathrm{mGluR}_{2 / 3}$ receptor function may be one of the possible mechanisms involved in the alteration of glutamate release from this vesicular pool in cocaine sensitized animals. Moreover, there is at least one other glutamate pool. Recent studies in the nucleus accumbens suggest that cocaine influences a pool of glutamate regulated by the cystine/glutamate antiporter (Baker et al. 2002; Baker et al. 2002). This antiporter, which is located mainly on glia cells, is a $\mathrm{Na}^{+}$-dependent anionic amino acid antiporter that exchanges intracellular glutamate for extracellular cystine (Baker et al. 2002). In addition to acting as an autoreceptor, $\mathrm{mGluR}_{2 / 3}$ receptors can decrease extracellular glutamate levels by inhibiting the cystine/glutamate antiporter (Baker et al. 2003). Therefore, it is necessary to examine whether repeated cocaine exposure can alter release of glutamate from this pool in $\mathrm{mPFC}$ and whether the reduction of $\mathrm{mGluR}_{2 / 3}$ receptor function may also be one of the possible mechanisms involved in alterated glutamate release from this pool in cocaine sensitized animals. 
In summary, it is hypothesized that repeated exposure to cocaine changes neurotransmitter-mediated modulation of pyramidal neurons, leading to increased excitatory output to the VTA and NAc. The specific aims of this project will examine the role for $\mathrm{mPFC} \mathrm{mGluR}_{2 / 3}$ in the development of cocaine sensitization and determine how repeated cocaine exposure alters mesocorticolimbic glutamate transmission via alterations of $\mathrm{mGluR}_{2 / 3}$ receptor function. This project will also determine the pools of mPFC glutamate affected by cocaine, as well as the potential mechanisms by which glutamate transmission is altered. The following is the description of the specific aims of this dissertation research project, which were examined in next two chapters.

\title{
$\operatorname{Aim} 1$
}

Aim 1 is to study the role of $\mathrm{mGluR}_{2 / 3}$ in the development of behavioral and neurochemical sensitization. Aim 1 will determine whether intra-mPFC injection of the mGluR $_{2 / 3}$ agonist 2R, 4R-4-aminopyrrolidine-2, 4-dicarboxylate (APDC) prevents the initiation and/or expression of the development of cocaine sensitization.

\begin{abstract}
$\operatorname{Aim} 2$
Aim 2 is to study effects of repeated cocaine exposure on mPFC mGluR $2 / 3$ receptor function. Studies will determine whether repeated cocaine exposure decreases intra-mPFC APDC modulation of mesocorticolimbic glutamate levels.
\end{abstract}

\begin{abstract}
$\operatorname{Aim} 3$
Aim 3 is to study the releasable pools of glutamate within mPFC affected by cocaine and examine the possible mechanisms underlying the alteration by $\mathrm{mPFC}$ $\mathrm{mGluR}_{2 / 3}$ receptor. The effects of intra-mPFC APDC infusion on cystine and/or $\mathrm{K}^{+}$ induced glutamate release in control and sensitized animals will be determined.
\end{abstract}




\section{Chapter 2. Repeated Exposure to Cocaine Reduces the Ability of Medial Prefrontal Cortical Group II Metabotropic Glutamate Receptors to Modulate Behavioral and Neurochemical Responses to Cocaine}

\section{Introduction}

Repeated administration of psychostimulants such as cocaine can generate a progressive enhancement in locomotor activity that can last for months to years. This phenomenon is termed behavioral sensitization (Kalivas and Duffy 1990; Kalivas et al. 1993; Steketee 2003). The gradual neuroadaptations that render the animals hypersensitive to psychostimulants are thought to underlie relapse and compulsive drug-seeking and drug-taking behaviors (Robinson and Berridge 1993; Kalivas et al. 1998). Therefore, sensitization is used as a model to study drug addiction. It has been widely suggested that alterations of dopamine (DA) transmission in the ventral tegmental area (VTA) and nucleus accumbens (NAc) are responsible for the initiation and expression, respectively, of behavioral sensitization induced by psychostimulants (Kalivas et al. 1993; Robinson and Berridge 1993; Vanderschuren and Kalivas 2000; Cornish and Kalivas 2001; Carlezon and Nestler 2002). However, recent studies have suggested that additional brain regions such as the medial prefrontal cortex are also involved in the development of sensitization (Steketee 2005).

The medial prefrontal cortex ( $\mathrm{mPFC}$ ), which is a component of the mesocorticolimbic dopamine system, contains glutamatergic pyramidal neurons, the major output neurons, which innervate numerous brain regions, including the nucleus accumbens (NAc) and ventral tegmental area (VTA) (Sesack et al. 1989; Takagishi and Chiba 1991; Pierce and Kalivas 1997). Lesion studies initially implicated a critical role of the mPFC in the development of cocaine-induced sensitization. For instance, bilateral lesions of the $\mathrm{mPFC}$ with ibotenic acid before repeated cocaine treatment prevented the induction of cocaine sensitization and the associated neuroadaptations ( $\mathrm{Li}$ et al. 1999). Glutamate transmission from the mPFC has been shown to be increased in sensitized animals (Wolf 1998; Steketee 2003; Kalivas 2004). The increased excitatory $\mathrm{mPFC}$ output may at least partially result from a reduction in inhibitory modulation of mPFC pyramidal neurons. For instance, it has been demonstrated that infusions of the dopamine $\mathrm{D}_{2}$ agonist quinpirole or the $\mathrm{GABA}_{\mathrm{B}}$ agonist baclofen into the $\mathrm{mPFC}$ blocked the initiation of sensitization to cocaine. However, once sensitization was induced, intra-mPFC quinpirole was only able to attenuate, while baclofen failed to alter the expression of sensitization (Beyer and Steketee 2002; Steketee and Beyer 2005).

There are eight mGluRs, which are classified into three groups of receptors based on sequence homology, preferred signal transduction pathway and pharmacology (Vezina and Kim 1999; Gerber et al. 2007). Immunohistochemical studies have 
revealed the distribution of $\mathrm{mGluR}_{2 / 3}$ receptors. The neocortex and various limbic cortical regions contain high receptor levels (Conn and Pin 1997). $\mathrm{mGluR}_{2 / 3}$ is a Gi protein coupled receptor that inhibits cAMP formation. GluR $_{2 / 3}$ receptor can act as both postsynaptic inhibitory receptor and autoreceptors. $\operatorname{mGluR}_{2 / 3}$ receptors are found in various combinations of presynaptic, postsynaptic and glial localizations that reflect differential modulation of excitatory amino acid transmission (Cartmell and Schoepp 2000; Kenny and Markou 2004). Previous studies have indicated that Group II metabotropic glutamate receptors (mGluRs), which include $\mathrm{mGluR}_{2}$ and $\mathrm{mGluR}_{3}$ $\left(\mathrm{mGluR}_{2 / 3}\right)$ can also play an inhibitory role in regulating $\mathrm{mPFC}$ glutamate release. For instance, mGluR $_{2 / 3}$ agonist $2 \mathrm{R}, 4 \mathrm{R}$-4-aminopyrrolidine-2,4-dicarboxylate (APDC) can activate $\mathrm{K}^{+}$currents to reduce the presynaptic action potential amplitude, which is ideal for the decrement of synaptic transmission. (Cartmell and Schoepp 2000) The selective mGluR $_{2 / 3}$ agonist (2S,2'R,3'R)-2-(2',3'-dicarboxycyclopropyl)glycine (DCG-IV) reduced excitatory synaptic transmission in the mPFC (Otani et al. 2002). Based on the information described above, it is expected that like $\mathrm{GABA}_{\mathrm{B}}$ and dopamine $\mathrm{D}_{2}$ receptors, $\mathrm{mPFC} \mathrm{mGluR}_{2 / 3}$ receptors could modulate behavioral responses to cocaine. While this has yet to be explored, previous studies showed that both systemic and intra-NAc core pretreatment with (1R,4R,5S,6R)-4-Amino-2-oxabicyclo[3.1.0]hexane-4,6-dic arboxylic acid (LY379268), a mGluR $2 / 3$ agonist, inhibited cocaine seeking behavior (Peters and Kalivas 2006). Pretreatment with systemic LY379268 reduced cocaine self-administration and cocaine-induced reinstatement of drug seeking behavior in a dose-dependent manner, an affect that could be reversed by (2S)-2-Amino-2-[(1S,2S)-2-carboxycycloprop-1-yl]-3-(xan th-9-yl) propanoic acid (LY341495), an GluR $_{2 / 3}$ antagonist (Adewale et al. 2006). However, the potential role of $\mathrm{mPFC} \mathrm{mGluR}_{2 / 3}$ receptors in mediating drug related behavior remains to be examined.

Based on the discussion above, the studies described below were designed to test the hypothesis that the ability of activation of $\mathrm{mPFC} \mathrm{mGluR}_{2 / 3}$ receptors to reduce behavioral and neurochemical responses to cocaine is lost in cocaine sensitized animals. The present studies will test this hypothesis by examining the effects of intra-mPFC injection of $\mathrm{mGluR}_{2 / 3}$ receptor agonist, APDC on the acute and sensitized locomotor and dopamine responses to cocaine in the NAc.

\section{Materials and Methods}

\section{Animals and Surgery}

Male Spraque-Dawley rats that weighed 275-300 $\mathrm{g}$ at the time of surgery, were housed under $12 \mathrm{hr}$ light/dark cycle and had free access to food and water. Rats were housed in groups of four before surgery and were individually housed after surgery. Prior to surgery, rats were anaesthetized with Equithesin $(3.3 \mathrm{ml} / \mathrm{kg})$ and their heads 
were mounted in a stereotaxic frame (Kopf Instruments). The scalp was incised to expose the skull, and bregma and lambda were aligned in the same horizontal plane. Rats received bilateral implants of 20 gauge $(14 \mathrm{~mm})$ microdialysis guide cannulae $3.0 \mathrm{~mm}$ above the NAc $(+1.4 \mathrm{~mm}$ posterior to bregma and $+1.4 \mathrm{~mm}$ lateral to the midline, and $-5.0 \mathrm{~mm}$ ventral from dura), and/or 26 gauge $(14 \mathrm{~mm})$ microinjection guide cannulae $1.0 \mathrm{~mm}$ above the $\mathrm{mPFC}(+3.2 \mathrm{~mm}$ posterior to bregma and $+0.6 \mathrm{~mm}$ lateral to the midline, and $-3.5 \mathrm{~mm}$ ventral from dura). (Paxinos and Watson, 1997) Cannulae were anchored with 3 stainless steel screws and dental acrylic. Obturators ( 25 or 33 gauge, $14 \mathrm{~mm}$ ) were inserted into the cannulae in order to prevent their occlusion. Animals were allowed at least one week for recovery from surgery. All procedures were conducted in accordance with the National Institutes of Health Guide for the Care and Use of Laboratory Animals. The University of Tennessee Health Science Center Animal Resources Advisory Committee approved all experimental procedures.

\section{Microinjections}

The rats were manually restrained and obturators were removed from the guide cannulae. Stainless steel injectors ( $15 \mathrm{~mm}, 33$ gauge) attached to $1 \mu 1$ syringes via PE 20 tubing mounted on a Sage syringe pump were then inserted into the guide cannulae. The tip of the injectors protruded $1 \mathrm{~mm}$ beyond the tip of the guide cannulae into the $\mathrm{mPFC}$. Animals were not restrained during injection. The infusion rate was $0.5 \mu \mathrm{l} / \mathrm{min}$ and a total of $0.5 \mu \mathrm{l}$ was injected on each side. After infusion, injectors were left in place for 30 seconds to allow for diffusion of the infused solution and obturators were reinserted into guide cannulae after injections. If animals were receiving microinjections as a pretreatment, they were typically given $5 \mathrm{~min}$ before systemic injections. In acute cocaine response studies, animals were injected a maximum of 4 times with a minimum 3-day interval.

\section{Behavior}

A Digiscan Micro Monitoring system connected to a personal computer was used to measure motor activity. This system consists of individual activity boxes resting on sensor-placed aluminum frames. The frames are equipped with 16 light beams and detectors, which are connected through an analyzer to the computer. Photocell counts are recorded when the animal breaks the individual beams. For acute cocaine dose response studies, animals were adapted to the activity boxes for $60 \mathrm{~min}$ before injection. Motor activity was monitored for $2 \mathrm{~h}$ with $15 \mathrm{~min}$ intervals following injection. Separate groups of animals were used to test four different doses of APDC and each group of animals received each of four possible treatment combinations (saline/saline, saline/cocaine, APDC/saline, and APDC/cocaine) in a random order with a minimum 3-day intertrial interval. For APDC and LY341495 co-injection studies, animals were exposed to four of eight possible treatment combinations 
(saline/saline, saline/cocaine, APDC/saline, APDC/cocaine, LY341495/saline, LY341495/cocaine, APDC+LY341495/saline and APDC+LY341495/cocaine) in a random order with a minimum 3-day intertrial interval. Treatment procedures that determined the effects of intra-mPFC APDC on the initiation and expression of sensitization to cocaine are shown in Table 2-1.

\section{In vivo Microdialysis}

Microdialysis experiments were conducted with conscious, freely moving rats. On day 1 of the microdialysis studies the animals underwent 'sham dialysis' in which they were given microinjections in the chambers but were not dialyzed. For the next 3 days, animals received the same treatment in their home cages. Microdialysis probes with a membrane length of $2 \mathrm{~mm}$ were inserted into guide cannulae the night before (at least $16 \mathrm{hr}$ ) conduction of the experiment in order to minimize damage-induced release of neurotransmitters and metabolites. The probe was continuously perfused with dialysis buffer $\left(\mathrm{KCl} 2.7 \mathrm{mM}, \mathrm{NaCl} 140 \mathrm{mM}, \mathrm{CaCl}_{2} 1.2 \mathrm{mM}, \mathrm{MgCl}_{2} 1.2 \mathrm{mM}\right.$, plus $0.2 \mathrm{mMphosphate-buffered} \mathrm{saline} \mathrm{to} \mathrm{achieve} \mathrm{a} \mathrm{pH}=7.4$ ) at a flow rate of $2 \mu \mathrm{l} / \mathrm{min}$ during sample collection. The flow rate was reduced to $0.003 \mu 1 / \mathrm{min}$ during the overnight period and was adjusted back to $2 \mu \mathrm{l} / \mathrm{min}$ at least $1 \mathrm{hr}$ before the beginning of sample collection. Four $20 \mathrm{~min}$ baseline samples were collected before animals received intracranial and systemic injections. Nine 20 min samples were collected after injections. Motor activity data were collected in conjunction with dialysis sample collection as described above. Samples were collected into $20 \mu \mathrm{l}$ of $0.05 \mathrm{mM} \mathrm{HCl}$ in $0.5 \mathrm{ml}$ microcentrifuge tubes. Samples either immediately underwent high performance liquid chromatography (HPLC) analysis of dopamine or were stored at $-80^{\circ} \mathrm{C}$. Samples were stored no more than 2 weeks (Beyer and Steketee 2002).

\section{High Performance Liquid Chromatography}

The dopamine HPLC system consists of an octadecasilane reversed-phase column ( $2 \mathrm{~mm}$ x $15 \mathrm{~cm}$ narrowbore, $3 \mu \mathrm{m}$, ESA Inc., Chelmsford, MA), an ESA Model 582 solvent delivery module, an ESA Model 542 refrigerated autosampler and an electrochemical system including a guard cell $(+400 \mathrm{mV})$ and an amperometric cell $(+150 \mathrm{mV})$. Mobile phase $\left(50 \mathrm{mM} \mathrm{NaH}_{2} \mathrm{PO}_{4}, 2 \mathrm{mM}\right.$ decanesulfonic acid, $0.7 \mathrm{mM}$ EDTA, $11 \%$ acetonitrile $\mathrm{v} / \mathrm{v}$ and $11 \%$ methanol $\mathrm{v} / \mathrm{v}, \mathrm{pH}=6.0$ with $6 \mathrm{~N} \mathrm{NaOH}$ ) was pumped at a rate of $0.25 \mathrm{ml} / \mathrm{min}$. Dopamine detected by the electrochemical system was compared to an external standard curve (1-300 fmol) for quantification.

\section{Histology}

After completion of studies that involve intracranial cannula implantation, animals were deeply anesthetized with sodium pentobarbital and were perfused by 
Table 2-1. Treatment regimen for sensitization to cocaine.

Initiation of cocaine sensitization

$\begin{array}{llllll}\text { Day } & 1 & 2 & 3 & 4 & 11\end{array}$

$\begin{array}{llllll}\text { Saline/Saline } & \mathrm{s} / \mathrm{s} & \mathrm{s} / \mathrm{s} & \mathrm{s} / \mathrm{s} & \mathrm{s} / \mathrm{s} & \mathrm{C} \\ \text { Saline/Cocaine } & \mathrm{s} / \mathrm{c} & \mathrm{s} / \mathrm{c} & \mathrm{s} / \mathrm{c} & \mathrm{s} / \mathrm{c} & \mathrm{C} \\ \text { APDC/Saline } & \mathrm{a} / \mathrm{s} & \mathrm{a} / \mathrm{s} & \mathrm{a} / \mathrm{s} & \mathrm{a} / \mathrm{s} & \mathrm{C} \\ \text { APDC/Cocaine } & \mathrm{a} / \mathrm{c} & \mathrm{a} / \mathrm{c} & \mathrm{a} / \mathrm{c} & \mathrm{a} / \mathrm{c} & \mathrm{C}\end{array}$

Expression of cocaine sensitization

$\begin{array}{llllll}\text { Day } & 1 & 2 & 3 & 4 & 5,11,34\end{array}$

\begin{tabular}{llllll} 
Saline/Saline & s & s & s & s & S/C \\
Saline/Cocaine & c & c & c & c & S/C \\
APDC/Saline & s & s & s & s & A/C \\
APDC/Cocaine & c & c & c & c & A/C \\
\hline
\end{tabular}

$\mathrm{s}=$ saline, $\mathrm{a}=\mathrm{APDC}$ (APDC injected into the $\mathrm{mPFC}$ ) and $\mathrm{c}=$ cocaine $(15 \mathrm{mg} / \mathrm{kg}$, ip). Uppercase letters represent days when motor activity data were collected following injection and lowercase letters represent days when animals received injections in their home cages. 
intracardiac infusion of phosphate-buffered saline $(50 \mathrm{ml})$ and $10 \%$ formaldehyde $(50$ $\mathrm{ml}$ ). Brains were stored in vials with $10 \%$ formaldehyde for at least 3 days before sectioning. Brains were sectioned $(100 \mu \mathrm{m})$ on a vibratome and sections were mounted onto gelatin-coated slides. Sections were stained with cresyl violet and injection sites and/or dialysis probe placements were visualized by light microscopy and verified according to Paxinos and Watson (1997).

\section{Drugs}

Cocaine hydrochloride was purchased from Sigma Chemical Company. (St Louis, MO) APDC and LY341495 were purchased from TOCRIS Bioscience. (Ellisville, MO) APDC was diluted with isotonic saline. (0.9\% sodium chloride) LY341495 was diluted with 1.2eq. $\mathrm{NaOH}$.

\section{Statistics}

A two-way analysis of variance (ANOVA) with one repeated measure (time) was used to analyze time courses of behavioral and neurochemical data. The microdialysis data were converted to percentage of baseline in order to avoid variability associated with differences in probe placement and probe recovery. Multiple comparisons were made with a modified least significant differences test (Milliken and Johnson 1984). For dose-response studies total activity data were analyzed by a one-way ANOVA with repeated measures. Multiple comparisons were made with a student Newman-Keuls test.

\section{Results}

\section{Histology}

Figure 2-1 shows typical injection sites and dialysis probe placements in the mPFC and NAc respectively. Generally, injections sites were located in the ventral portions of the $\mathrm{mPFC}$, specifically in the infralimbic and ventral prelimbic subregions. For the microdialysis studies, the tracks of dialysis probes were found to be at or medial to the anterior commissure and most of the probe was contained within the core and/or shell of the nucleus accumbens. Animals with incorrect injection sites or dialysis probe placements were removed from the study. 

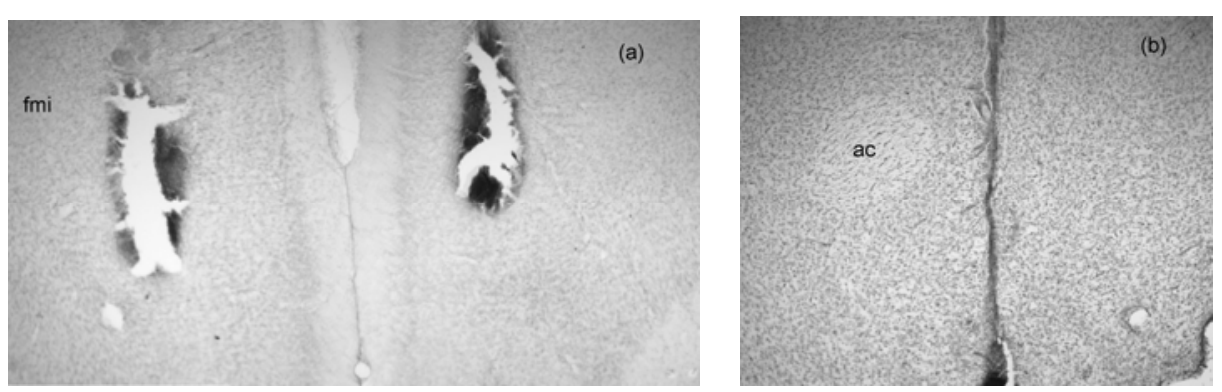

Figure 2-1. Injection sites and dialysis probe placements in the $\mathrm{MPFC}$ and NAc. Generally, injections sites were located in the ventral portions of the mPFC (a), specifically in the infralimbic and ventral prelimbic subregions. For the microdialysis studies, the tracks of dialysis probes were found to be at or medial to the anterior commissure and most of the probe was contained within the core and/or shell of the NAc (b). Animals with incorrect injection sites or dialysis probe placements were removed from the study. 


\section{Intra-mPFC APDC and Acute Behavioral Response to Cocaine}

Figure 2-2A illustrates the dose response effect of intracortical APDC injection on the acute motor-stimulant response to cocaine (15 mg/kg i.p.). Acute cocaine administration (saline/cocaine) significantly increased locomotor activity, an effect that was reduced by intra-mPFC pre-treatment with the GGluR $_{2 / 3}$ agonist APDC (APDC/cocaine). It is shown in Figure 2-2A that the lowest dose of intra-mPFC APDC that significantly reduced cocaine induced locomotor activity was 1.5 $\mathrm{nmol} /$ side. Intracortical injection of APDC did not alter the locomotor response to a peripheral saline ( $1 \mathrm{ml} / \mathrm{kg}$ i.p.) injection (APDC/saline), suggesting that the inhibitory effects of APDC were specific to cocaine-induced behavioral effects. Base on the results of these dose response studies, $1.5 \mathrm{nmol} /$ side was used in the following studies.

Figure 2-2B illustrates the effects of intracortical co-injection of APDC and LY341495, which is an antagonist of $\mathrm{mGluR}_{2 / 3}$ receptor on the acute motor-stimulant response to cocaine $(15 \mathrm{mg} / \mathrm{kg}$ i.p.). Acute cocaine administration (saline/cocaine) significantly increased locomotor activity, an effect that was significantly reduced by intra-mPFC pre-treatment with the mGluR $_{2 / 3}$ agonist APDC (APDC/cocaine 1.5 $\mathrm{nmol} / \mathrm{side}$ ), but not altered by LY341495 alone (LY341495/cocaine $1.5 \mathrm{nmol} / \mathrm{side}$ ). However, when co-injected into the mPFC, LY341495 prevented the inhibitory effects of APDC on the acute motor-stimulant response to cocaine (APDC+LY341495/cocaine). Like APDC, intracortical injection of LY341495 did not alter the locomotor response to a peripheral saline $(1 \mathrm{ml} / \mathrm{kg}$ i.p. $)$ injection (LY341495/saline).

\section{Intra-mPFC APDC Injections and Initiation of Cocaine Sensitization}

Following the acute cocaine study, we examined the role of $\mathrm{mGluR}_{2 / 3}$ receptor in the development of cocaine sensitization. First, the initiation of cocaine-induced behavioral sensitization was determined. (Figure 2-3A) On day 1, animals received 'sham dialysis' in the experimental chambers. Following day 1, animals received the same treatment for three consecutive days in their home cages (see Table 2-1). Seven days later (day 11) in vivo microdialysis studies were conducted, during which all animals received cocaine challenge injection. The results showed that cocaine challenge injection induced a sensitized locomotor response during the first $60 \mathrm{~min}$ after intracortical saline injection in animals previously exposed to cocaine (saline/cocaine) as compared to animals receiving cocaine for the first time (saline/saline). However, the animals that received repeated intracortical APDC injections before each of their daily cocaine injections (APDC/cocaine) did not show an enhanced behavioral response to a challenge injection of cocaine. Furthermore, the behavioral responses of these animals were not significantly different from the animals receiving cocaine for the first time [(saline/saline) or (APDC/saline)]. 
(A) Dose Response Study

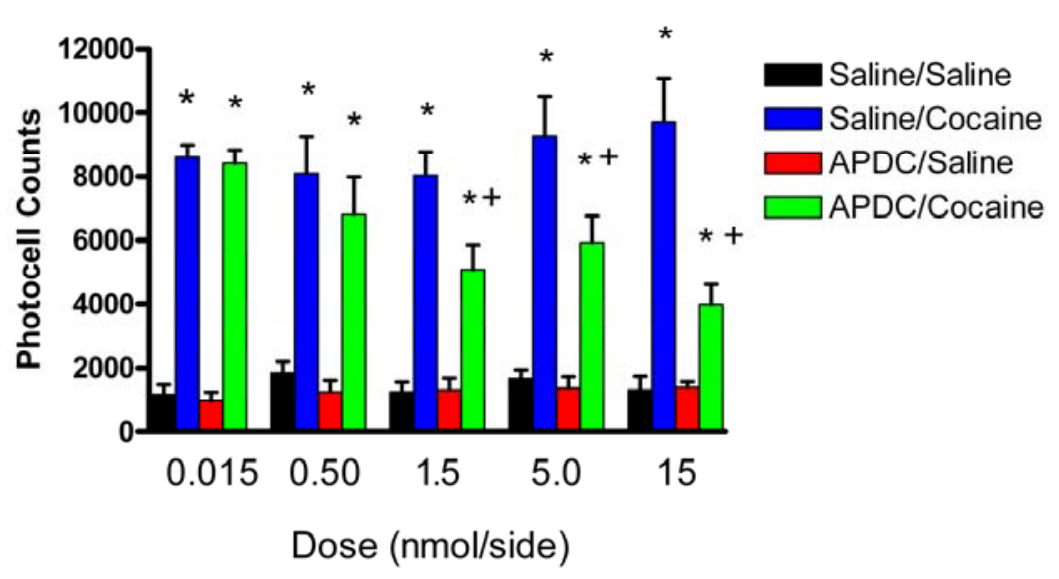

(B)

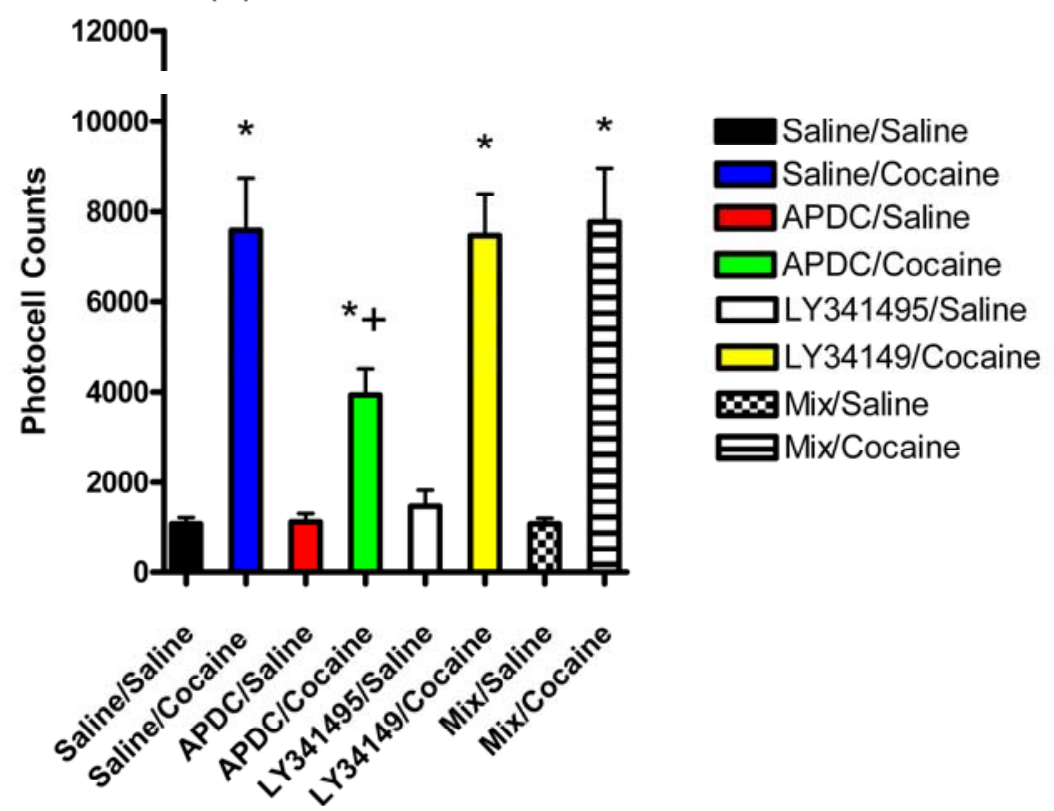

Figure 2-2. The effects of intra-mPFC injection of APDC on the acute motor response to cocaine $(15 \mathrm{mg} / \mathrm{kg}$ i.p.). The data are expressed as mean photocell counts $\pm \mathrm{SEM}$ for (A) dose response study and (B) APDC and LY341495 co-injection.

$\mathrm{Mix}=\mathrm{APDC}+\mathrm{LY} 341495$. The significance of the differences between treatment groups was determined by one-way ANOVA with repeated-measures followed by a student Newman-Keuls test. $(A){ }^{*} P<0.05$ compared to saline/saline and ${ }^{+} P<0.05$ compared to saline/cocaine. $(B) * P<0.05$ compared to saline/saline and ${ }^{+} P<0.05$ compared to cocaine saline/cocaine. The $\mathrm{F}$ scores from one way ANOVA were as followed: (A) $0.015 \mathrm{nmol} /$ side $\mathrm{F}(3,23)=117.5 \mathrm{P}<0.0001 ; 0.5 \mathrm{nmol} /$ side $\mathrm{F}(3,31)=12.87 \mathrm{P}<0.0001 ; 1.5$ $\mathrm{nmol} /$ side $\mathrm{F}(3,30)=33.93 \mathrm{P}<0.0001 ; 5 \mathrm{nmol} / \mathrm{side} \mathrm{F}(3,31)=23.88 \mathrm{P}<0.0001 ; 15$ $\mathrm{nmol} /$ side $\mathrm{F}(3,30)=27.25 \mathrm{P}<0.0001$. (B) $\mathrm{F}(7,92)=20.09 \mathrm{P}<0.0001$. 


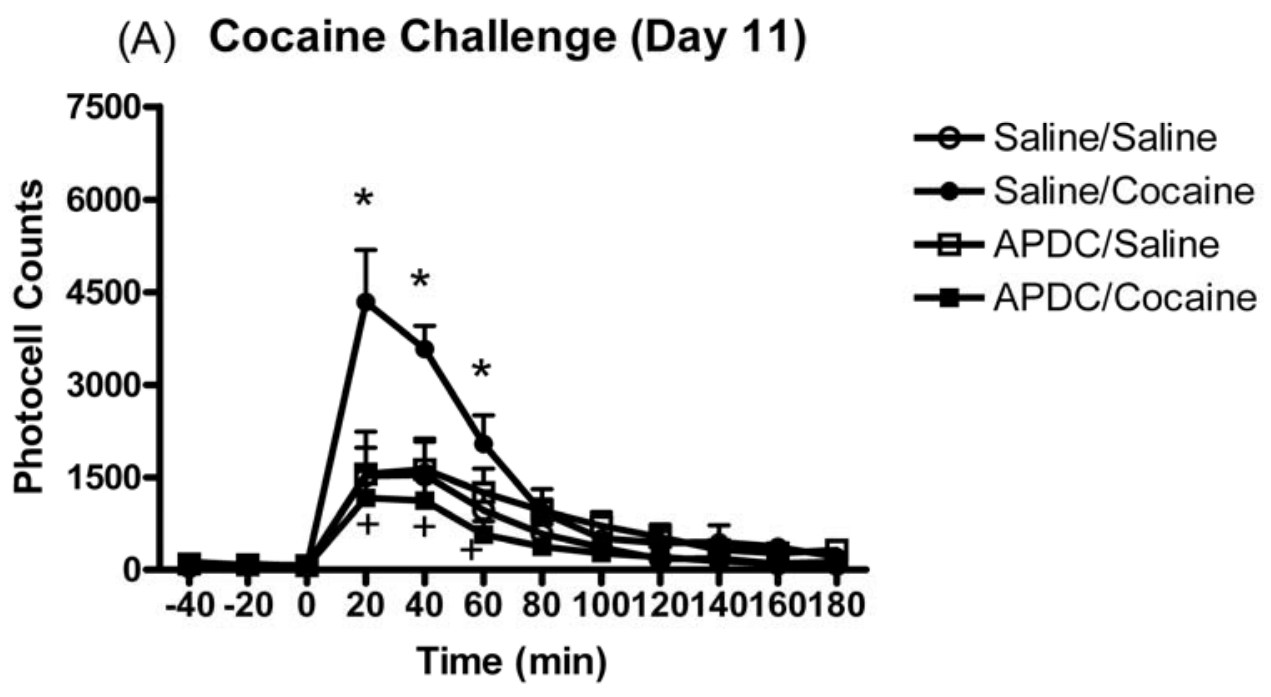

(B) Cocaine Challenge (Day 11)

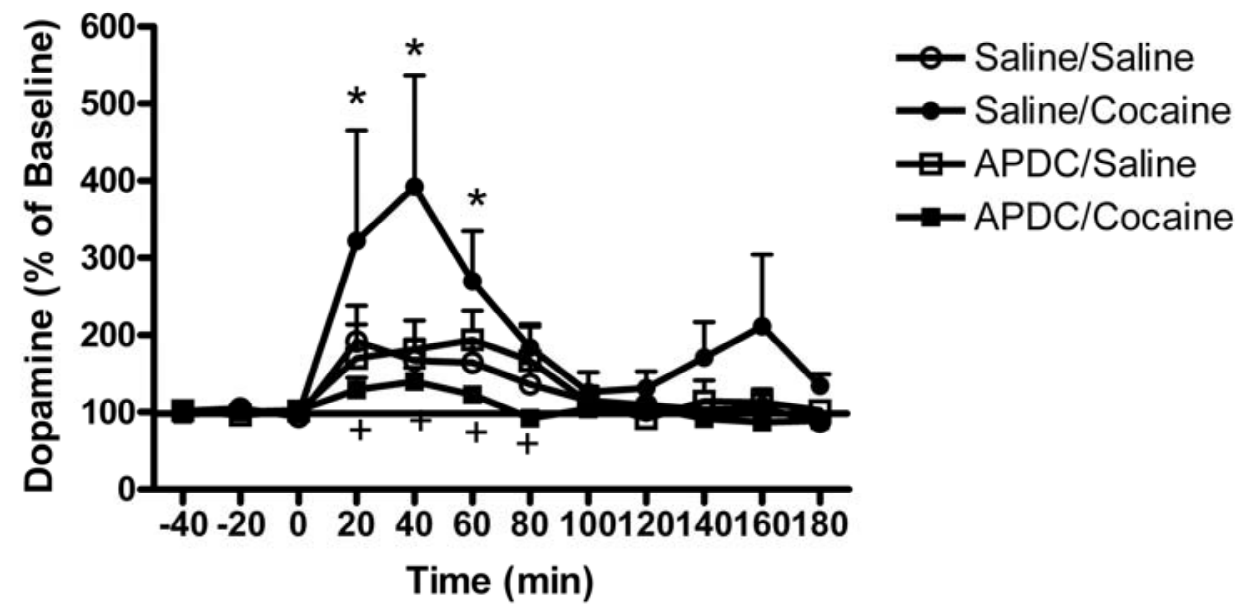

Figure 2-3. The effects of intra-mPFC APDC ( $1.5 \mathrm{nmol} / \mathrm{side})$ injection on the initiation of behavioral and neurochemical sensitization to cocaine $(15 \mathrm{mg} / \mathrm{kg}$ i.p. $)$. The data are expressed as mean photocell counts \pm SEM for $(A)$ last day of exposure to cocaine. The measurements of DA were made following the cocaine challenge and are expressed as mean percent of baseline \pm SEM. (B). The significance of the differences between treatment groups was determined by two-way ANOVA with repeated-measures followed by post hoc analysis using a modified least significant differences test (Milliken and Johnson, 1984). F scores were as follows: $(A)$ Treatment effect $\mathrm{F}(3,25)=4.498, \mathrm{p}=0.0122$; Time effect $\mathrm{F}(11,25)=33.993, \mathrm{p}<0.0001$; and Interaction effect $F(33,275)=3.944, p<0.0001$. (B) Treatment effect $F(3,25)=4.635$, $\mathrm{p}=0.0104$; Time effect $\mathrm{F}(11,25)=8.21, \mathrm{p}<0.0001$; and Interaction effect $\mathrm{F}(33,275)=1.688, \mathrm{p}=0.0135$. (A) $* P<0.05$ compared to saline/saline and ${ }^{+} P<0.05$ compared to saline/cocaine. $(B) * P<0.05$ compared to saline/saline and ${ }^{+} P<0.05$ compared to cocaine saline/cocaine. 
The effects of intracortical APDC injection on the initiation of neurochemical sensitization are illustrated in Figure 2-3B. Baseline dopamine concentrations were not significantly different between any of the groups. $[\mathrm{F}(3,25)=0.557, \mathrm{p}=0.6482]$ The baseline for the four groups are as followed: (saline/saline) $=40 \pm 5 \mathrm{fmol} / 20 \mathrm{~min}$; (saline $/$ cocaine) $=34 \pm 6 \mathrm{fmol} / 20 \mathrm{~min} ;($ APDC/saline $)=34 \pm 5 \mathrm{fmol} / 20 \mathrm{~min}$; (APDC/cocaine) $=43 \pm 5 \mathrm{fmol} / 20 \mathrm{~min}$. A peripheral challenge injection of cocaine significantly increased DA concentrations in the NAc of animals previously exposed to cocaine (saline/cocaine) as compared to animals receiving cocaine for the first time (saline/saline). However, the animals that received repeated intra-mPFC APDC injections before each of their daily injections of cocaine (APDC/cocaine) did not show a significantly augmented dopaminergic response to a cocaine challenge. Furthermore, this response was not significantly different from animals receiving cocaine for the first time [(saline/saline) and (APDC/saline)].

\section{Intra-mPFC APDC Injections and Expression of Cocaine Sensitization}

These studies were designed to demonstrate the ability of intra-mPFC APDC to modulate the expression of cocaine sensitization after 1 day, 7 day and 30 day withdrawal. On day 1, animals received 'sham dialysis' in the experimental chambers. Over the next three consecutive days, animals received same treatment in their home cages. The treatment regimen is described in Table 2-1.

Figures 2-4, 2-5 and 2-6 illustrate the effects of APDC on the expression of cocaine-induced behavioral and neurochemical sensitization following repeated cocaine pretreatment after 1 day, 7 days and 30 days respectively. In all the withdrawal time points, a cocaine challenge injection evoked a sensitized locomotor response in animals (saline/cocaine) previously exposed to cocaine after saline intracortical injection when compared to control animals (saline/saline) receiving their first cocaine injection. Intra-mPFC APDC injection before a cocaine challenge injection (APDC/cocaine) blocked the expression of the sensitized response in animals previously exposed to cocaine after 1 day withdrawal (Figure 2-4A). However, this effect was no longer apparent following 7 days (Figure 2-5A) and 30 days (Figure 2-6A) withdrawal. Interestingly, it was not consistently observed that intra-mPFC APDC injection significantly reduced the locomotor response in animals receiving cocaine for the first time (APDC/saline) as compared to control animals (saline/saline), an effect that we observed in the previous acute cocaine study. Intra-mPFC APDC injection attenuated the acute cocaine response in animals of 7 days and 30 days withdrawal groups but not 1 day withdrawal group.

The expression of neurochemical sensitization after 1 day, 7 days and 30 days withdrawal are shown in Figure 2-4B, Figure 2-5B and Figure 2-6B respectively. Baseline dopamine concentrations were not significantly different between any of the groups. 


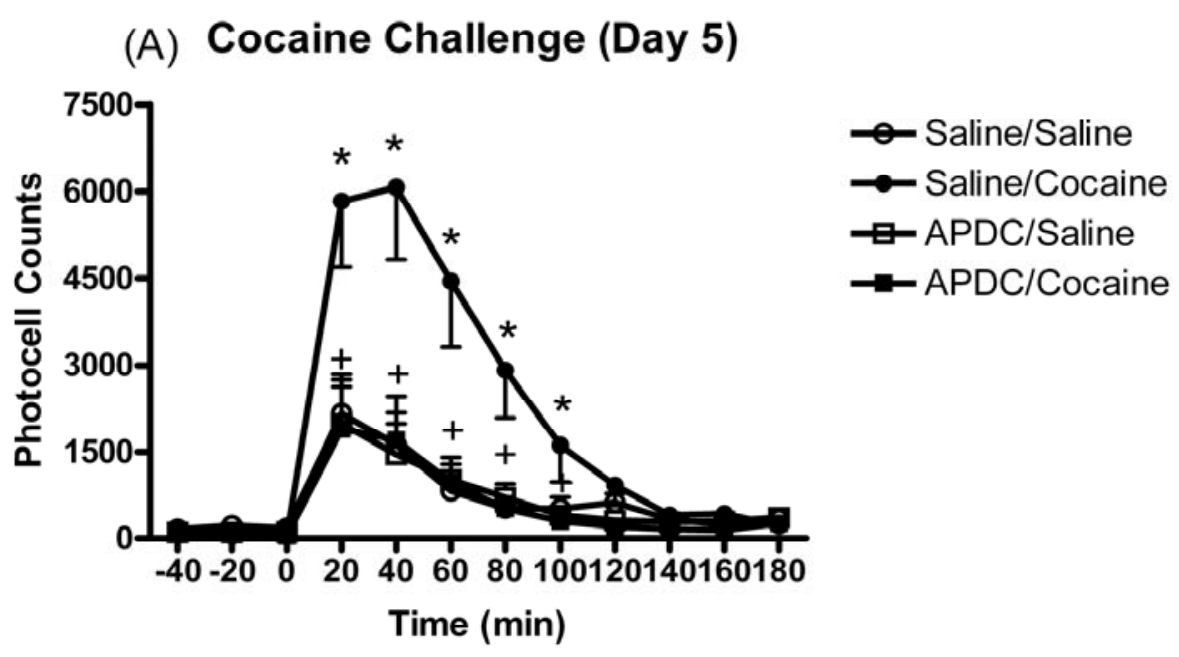

(B) Cocaine Challenge (day 5)

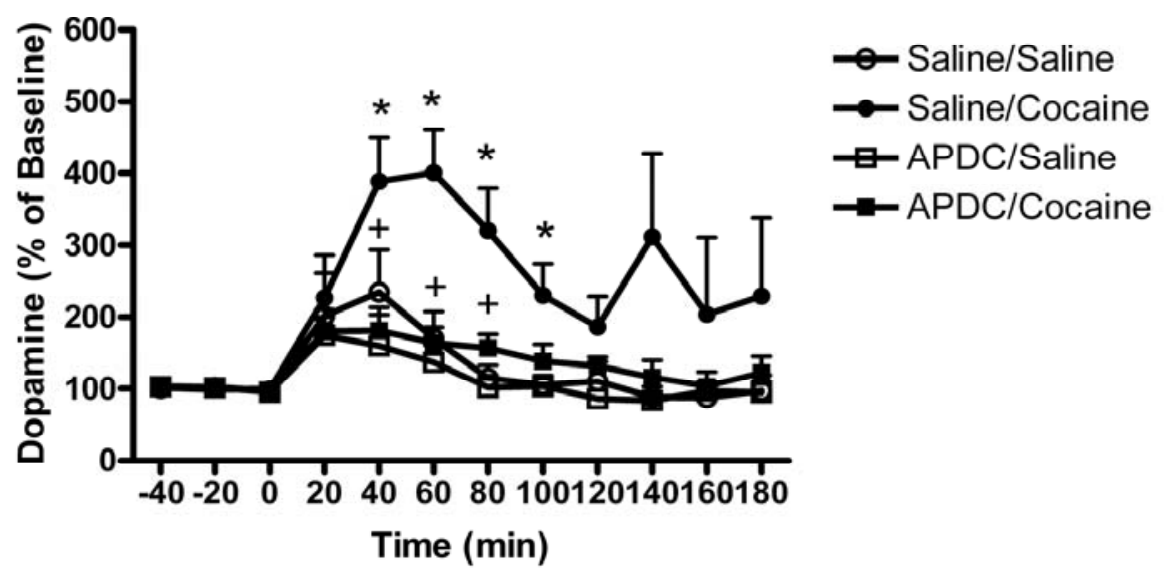

Figure 2-4. The effects of intra-mPFC APDC (1.5 nmol/side) injection on the expression of behavioral and neurochemical sensitization to cocaine (15 mg/kg i.p.) after 1 day withdrawal. The data are expressed as mean photocell counts \pm SEM for (A) last day of exposure to cocaine. The measurements of DA were made following the cocaine challenge and are expressed as mean percent of baseline \pm SEM. (B). The significance of the differences between treatment groups was determined by two-way ANOVA with repeated-measures followed by post hoc analysis using a modified least significant differences test (Milliken and Johnson, 1984). F scores were as follows: (A) Treatment effect $F(3,21)=7.012, p=0.0019$; Time effect $F(11,21)=31.332, p<0.0001$; and Interaction effect $F(33,231)=5.632, p<0.0001$. (B) Treatment effect $F(3,21)=6.451$, $\mathrm{p}=0.0029$; Time effect $\mathrm{F}(11,21)=7.879, \mathrm{p}<0.0001$; and Interaction effect $\mathrm{F}(33,231)=1.887, \mathrm{p}=0.0038$. (A) ${ }^{*} P<0.05$ compared to saline/saline and ${ }^{+} P<0.05$ compared to saline/cocaine. $(B) * P<0.05$ compared to saline/saline and ${ }^{+} P<0.05$ compared to cocaine saline/cocaine. 

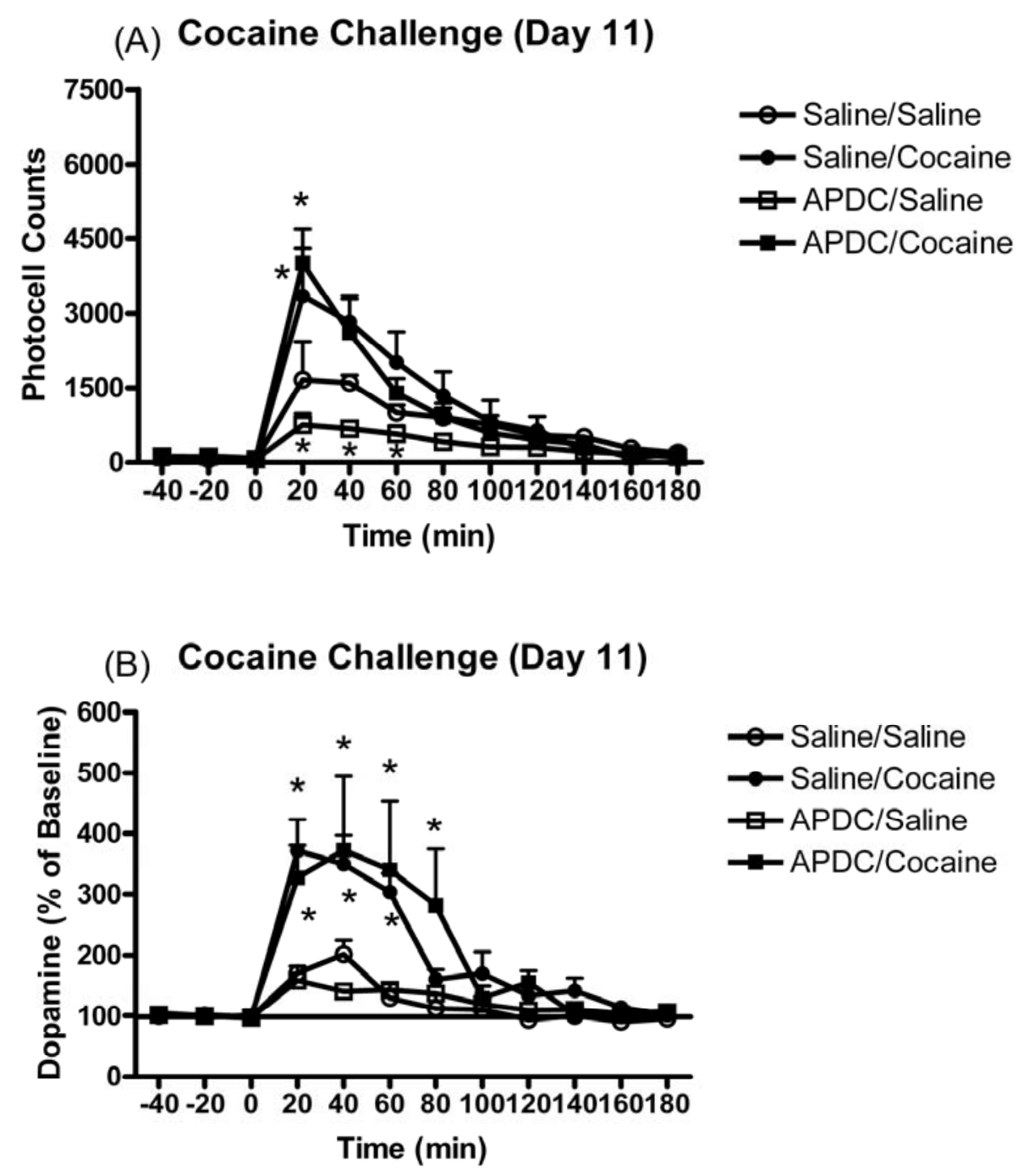

Figure 2-5. The effects of intra-mPFC APDC (1.5 nmol/side) injection on the expression of behavioral and neurochemical sensitization to cocaine (15 mg/kg i.p.) after 7 day withdrawal. The data are expressed as mean photocell counts $\pm S E M$ for (A) last day of exposure to cocaine. The measurements of DA were made following the cocaine challenge and are expressed as mean percent of baseline \pm SEM. (B). The significance of the differences between treatment groups was determined by two-way ANOVA with repeated-measures followed by post hoc analysis using a modified least significant differences test (Milliken and Johnson, 1984). F scores were as follows: (A) Treatment effect $\mathrm{F}(3,20)=5.161, \mathrm{p}=0.0084$; Time effect $\mathrm{F}(11,20)=45.261, \mathrm{p}<0.0001$; and Interaction effect $F(33,220)=4.964, p<0.0001$. (B) Treatment effect $F(3,20)=4.914$, $\mathrm{p}=0.0102$; Time effect $\mathrm{F}(11,20)=18.099, \mathrm{p}<0.0001$; and Interaction effect $\mathrm{F}(33,220)=3.096, \mathrm{p}<0.0001$. (A) ${ }^{*} P<0.05$ compared to saline/saline and ${ }^{+} P<0.05$ compared to saline/cocaine. $(B) * P<0.05$ compared to saline/saline and ${ }^{+} P<0.05$ compared to cocaine saline/cocaine. 


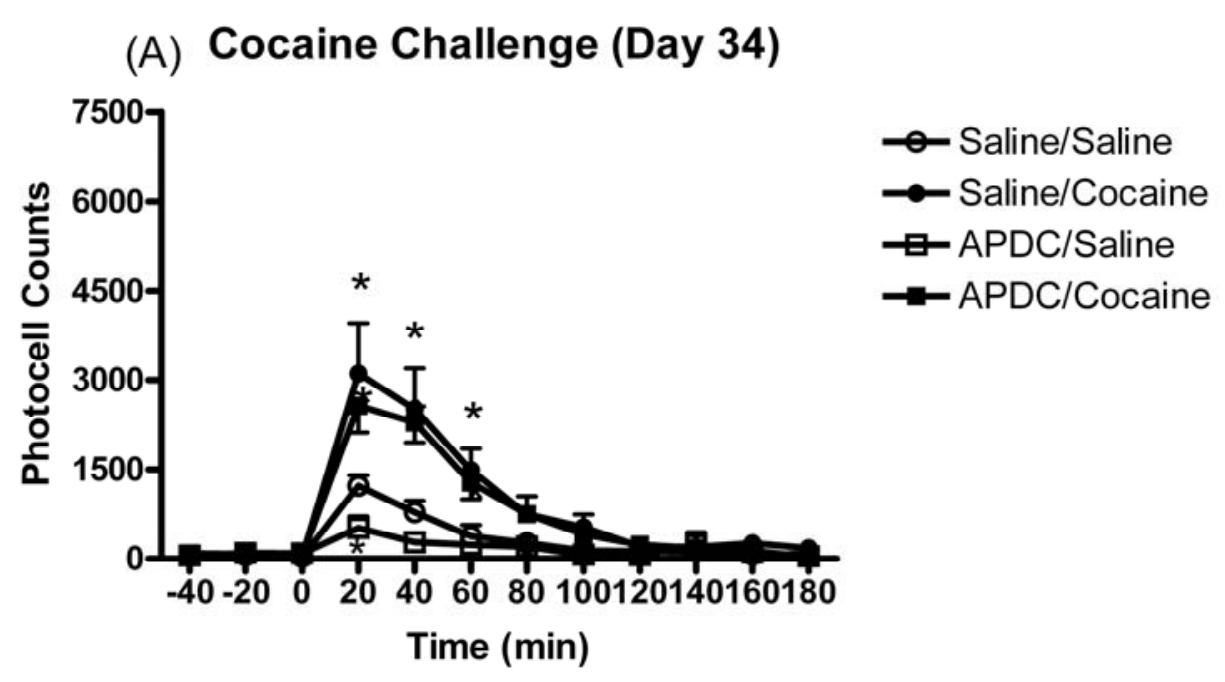

(B) Cocaine Challenge (Day 34)

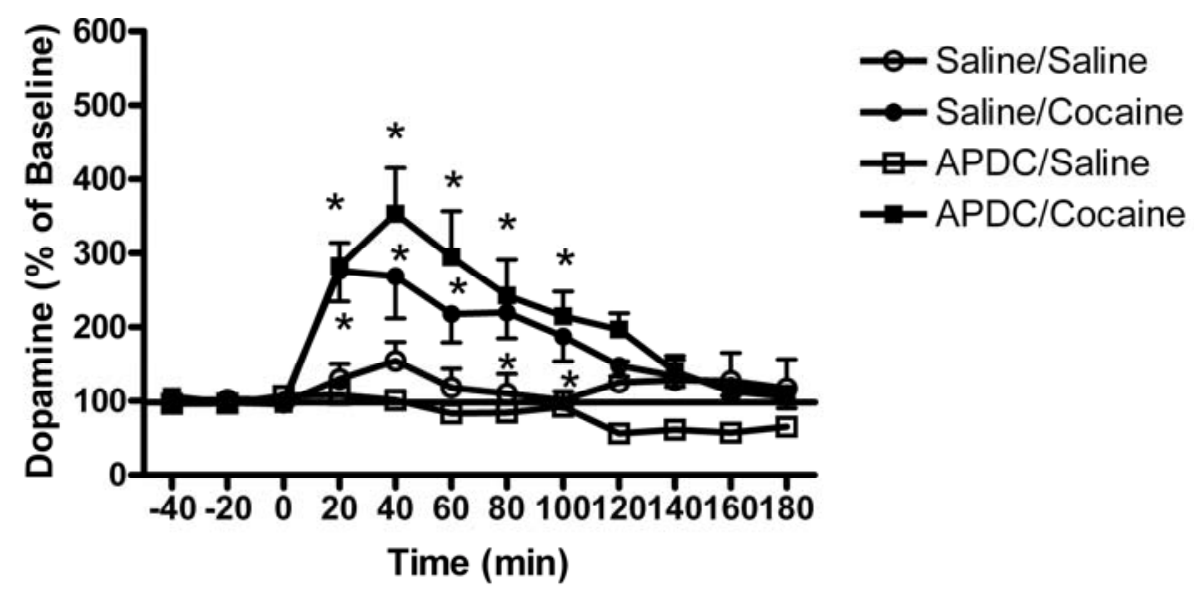

Figure 2-6. The effects of intra-mPFC APDC (1.5 nmol/side) injection on the expression of behavioral and neurochemical sensitization to cocaine (15 mg/kg i.p.) after 30 day withdrawal. The data are expressed as mean photocell counts \pm SEM for (A) last day of exposure to cocaine. The measurements of DA were made following the cocaine challenge and are expressed as mean percent of baseline \pm SEM. (B). The significance of the differences between treatment groups was determined by two-way ANOVA with repeated-measures followed by post hoc analysis using a modified least significant differences test (Milliken and Johnson, 1984). F scores were as follows: $(A)$ Treatment effect $\mathrm{F}(3,18)=8.062, \mathrm{p}=0.0013$; Time effect $\mathrm{F}(11,18)=34.892, \mathrm{p}<0.0001$; and Interaction effect $\mathrm{F}(33,198)=4.088, \mathrm{p}<0.0001$. (B) Treatment effect $\mathrm{F}(3,18)=6.512$, $\mathrm{p}=0.0036$; Time effect $\mathrm{F}(11,18)=15.143, \mathrm{p}<0.0001$; and Interaction effect $\mathrm{F}(33,198)=3.441, \mathrm{p}<0.0001$. (A) $* P<0.05$ compared to saline/saline and ${ }^{+} P<0.05$ compared to saline/cocaine. $(B) * P<0.05$ compared to saline/saline and ${ }^{+} P<0.05$ compared to cocaine saline/cocaine. 
The baseline for the four groups in three experimental groups are as follows: 1 day withdrawal $[\mathrm{F}(3,21)=0.657, \mathrm{p}=0.5876]$ (saline $/$ saline $)=36 \pm 5 \mathrm{fmol} / 20 \mathrm{~min}$; (saline $/$ cocaine $)=30 \pm 5 \mathrm{fmol} / 20 \mathrm{~min} ;($ APDC/saline $)=29 \pm 6 \mathrm{fmol} / 20 \mathrm{~min}$; $(\mathrm{APDC} /$ cocaine $)=39 \pm 7 \mathrm{fmol} / 20 \mathrm{~min} .7$ days withdrawal $[\mathrm{F}(3,20)=0.132, \mathrm{p}=0.94]$ (saline $/$ saline $)=46 \pm 5 \mathrm{fmol} / 20 \mathrm{~min}$; (saline $/$ cocaine $)=47 \pm 5 \mathrm{fmol} / 20 \mathrm{~min}$; $($ APDC/saline $)=49 \pm 8 \mathrm{fmol} / 20 \mathrm{~min} ;($ APDC/cocaine $)=44 \pm 8 \mathrm{fmol} / 20 \mathrm{~min} .30$ days withdrawal $[\mathrm{F}(3,21)=2.141, \mathrm{p}=0.1254]$ (saline $/$ saline $)=32 \pm 7 \mathrm{fmol} / 2 \mathrm{0min}$; (saline $/$ cocaine $)=22 \pm 5 \mathrm{fmol} / 20 \mathrm{~min} ;($ APDC/saline $)=48 \pm 12 \mathrm{fmol} / 20 \mathrm{~min}$; $($ APDC/cocaine $)=28 \pm 5 \mathrm{fmol} / 20 \mathrm{~min}$. Consistent with the behavioral study, a cocaine challenge produced a sensitized DA response in the NAc (saline/cocaine) in animals previously exposed to cocaine. This effect was attenuated in rats that received an intra-mPFC APDC injection before their peripheral challenge injection of cocaine after 1 day withdrawal (APDC/cocaine) (Figure 2-4B).However, this effect was no longer apparent following 7 days and 30 days withdrawal (Figure 2-5B and Figure2-6B respectively). It should be noted that intra-mPFC APDC injection produced a non-significant trend towards reducing acute cocaine-induced DA concentrations in the NAc (APDC/saline) of all three experimental groups.

\section{Discussion}

The present study demonstrated that intra-mPFC microinjection of APDC reduced the acute motor-stimulant response as well as the initiation of behavioral and neurochemical sensitization to cocaine. Once sensitization was induced, intracortical administration of APDC blocked cocaine-induced behavioral and neurochemical responses in sensitized animals after 1 day of withdrawal, but not after 7 or 30 days of withdrawal. Therefore, the main conclusion from the present study is that the ability of $\mathrm{mGluR}_{2 / 3}$ receptors in the $\mathrm{mPFC}$ to modulate behavioral and neurochemical responses to cocaine is reduced in cocaine-sensitized animals.

\section{Medial Prefrontal Cortex and Acute Motor Activity}

The glutamatergic pyramidal neurons are the major output neurons in the mPFC. It has been demonstrated that these pyramidal neurons send significant afferents to many subcortical brain regions including the NAc and VTA. (Sesack et al. 1989; Takagishi and Chiba 1991; Pierce and Kalivas 1997) Electrical stimulation of the medial prefrontal cortex induces glutamate release in the ventral tegmental area (VTA) of freely moving animals (Rossetti et al. 1998). In addition, electrical stimulation of the medial prefrontal cortex increases dopamine release in the striatum (Taber and Fibiger 1993). Previous studies have shown a significant positive correlation between DA in the NAc and locomotor activity after systemic administration of cocaine (Hedou et al. 1999). Therefore, by providing drive to subcortical brain regions in the mesolimbic DA system, which plays an important role in governing locomotor 
activity, (Steketee et al. 1992; Tzschentke 2001) the mPFC is obviously in a position to modulate motor behavior.

Studies have reported the neurotransmission in the mPFC can affect acute motor activity induced by psychostimulants. Intra-mPFC microinjections of quinpirole, a $\mathrm{DA} \mathrm{D}_{2 \text {-like }}$ agonist and baclofen, which is a $\mathrm{GABA}_{\mathrm{B}}$ agonist can dose-dependently block acute cocaine induced motor activity (Beyer and Steketee 2000; Steketee and Beyer 2005). Besides DA and GABA, glutamate modulation of pyramidal neurons is also well studied in mPFC. Glutamate terminals mainly come from mediodorsal nucleus of the thalamus, the hippocampus and the amygdala. In addition, mPFC pyramidal neurons have recurrent collaterals that synapse on other pyramidal neurons located within this region (Sesack et al. 1989; Pirot et al. 1994; Bacon et al. 1996; Jay et al. 1996; Steketee 2005). Glutamate released from these terminals may act on glutamate receptors to influence neurotransmission in the mPFC. Thus, pharmacological agents may influence the acute motor response to psychostimulants by directly affecting these glutamate receptors. In the present study, it was found that intra-mPFC injection of $\mathrm{mGluR}_{2 / 3}$ agonist $\mathrm{APDC}$ reduced the acute motor response to cocaine. The selectivity of APDC was verified by co-injection of APDC plus LY341495, which is an antagonist of $\mathrm{mGluR}_{2 / 3}$ receptor. Therefore, activation of $\mathrm{mGluR}_{2 / 3}$ receptors in the $\mathrm{mPFC}$ reduces the acute motor response to cocaine. It was also found that intra-mPFC injection of APDC or LY341495 did not significantly alter the motor responses to saline, suggesting that the spontaneous locomotor activity differs markedly from that induced by psychostimulants, such as cocaine.

Notably, intra-mPFC APDC ( $1.5 \mathrm{nmol} / \mathrm{side})$ was able to significantly reduce the acute motor-stimulant response to cocaine in the initial dose-response studies, however this result was not consistently replicated in the studies of the effects of intra-mPFC APDC on the expression of sensitization. Desensitization of $\mathrm{mPFC}$ $\mathrm{mGluR}_{2 / 3}$ receptors caused by stress associated with daily pretreatment of saline injections and restraints could be one possible explanation for this effect. Acute stress can induce glutamate release in mPFC. (Moghaddam 2002) Therefore, repeated increases in glutamate transmission induced by repeated stress exposures could induce $\mathrm{mGluR}_{2 / 3}$ receptor desensitization, which may explain the reduced effects of intra-mPFC APDC in sensitization studies.

Taken together, these studies, combined with other recent reports, suggest that excitatory transmission from the $\mathrm{mPFC}$ to subcortical dopaminergic brain regions play an important role in psychostimulant-mediated behaviors, and activation of $\mathrm{mGluR}_{2 / 3}$ can reduce the acute motor response to psychostimulant, such as cocaine. 


\section{Cortical Excitatory Transmission and Behavioral Sensitization}

The critical role of the medial prefrontal cortex (mPFC) in the development of cocaine-induced sensitization has been shown in many research reports. Moreover, accumulating evidence has shown that the alterations of various MPFC neurotransmitter systems, including dopamine, serotonin, glutamate, noradrenaline, acetylcholine, GABA, and neuropeptides, including their receptors may contribute to the development of cocaine sensitization and addiction. (Steketee 2003) More specifically, previous studies suggested that altered modulation of mPFC pyramidal neurons is involved in the mechanisms for sensitization. Repeated cocaine administration increased the excitability of $\mathrm{mPFC}$ neurons possibly via reducing the activity of the potassium inward rectifiers and voltage-gated $\mathrm{K}^{+}$currents as long as 3 weeks after withdrawal. (Nasif et al. 2005) In addition, chronic exposure to cocaine facilitates the responsiveness of $\mathrm{Ca}^{2+}$ currents, particularly via the activated L-type

$\mathrm{Ca}^{2+}$ channels, to excitatory stimuli in rat mPFC pyramidal neurons (Nasif et al. 2005). Consistent with these findings, it has been shown that repeated cocaine exposure produced time-dependent increases in cocaine-mediated $\mathrm{mPFC}$ glutamate overflow, suggesting that transient increases in cocaine-induced glutamate levels in the mPFC during the first week of withdrawal may play an important role in the development of behavioral sensitization to cocaine. (Williams and Steketee 2004) Excitatory output from the $\mathrm{mPFC}$ could result from an increase in excitatory transmission via ionotropic receptors, such as increased Ser845 phosphorylation of the GluR1 subunit in prefrontal cortex. (Zhang et al. 2007) Also a decrease in inhibitory glutamate transmission via group II metabotropic glutamate receptors $\left(\mathrm{mGluR}_{2 / 3}\right)$ may result in enhanced excitatory output from the mPFC. Therefore, our study was designed to demonstrate the potential role of $\mathrm{mPFC} \mathrm{mGluR}_{2 / 3}$ receptor in sensitization.

\section{Cortical mGluR $R_{2 / 3}$ Receptors and Behavioral Sensitization}

Specific locations for $\mathrm{mGluR}_{2 / 3}$ receptors remain to be identified in the $\mathrm{mPFC}$, however, it is reasonable to assume that $\mathrm{mGluR}_{2 / 3}$ receptors are localized on the glutamatergic terminals and on pyramidal neurons, which is supported by an electrophysiological study that has shown postsynaptic prefrontal cortical $\mathrm{mGluR}_{2 / 3}$ receptor activation can induce LTD of pyramidal neurons. (Otani et al. 2002) Therefore, it is possible that intra-mPFC injection of $\mathrm{mGluR}_{2 / 3}$ receptor agonist APDC, by inhibiting excitatory output from this region, could prevent cocaine-induced motor activity as well as the initiation of sensitization as was seen in the present study. However, once sensitization was induced, APDC was no longer capable of modulating the behavioral response to cocaine following prolonged withdrawal from cocaine. These data suggest that repeated exposure to cocaine decreases $\mathrm{mGluR}_{2 / 3}$ receptor function, consistent with previous studies suggesting the functional loss of $\mathrm{mGluR}_{2 / 3}$ receptors in NAc and $\mathrm{mPFC}$ after repeated cocaine exposure (Xi et al. 2002; Bowers et al. 2004). Taken together, these results suggest that the loss of function of $\mathrm{mGluR}_{2 / 3}$ receptor is not restricted to one brain region, but most possibly distributed 
within the mesocorticolimbic area.

The loss of function of $\mathrm{mGluR}_{2 / 3}$ receptor is due to the reduction of receptor coupling rather than receptor numbers. (Xi et al. 2002) Moreover, chronic cocaine administration reduces $\mathrm{G}$ protein signaling efficacy in $\mathrm{PFC}$ at least in part by increasing the expression of AGS3, which binds to GiaGDP and inhibits GDP dissociation. The cocaine-induced reduction in Gi $\alpha$ coupling to $\mathrm{mGluR}_{2 / 3}$ could be reversed by infusing AGS3 antisense into PFC. (Bowers et al. 2004) In addition, AGS3 expression was not elevated during early withdrawal, (Bowers et al. 2004) which might in part explain why APDC blocked the expression of sensitization after 1 day withdrawal in the present study.

\section{Summary}

In conclusion, the present study demonstrated that intra-mPFC APDC prevented the acute cocaine induced motor response and initiation of the sensitized motor stimulant responses to cocaine. However the ability of intra-mPFC APDC to block cocaine induced behavior was lost in sensitized animals after prolonged, but not short term withdrawal. These data provide additional support for the idea that sensitization to cocaine results from an increase in excitatory output from the mPFC to subcortical regions, which in part results from the disinhibition of $\mathrm{mPFC}$ pyramidal neurons. (Steketee 2005) The results from the present study are similar to previous studies examining the role of $\mathrm{mPFC}$ dopamine $\mathrm{D}_{2}$ and $\mathrm{GABA}_{\mathrm{B}}$ receptors in the development of sensitization. Infusions of the dopamine $\mathrm{D}_{2}$ agonist quinpirole or the $\mathrm{GABA}_{B}$ agonist baclofen into the $\mathrm{mPFC}$ blocked the initiation of sensitization to cocaine. However, once sensitization was induced, intra-mPFC quinpirole was only able to attenuate, while baclofen failed to alter, the expression of sensitization. (Beyer and Steketee 2002; Steketee and Beyer 2005) This finding is supported by recent findings which demonstrated that repeated cocaine exposure reduces $\mathrm{mGluR}_{2 / 3}$ responsiveness at $\mathrm{mPFC}$ excitatory synapses after 5 day withdrawal (Huang et al. 2007). 


\section{Chapter 3. Repeated Exposure to Cocaine Alters the Modulation of Mesocorticolimbic Glutamate Transmission by Medial Prefrontal Cortex Group II Metabotropic Glutamate Receptors}

\section{Introduction}

Repeated cocaine exposure enhances glutamatergic output from medial prefrontal cortex (mPFC) to subcortical brain regions, including the nucleus accumbens (NAc) and ventral tegmental area (VTA). The enhanced glutamatergic output is associated with augmented behavioral responses to cocaine challenge, which is referred as behavioral sensitization (Pierce et al. 1996; Wolf 1998; Tzschentke 2000; Steketee 2003; Kalivas 2004). It has been suggested that the enhanced glutamatergic output may result from increased excitatory drive in the mPFC. For example, a transient increase of glutamate overflow was observed in cocaine sensitized animals after 1 day and 7 days of withdrawal but not 30 days of withdrawal (Williams and Steketee 2004). Repeated cocaine administration increased membrane excitability of pyramidal neurons in the rat medial prefrontal cortex via an increase in voltage-sensitive calcium currents (Nasif et al. 2005). Besides the enhancement of excitatory drive, it has been hypothesized that loss of inhibitory control may also be responsible for the enhancement of glutamatergic output from mPFC (Steketee 2003). Previous studies have indicated the reduction of receptor function including dopamine $\mathrm{D}_{2}$ and $G A B A_{B}$, in the mPFC of cocaine sensitized animals (Beyer and Steketee 2002; Steketee and Beyer 2005). In addition, repeated cocaine administration has been shown to promote long-term potentiation induction in rat mPFC (Huang et al. 2007). Both $\mathrm{GABA}_{\mathrm{A}}$ receptor-mediated synaptic currents and $\mathrm{GABA}_{\mathrm{A}}$ alphal subunit expression in $\mathrm{mPFC}$ pyramidal neurons were reduced following repeated cocaine exposure (Huang et al. 2007). Thus, it was suggested that this promotion was caused by cocaine-induced reduction of $\mathrm{GABA}_{\mathrm{A}}$ receptor-mediated inhibition of $\mathrm{mPFC}$ pyramidal neurons (Huang et al. 2007). Consistent with previous studies, study in Chapter 2 indicated that repeated cocaine exposure reduced the ability of Group II metabotropic glutamate receptors $\left(\mathrm{mGluR}_{2 / 3}\right)$ to modulate the behavioral and neurochemical responses to cocaine after a long period of withdrawal, suggesting that the functional reduction of this inhibitory receptor is correlated with the long term expression of cocaine induced behavioral sensitization. Therefore, the functional reduction of these inhibitory receptors may cause the enhancement of mesocorticolimbic glutamate transmission in sensitized animals upon cocaine challenge.

$\mathrm{mGluR}_{2 / 3}$ receptors were indicated to play an inhibitory role in regulating glutamate release. $\mathrm{mGluR}_{2}$ and $\mathrm{mGluR}_{3}$ are found in various combinations of presynaptic, postsynaptic and glial localizations that may reflect differential modulation of excitatory amino acid transmission (Petralia et al. 1996; Conn and Pin 1997). Inhibition of presynaptic mGluR $_{2 / 3}$ receptors elevates extracellular glutamate 
level in the NAc (Xi et al. 2002). In addition to acting as an autoreceptor and/or postsynaptic inhibitory receptor, $\mathrm{mGluR}_{2 / 3}$ receptors can decrease extracellular glutamate levels in the NAc by inhibiting the $\mathrm{Na}^{+}$-independent cystine/glutamate antiporter (Baker et al. 2002; Baker et al. 2002; Baker et al. 2003). It has been shown that repeated cocaine administration reduced the function of $\mathrm{mGluR}_{2 / 3}$ receptors in the NAc (Xi et al. 2002). The functional reduction of $\mathrm{mGluR}_{2 / 3}$ receptors in the NAc may partly result in the enahanced glutamate release within this region associated with cocaine sensitization. However, few studies have been conducted to reveal the modulation of mesocorticolimbic glutamate transmission by $\mathrm{mPFC} \mathrm{mGluR}_{2 / 3}$ receptors. Thus, we designed experiments to examine the role of $\mathrm{mGluR}_{2 / 3}$ receptors in modulation of mesocorticolimbic glutamate transmission in both repeated saline and cocaine pretreated animals.

Studies have demonstrated a transient cocaine induced increase in mPFC glutamate overflow in sensitized animals (Williams and Steketee 2004) However, the source of the glutamate was not determined. Cystine/glutamate antiporters are also located on the cells in the mPFC (Melendez et al. 2005). Thus, glutamate released from both $\mathrm{mPFC}$ afferent terminals and axon collaterals of pyramidal neurons can include both vesicular and non-vesicular pools of glutamate. Intra-mPFC infusion of an $\mathrm{mGluR}_{2 / 3}$ receptor antagonist but not an agonist elevated the glutamate level in the mPFC (Melendez et al. 2005), suggesting that $\mathrm{mGluR}_{2 / 3}$ receptors may negatively regulate the glutamate release in the $\mathrm{mPFC}$. It has been shown that $\mathrm{K}^{+}$-stimulated glutamate efflux in the mPFC was enhanced in animals following repeated methamphetamine exposure (Stephans and Yamamoto 1995), suggesting that repeated cocaine exposure may influence the vesicular pool of glutamate. Recent evidence suggested that the non-vesicular releasable pool of glutamate regulated by the cystine/glutamate antiporter in the NAc was modulated by repeated cocaine administration (Baker et al. 2003). However, it has yet to be determined whether repeated cocaine exposure can alter the release of this pool of glutamate in the $\mathrm{mPFC}$ and whether the reduction of $\mathrm{mGluR}_{2 / 3}$ receptor may be one of the possible factors involved in alteration of this glutamate releasing pool in cocaine sensitized animals. Therefore, it is necessary to examine them.

Therefore, pharmacological agents of $\mathrm{mGluR}_{2 / 3}$ receptors including an agonist, LY379268 and/or an antagonist, LY341495 were chosen to functionally assess effects of repeated cocaine exposure on $\mathrm{mPFC} \mathrm{mGluR}_{2 / 3}$ receptors' modulation of mesocorticolimbic glutamate transmission during the development of cocaine sensitization. 


\section{Materials and Methods}

\section{Animals and Surgery}

Male Spraque-Dawley rats that weighed 275-300 g at the time of surgery, were housed under $12 \mathrm{hr}$ light/dark cycle and had free access to food and water. Rats were housed in groups of four before surgery and were individually housed after surgery. Prior to surgery, rats were anaesthetized with anesthetic (ketamine hydrochloride and xylazine: $80 / 12.5 \mathrm{mg} / \mathrm{kg}$ respectively; i.p.) and their heads were mounted in a stereotaxic frame (Kopf Instruments). The scalp was incised to expose the skull, and bregma and lambda were aligned in the same horizontal plane. All animals received microdialysis guide cannulae implanted $3.0 \mathrm{~mm}$ above the $\mathrm{mPFC}(\mathrm{A} / \mathrm{P},+3.2 \mathrm{~mm}$; $\mathrm{M} / \mathrm{L}, \pm 0.6 \mathrm{~mm}$ and $\mathrm{D} / \mathrm{V},-1.5 \mathrm{~mm}$ ) for dual probe-microdialysis experiments, in which animals also ipslaterally received implants of guide cannulae $3.0 \mathrm{~mm}$ above the NAc $(\mathrm{A} / \mathrm{P},+1.4 \mathrm{~mm} ; \mathrm{M} / \mathrm{L}, \pm 1.4 \mathrm{~mm}$ and $\mathrm{D} / \mathrm{V},-5.0 \mathrm{~mm})$ or VTA $(\mathrm{A} / \mathrm{P},-4.8 \mathrm{~mm} ; \mathrm{M} / \mathrm{L}$, $\pm 0.6 \mathrm{~mm}$ and $\mathrm{D} / \mathrm{V},-5.4 \mathrm{~mm})$. For single probe-microdialysis experiments of the present study, animals only received implant of 20 gauge $(14 \mathrm{~mm})$ microdialysis guide cannula $3.0 \mathrm{~mm}$ above the $\mathrm{mPFC}$. The $\mathrm{A} / \mathrm{P}, \mathrm{M} / \mathrm{L}$ and $\mathrm{D} / \mathrm{V}$ coordinates are based on bregma, midline and dura, respectively (Paxinos and Watson, 1997). Cannulae were anchored with 3 stainless steel screws and dental acrylic. Obturators (25 gauge, 14 $\mathrm{mm}$ ) were inserted into the cannulae in order to prevent their occlusion. Animals were allowed at least one week to recover from surgery. All procedures were conducted in accordance with the National Institutes of Health Guide for the Care and Use of Laboratory Animals. The University of Tennessee Health Science Center Animal Resources Advisory Committee approved all experimental procedures.

\section{In vivo Microdialysis}

Microdialysis experiments were conducted in conscious, freely moving rats. Animals received daily injections of saline $(1.0 \mathrm{ml} / \mathrm{kg})$ or cocaine $(15 \mathrm{mg} / \mathrm{kg})$ over 4 consecutive days in their home cages. In vivo microdialysis experiments were conducted 1, 7 or 30 days following the last of the daily injections of cocaine. Dialysis probes ( $2 \mathrm{~mm}$ active membrane) were introduced into the guide cannulae $16 \mathrm{hr}$ prior to the beginning of the dialysis experiments, in order to minimize damage-induced release of neurotransmitters and metabolites.

Experiment 1: $\mathrm{mGluR}_{2 / 3}$ receptor and mesocorticolimbic glutamate transmission The experiment was designed as follows: The probe was continuously perfused with dialysis buffer ( $\mathrm{KCl} 2.7 \mathrm{mM}, \mathrm{NaCl} 140 \mathrm{mM}, \mathrm{CaCl}_{2} 1.2 \mathrm{mM}, \mathrm{MgCl}_{2} 1.2 \mathrm{mM}$, plus 0.2 $\mathrm{mM}$ phosphate-buffered saline to achieve a $\mathrm{pH}=7.4$ ) at a flow rate of $2 \mu \mathrm{l} / \mathrm{min}$ during sample collection. The flow rate was reduced to $0.003 \mu \mathrm{l} / \mathrm{min}$ during the overnight period and was adjusted back to $2 \mu \mathrm{l} / \mathrm{min}$ at least $1 \mathrm{hr}$ before the beginning of sample collection. Following collection of four baseline samples, 
(2S)-2-Amino-2-[(1S,2S)-2-carboxycycloprop-1-yl]-3-(xan th-9-yl) propanoic acid (LY341495), a mGluR $2 / 3$ receptor antagonist, in escalating concentrations $(0.1,1$ and $10 \mu \mathrm{M}$ ), was infused into the mPFC via reverse dialysis Four samples were collected from the $\mathrm{mPFC}$, nucleus accumbens and/or VTA after each change in concentration. Samples were collected into $10 \mu \mathrm{l}$ of $0.05 \mathrm{mM} \mathrm{HCl}$ in $0.5 \mathrm{ml}$ microcentrifuge tubes. Samples either immediately underwent high performance liquid chromatography (HPLC) analysis of glutamate or were stored at $-80^{\circ} \mathrm{C}$. Samples were stored no more than 2 weeks.

Experiment 2: $\mathrm{mGluR}_{2 / 3}$ receptor and $\mathrm{mPFC}$ glutamate release The experiment was designed as follows: All the animals received single probe in the $\mathrm{mPFC}$. All the procedures were the same as the above except that animals received infusions of $\mathrm{KCl}(80 \mathrm{mM})$ or cystine $(50 \mu \mathrm{M})$ following collection of baseline samples. After collection of 4 additional samples, animals received intra-mPFC infusions of (1R,4R,5S,6R)-4-Amino-2-oxabicyclo[3.1.0]hexane-4,6-dic arboxylic acid (LY379268), an agonist of $\mathrm{mGluR}_{2 / 3}$ receptor with $\mathrm{KCl}(80 \mathrm{mM})$ or cystine (50 $\mu \mathrm{M})$ buffer via reverse dialysis for another 4 samples. Then, animals continued to receive infusions of dialysis buffer alone for the last 4 samples. In general, samples were collected every 20 minutes. Samples were collected into $10 \mu 1$ of $0.05 \mathrm{M} \mathrm{HCl}$ in $0.5 \mathrm{ml}$ microcentrifuge tubes. Samples either immediately underwent high performance liquid chromatography (HPLC) analysis of glutamate or were stored at $-80^{\circ} \mathrm{C}$. Samples were stored no more than 2 weeks.

\section{High Performance Liquid Chromatography}

The glutamate HPLC system consists of a $25 \mathrm{~cm}(5 \mu \mathrm{m})$ octadecasilane column, a Shimadzu LC-10AD solvent delivery system (Shimadzu corporation, Kyoto, Japan), a Shimadzu SIL-10AD autosampler and a fluorescence spectrophotometer (Shimadzu RF-10AXL). Samples underwent derivatization $(20 \mu 1$ sample $+20 \mu 1$ fluoraldehyde $)$ before being injected onto the column via the autosampler. The flow rate of the mobile phase $\left(62 \mathrm{mM} \mathrm{NaH}_{2} \mathrm{PO}_{4}, 0.5 \% \mathrm{v} / \mathrm{v}\right.$ tetrahydrofuran and $40 \% \mathrm{v} / \mathrm{v}$ methanol, $\mathrm{pH}$ $=6.3$ with $6 \mathrm{~N} \mathrm{NaOH}$ ) was $1.0 \mathrm{ml} / \mathrm{min}$. The fluorescence spectrophotometer detected glutamate with excitation wavelength of $260 \mathrm{~nm}$ and emission wavelength of $455 \mathrm{~nm}$. The external standard curve ranged from $0.5-25$ pmol for quantification.

\section{Behavior}

Animals' locomotor activity was measured in a micromax monitoring system (Accuscan Instruments, Columbus, OH, USA) as previously described (Beyer and Steketee 2002) to verify the treatment regimen produced sensitization. Within 3 days after the in vivo microdialysis experiments, animals were adapted to activity boxes $(45 \times 24 \times 19 \mathrm{~cm})$, which were set into individual sound attenuating chambers, for $60 \mathrm{~min}$. Then, all animals received systemic injections of cocaine $(15 \mathrm{mg} / \mathrm{kg}$, i.p. $)$ and 
locomotor activity was monitored for $2 \mathrm{~h}$, in $15 \mathrm{~min}$ bins, following injection.

\section{Histology}

After completion of studies that involve intracranial cannula implantation, animals were deeply anesthetized with sodium pentobarbital and were perfused by intracardiac infusion of phosphate-buffered saline $(50 \mathrm{ml})$ and $10 \%$ formaldehyde $(50$ $\mathrm{ml}$ ). Brains were stored in vials with $10 \%$ formaldehyde for at least 3 days before sectioning. Brains were sectioned $(100 \mu \mathrm{m})$ on a vibratome and sections were mounted onto gelatin-coated slides. Sections were stained with cresyl violet and dialysis probe placements were visualized by light microscopy and verified according to The Rat Brain in Stereotaxic Coordinates (Paxinos and Watson, 1997).

\section{Drugs}

Cocaine hydrochloride was purchased from Sigma Chemical Company. (St Louis, MO) LY379268 (mGluR2/3 selective agonist) and LY341495 (mGluR2/3 selective antagonist) were purchased from TOCRIS Bioscience. (Ellisville, MO) LY379268 was diluted with isotonic saline. (0.9\% sodium chloride) LY341495 was diluted with 1.2 eq. $\mathrm{NaOH}$. $\mathrm{KCl}(80 \mathrm{mM})$ buffer was prepared as followed: $\mathrm{KCl} 82.7 \mathrm{mM}, \mathrm{NaCl}$ $60 \mathrm{mM}, \mathrm{CaCl}_{2} 1.2 \mathrm{mM}, \mathrm{MgCl}_{2} 1.2 \mathrm{mM}$, plus $0.2 \mathrm{mM}$ phosphate-buffered saline to achieve a $\mathrm{pH}=7.4$. Cystine $(50 \mu \mathrm{M})$ was diluted with dialysis buffer.

\section{Statistics}

A two-way analysis of variance (ANOVA) with one repeated measure (time) was used to analyze time courses of neurochemical data. The microdialysis data were converted to percentage of baseline in order to avoid variability associated with differences in probe placement and probe recovery. Multiple comparisons were made with a modified least significant differences test (Milliken and Johnson 1984). Total motor activity data and difference in baseline neurotransmitter levels between saline and cocaine pre-treated animals were analyzed using unpaired Student's $t$-test. 


\section{Results}

Experiment 1: $m$ GluR $R_{2 / 3}$ Receptor and Mesocorticolimbic Glutamate Transmission

\section{$\underline{\text { Behavior }}$}

Figure 3-1 shows that the motor response to cocaine was significantly enhanced in animals that had previously received the daily repeated cocaine injections. After at least 1 week recovery from surgery, animals received once daily systemic injections of saline or cocaine ( $15 \mathrm{mg} / \mathrm{kg}$ ) over 4 consecutive days. After one day, one week or one month withdrawal, saline and cocaine pre-treated animals were subjected to reverse dialysis of LY341495 $(0.1,1$ and $10 \mu \mathrm{M})$ in ascending concentration into the mPFC. Within 3 days following microdialysis experiments, animals were challenged with a systemic injection of cocaine.

Effects of mGluR $_{2 / 3}$ Receptor Antagonist LY341495 on Glutamate Levels in the mPFC

Figure 3-2 shows the effects of the infusion of LY341495 $(0.1,1$ and $10 \mu \mathrm{M})$ on extracellular glutamate levels within the $\mathrm{mPFC}$ in animals that previously received repeated cocaine exposure. LY341495 produced a trend towards increased glutamate levels in saline control animals following 1 day withdrawal. This effect achieved significance in control animals following prolonged withdrawal (7 days and 30 days). After 1 day withdrawal from the repeated cocaine injections, infusions of three different doses of LY341495 into the mPFC produced significantly enhanced extracellular glutamate levels in this region in cocaine pre-treated animals as compared to saline pre-treated animals (Figure 3-2 a). However, LY341495 failed to induce an enhancement of glutamate levels in cocaine pre-treated animals as compared to saline animals following 7 days and 30 days of withdrawal (Figure 3-2b and Figure 3-2c respectively). In contrast, LY341495 produced a significant reduced glutamate level in cocaine pretreated animals following 7 days of withdrawal, when LY341495 achieved the highest concentration $(10 \mu \mathrm{M})$. Furthermore, no significant differences in basal extracellular glutamate levels ( $\mathrm{pmol} / 20 \mathrm{~min}$; mean $\pm \mathrm{SEM}$ ) were observed between saline and cocaine pre-treated animals. (a) After 1 day withdrawal: Repeated Cocaine, $3.6 \pm 0.8$; Saline, $4.6 \pm 0.6$; $(\mathrm{t}=1.048$ d.f. $=21 \mathrm{p}=0.3067)$ (b) After 7 days withdrawal: Repeated Cocaine, $5.8 \pm 1.5$; Saline, $6.6 \pm 0.8 ;(\mathrm{t}=0.4350$ d.f. $=19$ $\mathrm{p}=0.6685$ ) (c) After 30 days withdrawal: Repeated Cocaine, $8.0 \pm 1.3$; Saline, $6.2 \pm$ $0.6 ;(\mathrm{t}=1.338$ d.f. $=20 \mathrm{p}=0.1958)$. 

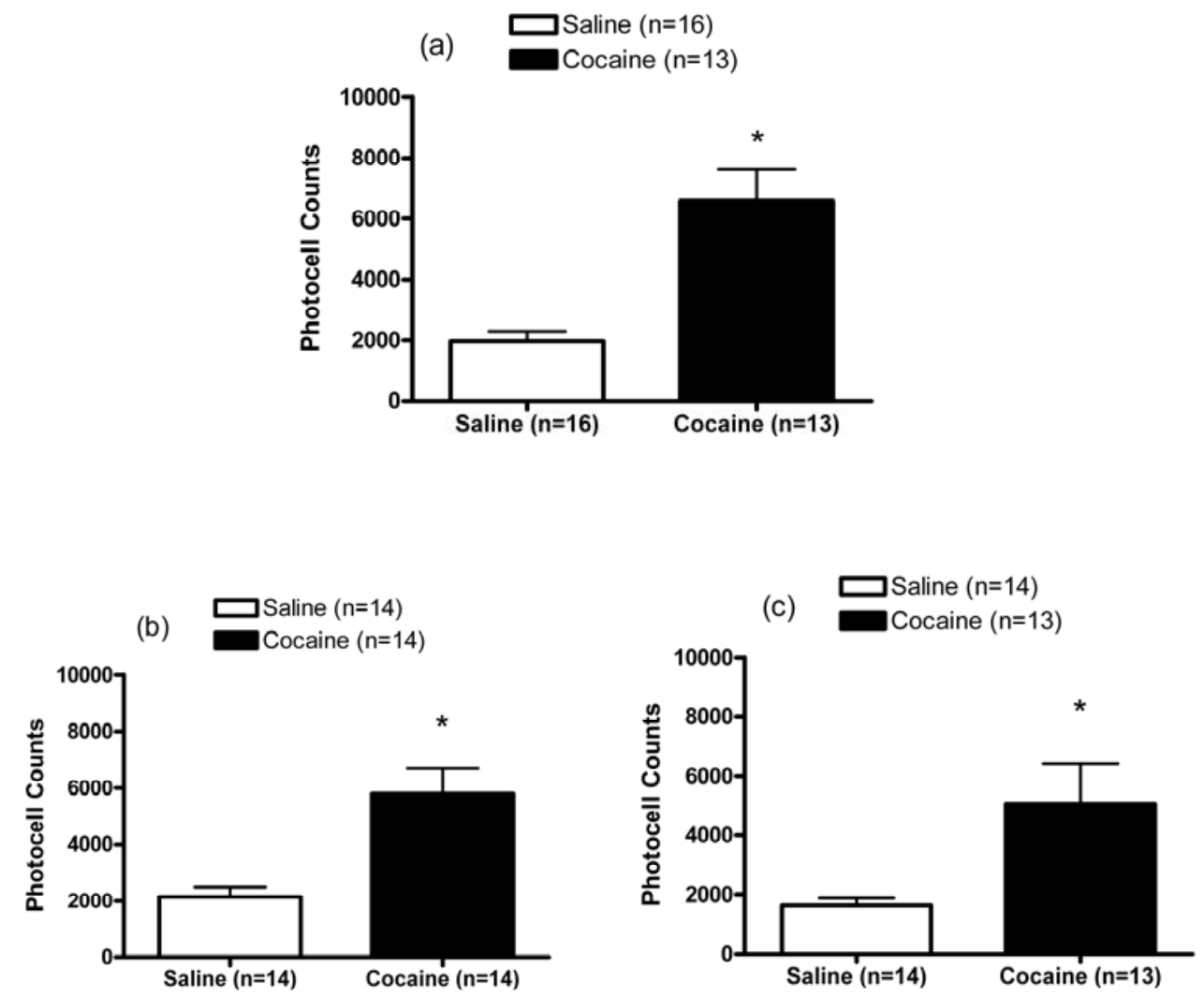

Figure 3-1. Motor stimulant response to cocaine in animals that had previously received the daily repeated cocaine injections in experiment 1. (a) 1 day withdrawal. (b) 7 day withdrawal. (c) 30 day withdrawal. Data represented as mean \pm SEM photocell counts. $* P<0.05$ compared to saline pretreatment (a) $t=4.699$ d.f. $=27$ (b) $\mathrm{t}=3.703$ d.f. $=26$ (c) $\mathrm{t}=2.573$ d.f. $=25$. 

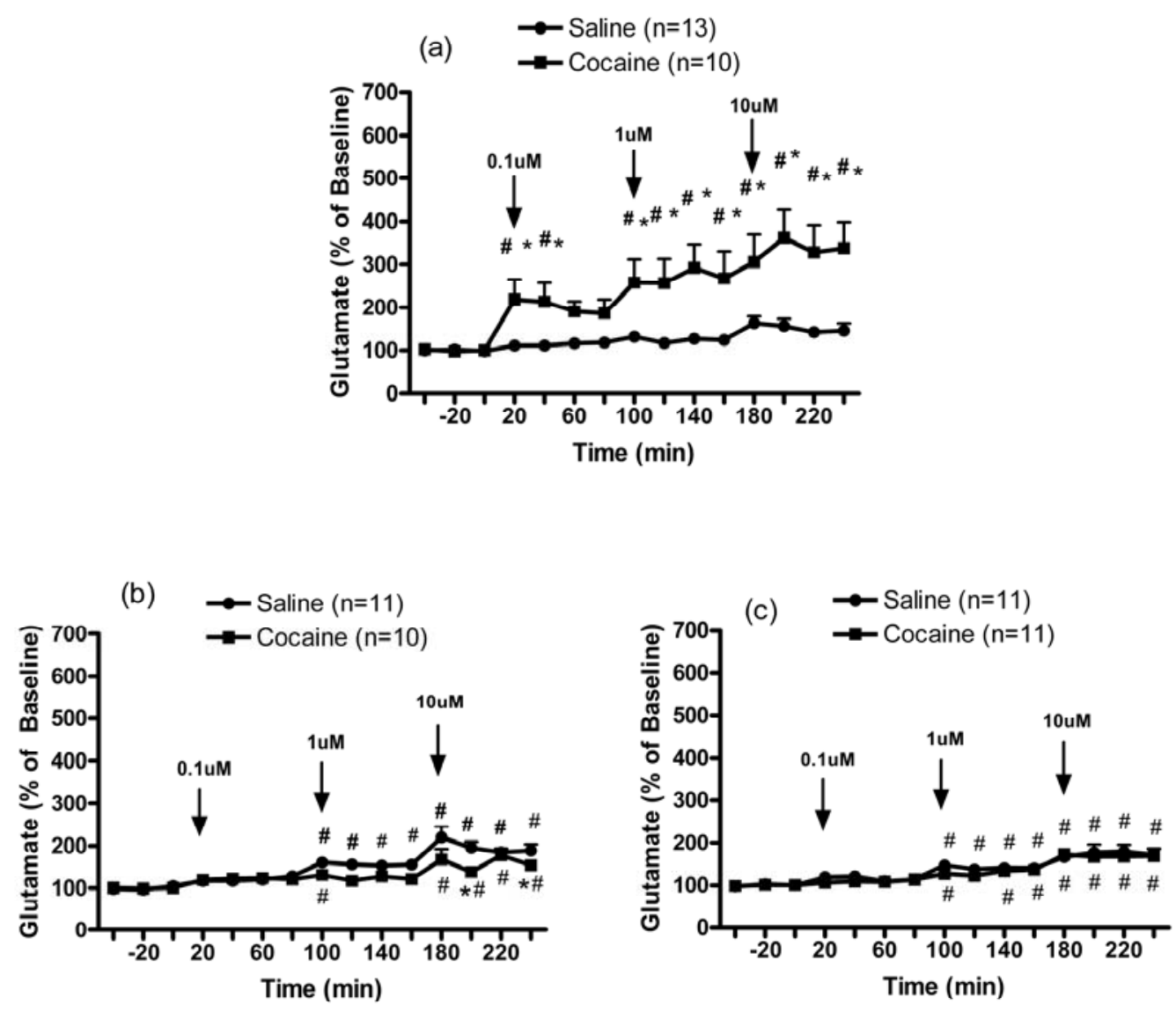

Figure 3-2. The effects of the intra-mPFC infusion of LY341495 $(0.1,1$ and $10 \mu \mathrm{M})$ on extracellular glutamate levels within the $\mathrm{MPFC}$ region withdrawn from repeated cocaine exposure. 1 day (a), 7 days (b) and 30 days (c). The significance of the differences between treatment groups was determined by two-way ANOVA with repeated-measures followed by post hoc analysis using a modified least significant differences test (Milliken and Johnson, 1984). F scores were as follows: (a) Treatment effect $\mathrm{F}(1,21)=10.078, \mathrm{p}=0.0046$; Time effect $\mathrm{F}(14,21)=12.387$, $\mathrm{p}<0.0001$; and Interaction effect $\mathrm{F}(14,294)=6.352, \mathrm{p}<0.0001$. (b) Treatment effect $\mathrm{F}(1,19)=3.748$, $\mathrm{p}=0.0679$; Time effect $\mathrm{F}(14,19)=21.419, \mathrm{p}<0.0001$; and Interaction effect $\mathrm{F}(14,266)=2.753, \mathrm{p}=0.0008$. (c) Treatment effect $\mathrm{F}(1,20)=0.8, \mathrm{p}=0.3819$; Time effect $\mathrm{F}(14,20)=25.431, \mathrm{p}<0.0001$; and Interaction effect $\mathrm{F}(14,280)=0.41, \mathrm{p}=0.9713$. $* \mathrm{P}<$ 0.05 compared to saline pre-treated animals. ${ }^{\#} \mathrm{P}<0.05$ compared to baseline. 
Effects of mGluR $2 / 3$ Receptor Antagonist LY341495 on Glutamate Levels in the NAc

The effects of the infusion of LY341495 $(0.1,1$ and $10 \mu \mathrm{M})$ into the mPFC on extracellular glutamate levels in NAc of animals that previously received repeated cocaine exposure are shown in Figure 3-3. Intra-mPFC infusions of LY341495 produced a trend towards increased of glutamate levels in the NAc of saline pre-treated animals. While this effect achieved significance in control animals following prolonged withdrawal (7 days and 30 days). Infusions of LY341495 into the mPFC produced a significant increase of extracellular glutamate concentrations in NAc of cocaine pre-treated animals after 1 day of withdrawal, compared with saline animals (Figure 3-3a). In contrast to results in mPFC shown in Figure 3-2a, this significant effect was only observed when the dose of LY341495 reached at 1 and 10 $\mu \mathrm{M}$. However, following 7 days or 30 days of withdrawal (Figure 3-3b and Figure 3-3c respectively), cocaine pre-treated animals did not show a significant increase in NAc glutamate levels following intra-mPFC infusion of LY341495 as compared with saline pre-treated animals. Also, significant differences in basal extracellular glutamate levels ( $\mathrm{pmol} / 20$ min; mean $\pm \mathrm{SEM}$ ) were not observed between saline and cocaine pre-treated animals. (a) After 1 day withdrawal: Repeated Cocaine, $3.2 \pm 0.7$; Saline, $4.7 \pm 0.8 ;(\mathrm{t}=1.396$ d.f. $=13 \mathrm{p}=0.1860)(\mathrm{b})$ After 7 days withdrawal: Repeated Cocaine, $5.3 \pm 2.3$; Saline, $2.9 \pm 0.7$; $(\mathrm{t}=1.081$ d.f. $=11 \mathrm{p}=0.3030)$ (c) After 30 days withdrawal: Repeated Cocaine, $7.2 \pm 1.8$; Saline, $4.1 \pm 1.3$; $(\mathrm{t}=1.392$ d.f. $=12$ $\mathrm{p}=0.1891)$.

\section{Effects of mGluR ${ }_{2 / 3}$ Receptor Antagonist LY341495 on Glutamate Levels in the VTA}

Figure 3-4 shows the effects of the intra-mPFC infusion of LY341495 $(0.1,1$ and $10 \mu \mathrm{M}$ ) on extracellular glutamate levels in VTA after withdrawal from the repeated cocaine injections. Similar to the results in $\mathrm{mPFC}$ and NAc, intra-mPFC infusions of LY341495 induced a trend towards increased extracellular glutamate concentrations in VTA in saline pre-treated animals following 1 day withdrawal. This effect achieved significance in control animals following prolonged withdrawal (7 days and 30 days). Intra-mPFC infusions of LY341495 produced a significant increase of glutamate levels in VTA of cocaine pre-treated animals after 1 day of withdrawal, compared with saline pre-treated animals, but the significant effect was only shown at the highest concentrations of LY341495 $(10 \mu \mathrm{M})$ (Figure 3-4a). Furthermore, cocaine pre-treated animals did not show a significant increase in VTA glutamate levels following intra-mPFC infusion of LY341495 after 7 days and 30 days of withdrawal (Figure 3-4b and Figure 3-4c respectively). Also, significant differences in basal extracellular glutamate levels ( $\mathrm{pmol} / 20$ min; mean $\pm \mathrm{SEM}$ ) were not observed between saline and cocaine pre-treated animals. (a) After 1 day withdrawal: Repeated Cocaine, $3.5 \pm 0.8$; Saline, $3.7 \pm 0.8 ;(\mathrm{t}=0.1386$ d.f. $=12 \mathrm{p}=0.8921)$ (b) After 7 days withdrawal: Repeated Cocaine, $6.3 \pm 2.2$; Saline, $4.1 \pm 1.2$; $(\mathrm{t}=0.8804$ d.f. $=13$ $\mathrm{p}=0.3946$ ). 

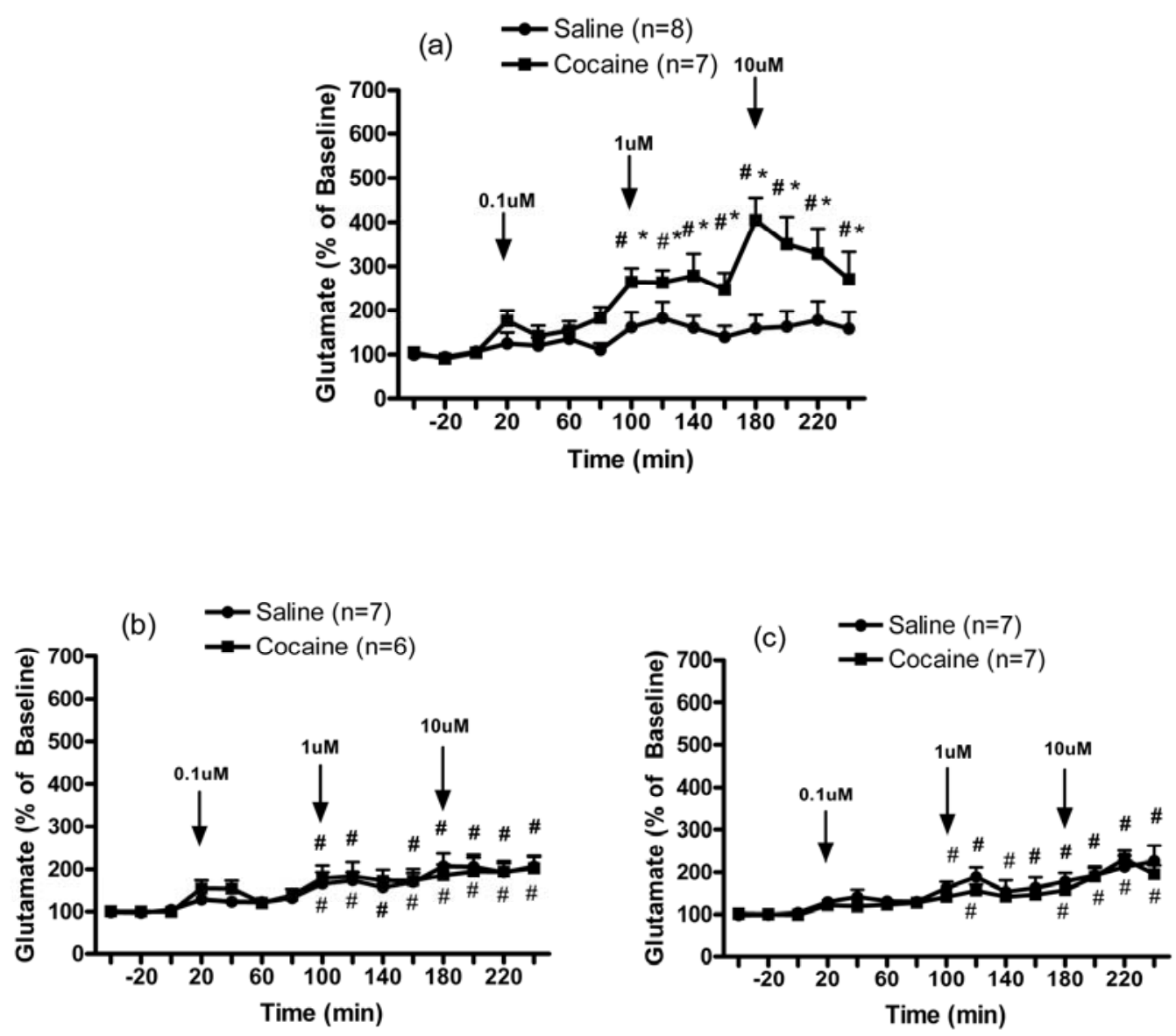

Figure 3-3. The effects of the intra-mPFC infusion of LY341495 $(0.1,1$ and $10 \mu \mathrm{M})$ on extracellular glutamate levels within the NAc region withdrawn from repeated cocaine exposure. 1 day (a), 7 days (b) and 30 days (c). The significance of the differences between treatment groups was determined by two-way ANOVA with repeated-measures followed by post hoc analysis using a modified least significant differences test (Milliken and Johnson, 1984). F scores were as follows: (a) Treatment effect $\mathrm{F}(1,12)=10.416, \mathrm{p}=0.0073$; Time effect $\mathrm{F}(14,12)=9.104$, $\mathrm{p}<0.0001$; and Interaction effect $\mathrm{F}(14,168)=3.812, \mathrm{p}<0.0001$. (b) Treatment effect $\mathrm{F}(1,11)=0.06$, $\mathrm{p}=0.811$; Time effect $\mathrm{F}(14,11)=10.719, \mathrm{p}<0.0001$; and Interaction effect $\mathrm{F}(14,154)=0.335, \mathrm{p}=0.9884$. (c) Treatment effect $\mathrm{F}(1,12)=0.756, \mathrm{p}=0.4016$; Time effect $\mathrm{F}(14,12)=14.574, \mathrm{p}<0.0001$; and Interaction effect $\mathrm{F}(14,168)=0.508, \mathrm{p}=0.9264$. ${ }^{*} \mathrm{P}<0.05$ compared to saline pre-treated animals. ${ }^{*} \mathrm{P}<0.05$ compared to baseline. 

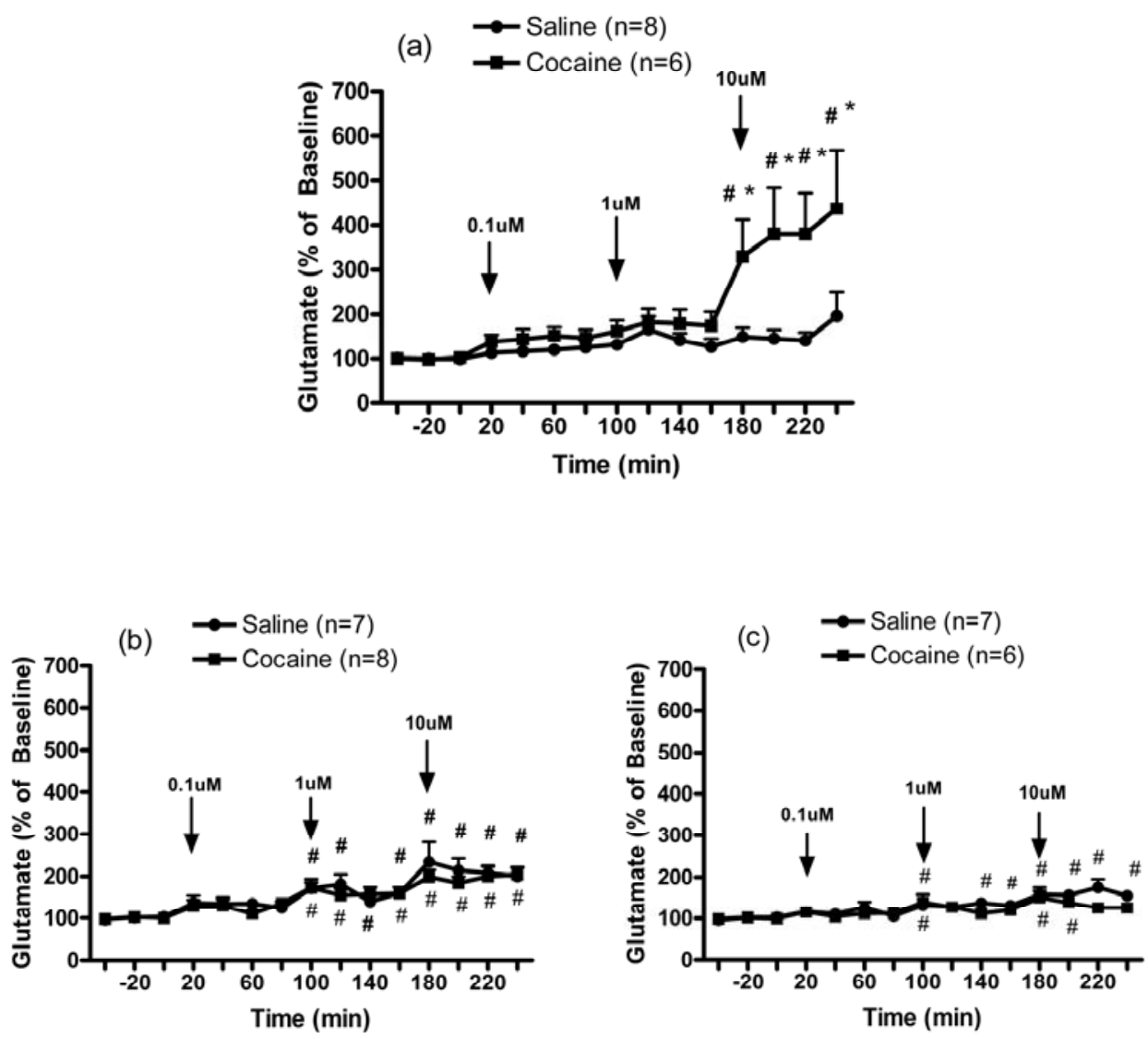

Figure 3-4. The effects of the intra-mPFC infusion of LY341495 $(0.1,1$ and $10 \mu \mathrm{M})$ on extracellular glutamate levels within the VTA region withdrawn from repeated cocaine exposure. 1 day (a), 7 days (b) and 30 days (c). The significance of the differences between treatment groups was determined by two-way ANOVA with repeated-measures followed by post hoc analysis using a modified least significant differences test (Milliken and Johnson, 1984). F scores were as follows: (a) Treatment effect $\mathrm{F}(1,12)=6.689, \mathrm{p}=0.0239$; Time effect $\mathrm{F}(14,12)=6.978, \mathrm{p}<0.0001$; and Interaction effect $F(14,168)=4.041, p<0.0001$. (b) Treatment effect $F(1,13)=0.379$, $\mathrm{p}=0.549$; Time effect $\mathrm{F}(14,13)=13.269, \mathrm{p}<0.0001$; and Interaction effect $\mathrm{F}(14,182)=0.49, \mathrm{p}=0.9363$. (c) Treatment effect $\mathrm{F}(1,11)=1.136, \mathrm{p}=0.3094$; Time effect $\mathrm{F}(14,11)=11.093, \mathrm{p}<0.0001$; and Interaction effect $\mathrm{F}(14,154)=1.842, \mathrm{p}=0.0371$. $* \mathrm{P}<$ 0.05 compared to saline pre-treated animals. ${ }^{*} \mathrm{P}<0.05$ compared to baseline. 
(c) After 30 days withdrawal: Repeated Cocaine, $6.7 \pm 1.3$; Saline, $4.4 \pm 0.6$; $(\mathrm{t}=1.599$ d.f. $=11 \mathrm{p}=0.1381$ ).

\section{Experiment 2: $m$ GluR $R_{2 / 3}$ Receptor and mPFC Glutamate Release}

\section{$\underline{\text { Behavior }}$}

After at least 1 week of recovery from surgery, animals received once daily systemic injections of saline or cocaine $(15 \mathrm{mg} / \mathrm{kg})$ over 4 consecutive days. After one day or one week withdrawal, saline and cocaine pre-treated animals were subjected to reverse dialysis with either $\mathrm{KCl}$ or cystine into the $\mathrm{mPFC}$. Studies were conducted at those time points since previous studies have shown that repeated cocaine exposure enhanced the cocaine induced glutamate overflow in the mPFC after 1 day and 7 days of withdrawal, but not 30 days of withdrawal (Williams and Steketee 2004). Within 3 days following microdialysis experiments, animals were challenged with a systemic injection of cocaine. Figure 3-5 shows that the motor stimulant response to cocaine was significantly enhanced in animals that had previously received the daily repeated cocaine injections.

Effects of GGluR $_{2 / 3}$ Receptor Agonist LY379268 on $\mathrm{K}^{ \pm}$Induced Glutamate Release in the $\mathrm{mPFC}$

Figure 3-6 shows the effects of the infusion of $\mathrm{KCl}(80 \mathrm{mM})$ into the $\mathrm{mPFC}$ on extracellular glutamate levels within this region. Infusions of $\mathrm{KCl}$ into the $\mathrm{mPFC}$ produced significantly increased extracellular glutamate concentrations in this region in saline pre-treated animals (Figure 3-6a and 6b). This effect was inhibited by intra-mPFC co-infusion of LY379268 $\left(1 \mu \mathrm{M}\right.$ ), an agonist of mGluR $_{2 / 3}$ receptor (Figure $3-6 \mathrm{a}$ and $6 \mathrm{~b})$. The $\mathrm{K}^{+}$induced glutamate release was significantly enhanced in cocaine pre-treated animals compared with saline pre-treated animals (Figure 3-6a and 6b). When $\mathrm{KCl}$ and LY379268 were co-infused into mPFC, LY379268 significantly reduced the $\mathrm{K}^{+}$induced glutamate release cocaine pre-treated animals following 1 day withdrawal (Figure 3-6a) but not 7 days withdrawal (Figure 3-6b). Moreover, no significant differences in basal extracellular glutamate levels (pmol/20 min; mean \pm SEM) were observed between saline and cocaine pre-treated animals. (a) After 1 day withdrawal: Repeated Cocaine, $4.4 \pm 0.6$; Saline, $5.7 \pm 0.7 ;(\mathrm{t}=1.409$ d.f. $=11 \mathrm{p}=0.1866)$ (b) After 7 days withdrawal: Repeated Cocaine, $3.6 \pm 0.7$; Saline, $3.0 \pm 0.9 ;(\mathrm{t}=0.5576$ d.f. $=10 \mathrm{p}=0.5894)$. 

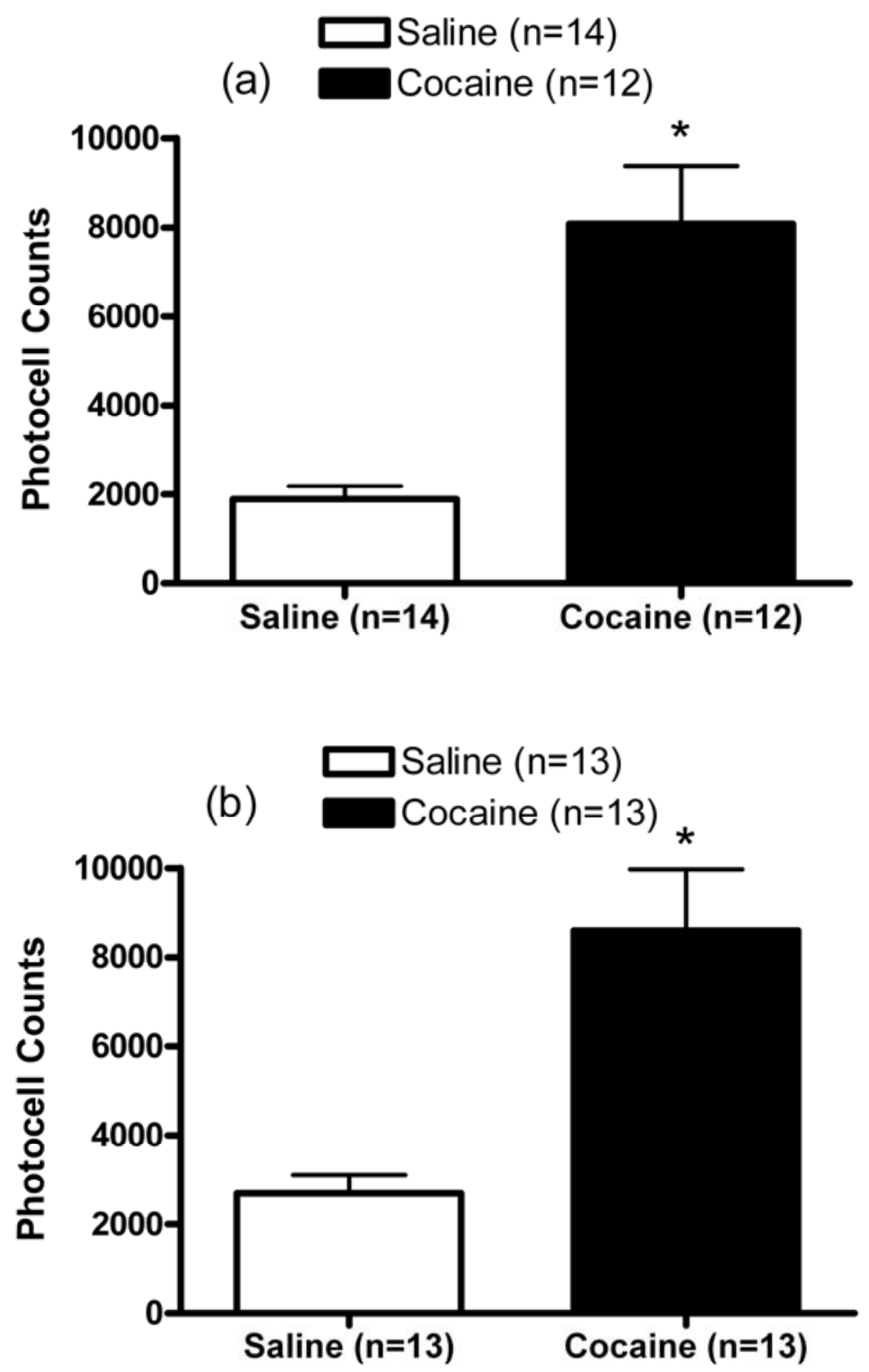

Figure 3-5. Motor stimulant response to cocaine in animals had previously received the daily repeated cocaine injections in experiment 2 . The motor stimulant response to cocaine was significantly enhanced in animals withdrawn from the daily repeated cocaine injections for 1day (a) and 7days (b) in Experiment 2 of the study of mGluR2/3 receptor and $\mathrm{mPFC}$ glutamate releasing. Data represented as mean $\pm \mathrm{SEM}$ photocell counts. ${ }^{*} P<0.05$ compared to saline pretreatment. (a) $\mathrm{t}=5.064$ d.f. $=24$ (b) $\mathrm{t}=4.206$ d.f. $=23$. 

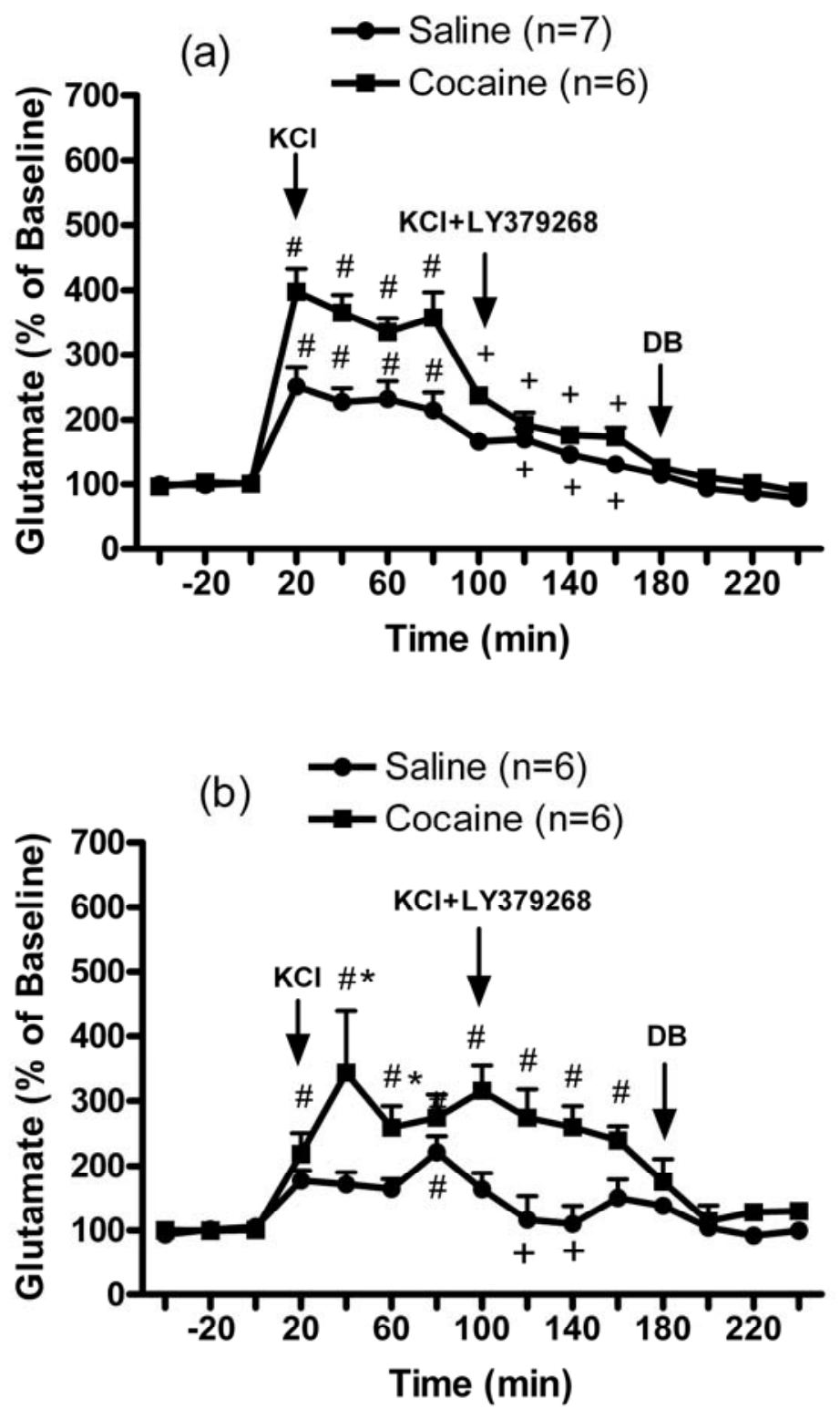

Figure 3-6. The effects of the infusion of $\mathrm{KCl}(80 \mathrm{mM})$ into the $\mathrm{mPFC}$ on extracellular glutamate levels within this region in animals withdrawn from repeated cocaine injections. 1 day (a) and 7 days (b). The significance of the differences between treatment groups was determined by two-way ANOVA with repeated-measures followed by post hoc analysis using a modified least significant differences test (Milliken and Johnson, 1984). F scores were as follows: (a) Treatment effect $\mathrm{F}(1,11)=12.581, \mathrm{p}=0.0046$; Time effect $\mathrm{F}(14,11)=68.227$, $\mathrm{p}<0.0001$; and Interaction effect $F(14,154)=7.501, p<0.0001$. (b) Treatment effect $F(1,10)=15.88$, $\mathrm{p}=0.0026$; Time effect $\mathrm{F}(14,10)=8.987, \mathrm{p}<0.0001$; and Interaction effect

$\mathrm{F}(14,140)=2.725, \mathrm{p}=0.0014$. ${ }^{*} \mathrm{P}<0.05$ compared to saline pre-treated animals. ${ }^{\#} \mathrm{P}<$ 0.05 compared with everage of baseline. ${ }^{+} \mathrm{P}<0.05$ compared with pre-treatment of LY379268. 
Effects of mGluR $2 / 3$ Receptor Aqonist LY379268 on Cystine Induced Glutamate Release in the $\mathrm{mPFC}$

Figure 3-7 shows the effects of the infusion of cystine $(50 \mu \mathrm{M})$ into the mPFC on extracellular glutamate levels within this region. Infusions of cystine into the $\mathrm{mPFC}$ produced a significant increase in extracellular glutamate concentrations in this region in saline pre-treated animals, an effect that was reduced by co-infusion of LY379268 $(1 \mu \mathrm{M})$ an agonist of $\mathrm{mGluR}_{2 / 3}$ receptor into the $\mathrm{mPFC}$ (Figure 3-7a and $7 \mathrm{~b}$ ) Cystine-induced glutamate release was significantly increased in cocaine pre-treated animals compared with saline pre-treated animals (Figure 3-7a and 7b). When cystine and LY379268, were co-infused into mPFC, LY379268 significantly reduced the cystine induced glutamate release in cocaine pre-treated animals following 1 day withdrawal (Figure 3-7a), but not 7 days withdrawal (Figure 3-7b). Moreover, significant differences in basal extracellular glutamate levels (pmol/20 min; mean \pm SEM) were not observed between saline and cocaine pre-treated animals. (a) After 1 day withdrawal: Repeated Cocaine, $5.9 \pm 0.4$; Saline, $9.1 \pm 1.9$; $(\mathrm{t}=1.509$ d.f. $=11$ $\mathrm{p}=0.1596$ ) (b) After 7 days withdrawal: Repeated Cocaine, $6.8 \pm 1.2$; Saline, $4.6 \pm 0.3$; $(\mathrm{t}=1.797$ d.f. $=12 \mathrm{p}=0.0975)$.

\section{$\underline{\text { Histology }}$}

Figure 3-8 shows a representative photo-micrograph of dialysis probe sites in the $\mathrm{mPFC}, \mathrm{NAc}$ and VTA. Dialysis probes were located medial to the forceps minor corpus callosum (fmi) with the dialysis membrane encompassing the infralimbic and prelimbic subregions of the mPFC (a). In the NAc, the probe placements were medial to the anterior commissure (ac) with the majority of the membrane located in the core of the nucleus accumbens (Figure 3-8b). The probes in VTA were in the region of the paranigral nuclei $(\mathrm{PN})(\mathrm{c})$. Animals with incorrect dialysis probe placements were removed from the study.

\section{Discussion}

The present study demonstrated that intra-mPFC infusions of LY341495, an antagonist of $\mathrm{mGluR}_{2 / 3}$ receptors increased glutamate levels in mesocorticolimbic brain regions including $\mathrm{mPFC}, \mathrm{NAc}$ and VTA. Results from the present study suggested that the $\mathrm{mPFC} \mathrm{mGluR}_{2 / 3}$ receptor plays an inhibitory role in modulating the mesocorticolimbic glutamate transmission. LY341495 enhanced the glutamate levels in these brain regions in cocaine sensitized animals after 1 day of withdrawal. However, it was not capable of enhancing glutamate transmission within these regions in animals withdrawn from repeated cocaine injections for a relatively long period ( 7 days and 30 days). These results therefore suggested that the inhibitory tone by $\mathrm{mPFC}$ $\mathrm{mGluR}_{2 / 3}$ receptor was transiently increased following a short term of cocaine 

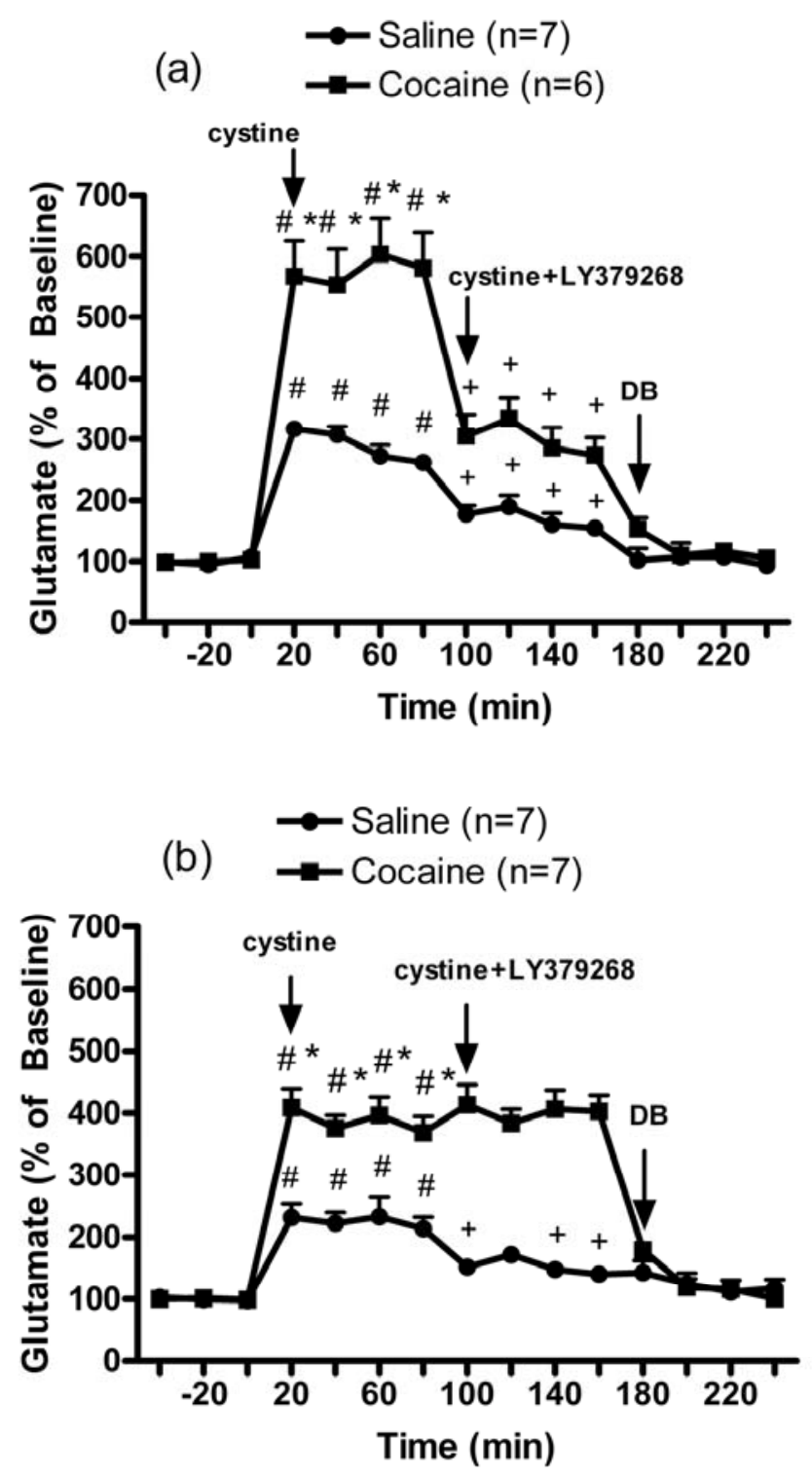

Figure 3-7. The effects of the infusion of cystine $(50 \mu \mathrm{M})$ into the $\mathrm{mPFC}$ on extracellular glutamate levels within this region in animals withdrawn from repeated cocaine injections. 1 day (a) and 7 days (b). The significance of the differences between treatment groups was determined by two-way ANOVA with repeated-measures followed by post hoc analysis using a modified least significant differences test (Milliken and Johnson, 1984). F scores were as follows: (a) Treatment effect $\mathrm{F}(1,11)=25.504, \mathrm{p}=0.0004$; Time effect $\mathrm{F}(14,11)=103.994, \mathrm{p}<0.0001$; and Interaction effect $F(14,154)=20.471, p<0.0001$. (b) Treatment effect $F(1,12)=51.326$, $\mathrm{p}<0.0001$; Time effect $\mathrm{F}(14,12)=69.644, \mathrm{p}<0.0001$; and Interaction effect $\mathrm{F}(14,168)=24.636, \mathrm{p}<0.0001 .{ }^{*} \mathrm{P}<0.05$ compared to saline pre-treated animals. ${ }^{\#} \mathrm{P}<$ 0.05 compared with everage of baseline. ${ }^{+} \mathrm{P}<0.05$ compared with pre-treatment of LY379268. 

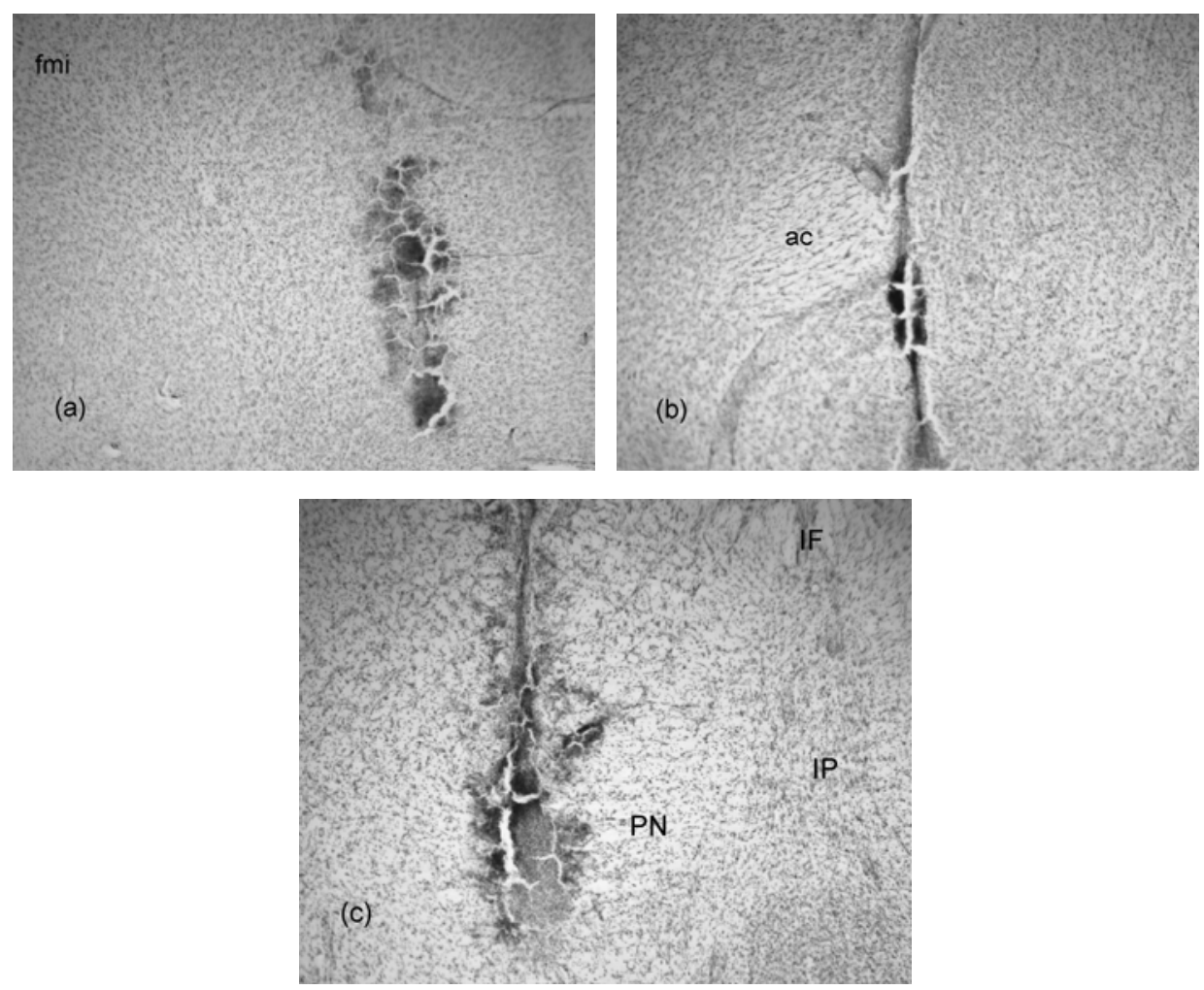

Figure 3-8. Representative micrographs of dialysis probe sites in the mPFC, NAc and VTA. Dialysis probes were located medial to the forceps minor corpus callosum (fmi) with the dialysis membrane encompassing the infralimbic and prelimbic subregions of the mPFC (a). In the NAc, the probe placements were medial to the anterior commissure (ac) with the majority of the membrane located in the core of the nucleus accumbens (b). The probes in VTA were in the region of the paranigral nuclei (PN) (c). 
withdrawal, but no longer enhanced following prolonged withdrawal. Further results in the second experiment of the present study demonstrated that repeated cocaine exposure enhanced the vesicular glutamate release $\left(\mathrm{K}^{+}\right.$induced glutamate release) and non-vesicular glutamate release (cystine induced glutamate release) in the mPFC. It was shown that $\mathrm{mPFC} \mathrm{mGluR}_{2 / 3}$ receptor was able to inhibit the vesicular glutamate release and non-vesicular glutamate release in animals withdrawn from repeated cocaine injections after 1 day but not 7 days. Generally, the functional reduction of $\mathrm{mPFC} \mathrm{mGluR}_{2 / 3}$ receptor caused loss of inhibitory control of the excitatory drive within $\mathrm{mPFC}$ and thereafter at least partly resulted in the enhanced excitatory outputs from mPFC to other subcortical regions including NAc and VTA.

Previous studies have shown that infusion of $\mathrm{mGluR}_{2 / 3}$ antagonist into $\mathrm{mPFC}$ elevated the glutamate levels, while agonist did not alter the glutamate levels (Melendez and Kalivas 2003), suggesting that there is an inhibitory tone regulating mPFC glutamate levels. Therefore, LY341495, an antagonist of GluR $_{2 / 3}$ was chosen for the present study. It was expected that functional reduction of $\mathrm{mGluR}_{2 / 3}$ receptor caused by repeated cocaine exposure could remove this inhibitory tone. However, the results from the present study actually showed that the inhibitory tone via $\mathrm{mGluR}_{2 / 3}$ receptor was transiently enhanced. This is supported by the results showing that intra-mPFC infusions of LY341495 generated an enhanced glutamate level in mPFC of animals that received repeated cocaine exposure following 1 day of withdrawal. It is unclear what mechanisms underlie the transiently increased inhibitory tone provided by $\mathrm{mGluR}_{2 / 3}$ receptors. One of the possible explanations is that the transient increase in inhibitory tone was a result of cocaine-mediated increases in $\mathrm{mPFC}$ glutamate transmission. Nonetheless, our results do demonstrate that the transiently increased inhibitory tone via mGluR $_{2 / 3}$ receptor was absent with long term expression of cocaine sensitization. Following 7 days but not 30 days of withdrawal, LY341495 produced a significant derease in glutamate level in the $\mathrm{mPFC}$ of cocaine pre-treated animals. These data suggested the inhibitory tone on $\mathrm{mGluR}_{2 / 3}$ receptor was reduced. This result is correlated with additional findings that the $\mathrm{mGluR}_{2 / 3}$ receptor lost its ability to inhibit vesicular and non-vesicular release of glutamate in cocaine pre-treated animals following 7 days of withdrawal. This reduction of $\mathrm{mGluR}_{2 / 3}$ inhibitory tone could be due to the functional reduction of $\mathrm{mGluR}_{2 / 3}$ receptor. To clarify this issue, an experiment using $\mathrm{mGluR}_{2 / 3}$ receptor agonists might be able to demonstrate whether repeated cocaine exposure reduces the ability of $\mathrm{mPFC}$ $\mathrm{mGluR}_{2 / 3}$ receptor to inhibit the mesocorticloimbic glutamate transmission.

It is well known that glutamatergic transmission from mPFC to NAc and VTA is an important component in the expression of cocaine sensitization (Wolf 1998; Kalivas 2004). The results from the present study demonstrated that repeated cocaine exposure can alter mesocorticolimbic glutamate transmission at least partly by $\mathrm{mPFC}$ $\mathrm{mGluR}_{2 / 3}$ receptor. However, the effects of this alteration via acting on $\mathrm{mPFC}$ $\mathrm{mGluR}_{2 / 3}$ receptors on specific glutamate transmission pathways within mesocorticolimbic dopamine system appear to be different. This is supported by the 
results from the present study that intra-mPFC infusions of $\mathrm{mGluR}_{2 / 3}$ receptor antagonist LY341495 at the highest concentration produced enhanced glutamate levels in the VTA of cocaine sensitized animals shown in Figure 3-4a, but the enhanced glutamate levels in the NAc was initially induced at an intermediate concentration of LY341495. This can be explained by the difference in the neuronal circuitry. For example, the glutamatergic projections from the mPFC to the VTA can be both direct and indirect via the pedunculopontine tegmentum (Sesack et al. 1989; Hurley et al. 1991).It is well known that extracellular glutamate within mPFC comes from glutamatergic nerve terminals via vesicular release as well as from glial cells via cystine/glutamate antiporter (Melendez et al. 2005). It has been suggested that extracellular concentrations of glutamate measured via microdialysis actually reflect both of these sources of glutamate (Baker et al. 2002). Thus, we were prompted to examine whether repeated cocaine exposure produces specific alterations in the modulation of extracellular glutamate sources within the mPFC. One of the alterations demonstrated in the present study is the functional reduction of $\mathrm{mPFC} \mathrm{mGluR}_{2 / 3}$ receptor. Our results clearly showed that repeated cocaine exposure reduced the ability of $\mathrm{mGluR}_{2 / 3}$ receptor to inhibit the vesicular glutamate release $\left(\mathrm{K}^{+}\right.$induced glutamate release) and non-vesicular glutamate release (cystine induced glutamate release). Interestingly, our data also indicated that repeated cocaine exposure enhanced the ability of $\mathrm{K}^{+}$and cystine to increase glutamate levels in the mPFC (Figure 3-6 and 3-7).

In accordance with these findings, $\mathrm{K}^{+}$induced glutamate release has been shown to be increased by repeated amphetamine exposure (Stephans and Yamamoto 1995). Thus, the enhanced ability of cystine to increase glutamate levels in the mPFC of cocaine sensitized animals could also be due to the overall enhanced glutamate releasability and hyperactivity of the pyramidal neuron that have recurrent collaterals to form a positive feedback within the MPFC. Although, it been shown in a previous study that repeated cocaine treatment reduced activity of the cystine/glutamate antiporter in the NAc (Baker et al. 2003), comparison of the results from Figure 3-7a and $3-7 \mathrm{~b}$ suggested that the ability of mPFC cystine/glutamate antiporter appears to be intact following a long term withdrawal due to a similar percentage of enhancement.

$\mathrm{mGluR}_{2}$ and $\mathrm{mGluR}_{3}$ are found in various combinations of presynaptic, postsynaptic and glial localizations that may reflect differential modulation of excitatory amino acid transmission (Petralia et al. 1996; Conn and Pin 1997). Although, the present study did not demonstrate a role of specifically localized $\mathrm{mGluR}_{2 / 3}$ receptor in the development of cocaine sensitization, previous studies may provide some clues. For example, the functional reduction of $\mathrm{mGluR}_{2 / 3}$ receptor, acting as autoreceptor in NAc, has been shown in animals that received repeated cocaine pretreatment (Xi et al. 2002). A more recent study demonstrated that the mGluR $_{2 / 3}$ receptor induced postsynaptic LTD was impaired in mPFC pyramidal neurons after repeated cocaine exposure (Huang et al. 2007). And our present study also showed the functional reduction of $\mathrm{mPFC} \mathrm{mGluR}_{2 / 3}$ receptor that can inhibit the 
cystine/glutamate antiporter, which is mainly localized on glia cells. Taken together, it

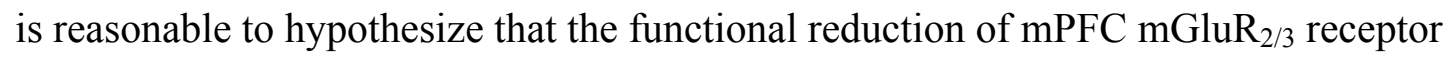
is "universal" within this brain region.

There are not many studies that have been done to demonstrate the mechanisms underlying the functional reduction of $\mathrm{mPFC} \mathrm{mGluR}_{2 / 3}$ receptor. Recently, expression of AGS3 protein was found to be enhanced in the mPFC of cocaine-sensitized animals (Bowers et al. 2004). AGS3, an activator of $G$ protein signaling has been shown to interact with Gi $\alpha$ subunits and disrupt $\mathrm{G}$ protein signaling by interfering with receptor coupling (De Vries et al. 2000; Natochin et al. 2000) Hence, this would prevent actions of receptors that couple to Gi proteins, such as $\mathrm{mGluR}_{2 / 3}$ receptors. It should be noted that previous studies have also suggested that repeated cocaine exposure can reduce the function of other Gi protein coupled receptors, including $\mathrm{D}_{2}$ and $\mathrm{GABA}_{\mathrm{B}}$ receptors (Beyer and Steketee 2002; Steketee and Beyer 2005). It has been found that repeated cocaine exposure decreased $\mathrm{G}$ protein levels such as Gi and Go but had no effect on the levels of Gs alpha and $\mathrm{G}$ beta in other subcortical regions including NAc and VTA (Nestler et al. 1990). Therefore, disruption of Gi protein coupling via increased AGS3 expression will possibly enhance Gs protein coupling to some extent, which may partly be one of the reasons that repeated cocaine exposure enhanced the cAMP signaling pathways (Nestler et al. 2001). Therefore, the possible balance for Gi vs Gs coupled receptor signaling might be an important component in modulation of $\mathrm{mPFC}$ excitatory transmission in the development of cocaine sensitization.

In conclusion, the present study demonstrated that long term expression of sensitization to cocaine is associated with an altered modulation of the

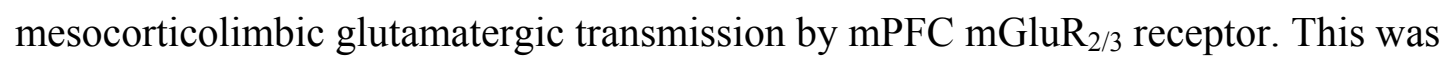
supported by the results that intra-mPFC infusions of GGluR $_{2 / 3}$ antagonist LY341495 transiently produced a significant enhancement in glutamatergic transmission within mesocorticolimbic brain regions, including mPFC, NAc and VTA. This effect is associated with an enhancement of vesicular and non-vesicular release of glutamate in the $\mathrm{mPFC}$. Functional reduction of $\mathrm{mGluR}_{2 / 3}$ receptor may in part underlie the mechanisms increasing the vesicular and non-vesicular release of glutamate following prolonged withdrawal from repeated cocaine exposre. To our knowledge, our study firstly demonstrated that repeated cocaine exposure altered the modulations of releasable sources of glutamate within the $\mathrm{mPFC}$, which provide the excitatory drive for the glutamatergic outputs from the mPFC. Thus, the present results support the hypotheses that functional reduction of $\mathrm{mGluR}_{2 / 3}$ receptor regulation of excitatory transmission from the mPFC to the VTA and NAc may play an important role in the development of sensitization to cocaine. 


\section{Chapter 4. General Discussion}

\section{Data Summary}

\section{Results in Chapter 2}

The initial studies were designed to determine the effects of intra-mPFC injections of the $\mathrm{mGluR}_{2 / 3}$ agonist APDC on cocaine-induced motor activity and on the development of sensitization to cocaine. First, we examined the dose-response effects of injection of APDC $(0.015-15 \mathrm{nmol} / \mathrm{side})$ into the $\mathrm{mPFC}$ on the acute motor-stimulant response to cocaine $(15 \mathrm{mg} / \mathrm{kg}$, i.p.). Intra-mPFC APDC dose-dependently reduced acute cocaine-induced motor activity. The lowest dose in the present studies that significantly reduced the acute motor-stimulant response to cocaine was $1.5 \mathrm{nmol} / \mathrm{side}$. Additionally, the effects of APDC were prevented by co-injections of intra-mPFC LY341495 (1.5 nmol/side), an mGluR $_{2 / 3}$ antagonist. Additional studies demonstrated that intra-mPFC microinjection of APDC also reduced the initiation of behavioral and neurochemical sensitization to cocaine. Once sensitization was induced, however, intracortical administration of APDC did not block the cocaine-induced behavioral and neurochemical responses in sensitized animals after 7 days and 30 days withdrawal. In contrast, intra-mPFC injections of APDC were found to block the expression of behavioral and neurochemical sensitization in sensitized animals after 1 day withdrawal.

In conclusion, the data in Chapter 2 demonstrated that repeated intra-mPFC APDC prevented cocaine-induced behavioral sensitization. However the ability of intra-mPFC APDC to block the cocaine induced behavioral response was lost in sensitized animals after more prolonged withdrawal. The data provide additional support for the idea that sensitization to cocaine results from an increase in excitatory output from the mPFC to subcortical regions, which in part results from the disinhibition of mPFC pyramidal neurons. (Steketee 2005) This conclusion is supported by recent findings which demonstrated that repeated cocaine exposure reduces $\mathrm{mGluR}_{2 / 3}$ responsiveness at $\mathrm{mPFC}$ excitatory synapses after 5 days withdrawal. (Huang et al. 2007) The molecular and cellular mechanisms underlying the loss of receptor function still remains to be clarified. Findings on the mechanism will be helpful to generate new treatment targets for cocaine addiction.

\section{Results in Chapter 3}

The present study demonstrated that intra-mPFC infusions of LY341495, an antagonist of $\mathrm{mGluR}_{2 / 3}$ receptor increased glutamate levels in the mesocorticolimbic brain regions including mPFC, NAc and VTA, which are well known brain regions involved in cocaine sensitization. Results from the present study suggested that $\mathrm{mPFC}$ 
$\mathrm{mGluR}_{2 / 3}$ receptor plays an inhibitory role in modulating the mesocorticolimbic glutamate transmission. Nonetheless, this effect was enhanced in animals following 1 day withdrawal but not 7 days and 30 days withdrawn from repeated cocaine injections. These results therefore suggested that the transiently increased inhibitory tone of $\mathrm{mPFC} \mathrm{mGluR}_{2 / 3}$ receptor was no longer apparent following a long term withdrawal from cocaine. This effect could be due to the functional reduction of $\mathrm{mPFC} \mathrm{mGluR}_{2 / 3}$ receptor or decreased inhibitory tone. To clarify this issue, experiments using $\mathrm{mGluR}_{2 / 3}$ receptor agonist could examine whether the agonist could reduce mesocorticolimbic glutamate transmission and whether its ability would be reduced following a prolonged cocaine withdrawal. Additional studies showed that enhanced vesicular ( $\mathrm{K}^{+}$evoked) and non-vesicular (cystine evoked) glutamate release in the mPFC of cocaine sensitized animals. Furthermore, results in the present study also demonstrated that repeated cocaine exposure altered the modulation of the releasable pools of glutamate via $\mathrm{mPFC} \mathrm{mGluR}_{2 / 3}$ receptor within this brain region. It was shown in the present study that $\mathrm{mPFC} \mathrm{mGluR}_{2 / 3}$ receptor lost its ability to inhibit the vesicular glutamate release $\left(\mathrm{K}^{+}\right.$induced glutamate release $)$and non-vesicular glutamate release (cystine induced glutamate release) in animals withdrawn from repeated cocaine injections after 7 days but not 1 day. Generally, the functional reduction of $\mathrm{mPFC} \mathrm{mGluR}_{2 / 3}$ receptor caused loss of inhibitory control of glutamate release within $\mathrm{mPFC}$ and thereafter at least partly resulted in the enhanced excitatory drive, which in turn generated increased excitatory outputs from $\mathrm{mPFC}$ to other subcortical regions including NAc and VTA.

In conclusion, repeated cocaine exposure alters the modulation of the mesocorticolimbic glutamatergic transmission by $\mathrm{mPFC} \mathrm{mGluR}_{2 / 3}$ receptor. This was supported by the results that intra-mPFC infusions of GGluR $_{2 / 3}$ antagonist LY341495 produced a significant, transient enhancement in glutamatergic transmission within mesocorticolimbic brain regions, including mPFC, NAc and VTA following a short term of cocaine withdrawal. This effect was no longer apparent following more prolonged withdrawal. Moreover, our study showed enhanced vesicular ( $\mathrm{K}^{+}$evoked) and non-vesicular (cystine evoked) glutamate release in cocaine sensitized animals. Repeated cocaine exposure altered the modulations of releasable pools of glutamate within the $\mathrm{mPFC}$ by $\mathrm{mGluR}_{2 / 3}$ receptor. Thus, the present results support the hypotheses that repeated cocaine exposure alters the mesocorticolimbic glutamate transmission by medial prefrontal cortex $\mathrm{mGluR}_{2 / 3}$ receptor.

\section{Potential Mechanisms Underlying the Alterations of Medial Prefrontal Excitatory Transmission by Repeated Cocaine Exposure}

Recently, several research groups have focused on the role of mesocorticolimbic glutamate transmission in drug dependence. The excitatory output from the mPFC to other brain regions has been demonstrated as an important component associated with psychomotor stimulant mediated behaviors (Wolf 1998; Tzschentke 2000; Kalivas 
2004; Steketee 2005). Consistent with previous findings, the present studies demonstrated that pharmacological manipulation of mPFC neurotransmission via activation of Group II mGluRs had a great impact on the development of cocaine sensitization. Moreover, initial studies suggested that long term expression of cocaine sensitization is correlated with the loss of ability of mPFC Group II mGluRs to modulate behavioral and neurochemical responses to cocaine. Similar results that demonstrated that dopamine $\mathrm{D}_{2}$ receptor and $\mathrm{GABA}_{\mathrm{B}}$ receptor function were reduced following prolonged cocaine withdrawal have been reported by our group. It should be noted that while these Gi protein coupled receptors may function in different neurotransmitter system, they generally can play an inhibitory role in modulating the mPFC excitatory output. Therefore, combined with previous studies, the current studies support our hypotheses that sensitization to cocaine results from an increase in excitatory output from the $\mathrm{mPFC}$ to subcortical regions, which in part results from the disinhibition of mPFC pyramidal neurons. Furthermore, we were prompted to examine the modulations of mesocortiolimbic glutamate transmission by repeated cocaine exposure. What we found was that repeated cocaine exposure transiently increased the inhibitory tone maintained via $\mathrm{mGluR}_{2 / 3}$ receptor following a short term withdrawal. These results may help to explain why intra-mPFC injections of $\mathrm{mGluR}_{2 / 3}$ receptor agonist, APDC prevent the short term expression of cocaine sensitization but not the long term expression. However, this inhibitory tone was back to normal (similar to saline pre-treated animals) following prolonged withdrawal. Furthermore, our data showed that repeated cocaine exposure enhanced the vesicular and non-vesicular release of glutamate in the mPFC. This modulation, however, is altered in part by $\mathrm{mGluR}_{2 / 3}$ receptor following prolonged withdrawal, suggesting loss of inhibitory control over mPFC glutamate release. Therefore, the potential mechanisms underlying the alterations of medial prefrontal excitatory transmission by repeated cocaine exposure will be discussed in the followings.

\section{Medial Prefrontal Extracellular Glutamate Level}

Medial prefrontal cortical glutamate release provides the excitatory drive for the outputs from the mPFC. Various factors, such as neurotransmitters and/or their receptors are potentially involved in modulation of $\mathrm{mPFC}$ glutamate release. Thus, studies have been largely focused on how repeated cocaine exposure alters the modulations of these factors. Previous studies demonstrated that repeated methamphetamine exposure can increase neuronal glutamate release (Stephans and Yamamoto 1995), which is supported by the current findings that $\mathrm{K}^{+}$induced $\mathrm{mPFC}$ glutamate release was increased following repeated cocaine exposure shown in Chapter 3. Glutamate terminals exist on projections mainly from the amygdala, hippocampus, and mediodorsal nucleus of the thalamus, as well as axon collaterals of pyramidal neurons within mPFC (Sesack et al. 1989). It has been shown that repeated cocaine administration increases membrane excitability of pyramidal neurons in the rat medial prefrontal cortex (Nasif et al. 2005). Therefore, increased excitability of 
mPFC pyramidal neurons could result in enhanced glutamate releases via axon collaterals within the $\mathrm{mPFC}$ region. Besides ionotropic glutamate receptors, metabotropic glutamate receptors have been suggested to play a potential role in regulating glutamate release (Conn and Pin 1997; Melendez and Kalivas 2003; Melendez et al. 2005). Therefore, functional alterations of these receptors could also play a potential role underlying the enhanced glutamate release induced by repeated cocaine exposure. Currently, there are eight mGluRs, classified into three groups of receptors based on sequence homology, preferred signal transduction pathway and pharmacology. Group I ( $\left.\mathrm{mGluR}_{1 / 5}\right)$ can enhance phosphoinositide hydrolysis. Group II ( mGluR $\left._{2 / 3}\right)$ and Group III ( mGluR $\left._{4 / 6 / 7 / 8}\right)$ both can inhibit the adenylyl cyclase-mediated formation of cAMP (Conn and Pin 1997; Kenny and Markou 2004). Within cortex, there are many subtypes of mGluRs, however, the Group I and II receptors are abundant in the mPFC area (Watkins 2000).

A previous study demonstrated that reverse dialysis of the group I agonist (RS)-3,5-dihydroxyphenylglycine (DHPG) into the NAc resulted in an increase in extracellular glutamate levels. However, the capacity of DHPG to induce glutamate release was markedly reduced following 3 weeks withdrawal from cocaine. Similarly, locomotor activity induced by intra-NAc microinjection of DHPG was significantly blunted 3 weeks after repeated cocaine administration. This diminished function is associated with decreased levels of $\mathrm{mGluR}_{5}$ and Homer $1 \mathrm{~b} / \mathrm{c}$ protein (Swanson et al. 2001). One possible mechanism underlying the functional reduction of Group I mGluRs is a reduction in Homerlb/c protein that is of importance in regulating the signaling through Group I mGluRs (Kane et al. 2005; Mao et al. 2005; Tappe and Kuner 2006). Also the Group I mGluRs induced PKC phosphorylation of GluR2 parallels downregulation of AMPA receptors (Nakazawa et al. 1997). Thus, reduced levels of Group I mGluRs may facilitate the sensitization of AMPA-induced behaviors produced by repeated cocaine administration (Pierce et al. 1996). Taken together, these results indicated that functional reduction of Group I mGluRs in the NAc is associated with the long term expression of cocaine sensitization. However, a recent study found that pre-treatment of Group I mGluR antagonists prevented the expression of behavioral sensitization to cocaine (Dravolina et al. 2006). Thereby, it suggested that effects of repeated cocaine on the alteration of Group I mGluRs may be different in specific brain regions. This effect might not be due to the antagonism on Group I mGluRs in the NAc, since functional reduction of Group I mGluRs in the NAc is associated with the long term expression of cocaine sensitization. In fact, intra-mPFC infusion of Group I mGluR agonist was found to elevate the glutamate level in this region (Melendez and Kalivas 2003). Thus, Group I mGluRs in the mPFC might be involved in regulation of expression of cocaine sensitization. Unfortunately, few studies have been done to examine the effects of repeated cocaine exposure on the modulations of mesocorticolimbic glutamate transmission by medial prefrontal Group I mGluRs.

Group II mGluRs are also important in regulating glutamate levels in the mPFC. 
$\mathrm{mGluR}_{2 / 3}$ receptors were indicated to play an inhibitory role in regulating glutamate release (Conn and Pin 1997; Kenny and Markou 2004), which was also shown in the present study. mGluR $_{2 / 3}$ receptors can also be localized postsynaptically. Postsynaptic $\mathrm{mGluR}_{2 / 3}$ receptors in rat prefrontal cortex can induce LTD through postsynaptic PKC activation and IP3 receptor-mediated postsynaptic increases of $\mathrm{Ca}^{2+}$ concentration via phospholipase $\mathrm{C}$ and probably also phospholipase D (Otani et al. 2002). Also $\mathrm{mGluR}_{2 / 3}$ receptors can be localized on glia cells, where they can inhibit the activity of cystine/glutamate exchanger (Baker et al. 2002). Importantly, GluR $_{2 / 3}$ receptors can also function as presynaptic heteroceptors on monoaminergic terminals in the $\mathrm{mPFC}$ or as autoreceptors inhibiting the presynaptic glutamate release (Cartmell and Schoepp 2000; Xi et al. 2002). $\mathrm{mGluR}_{2}$ and $\mathrm{mGluR}_{3}$ are found in various combinations of presynaptic, postsynaptic and glial localizations that may reflect differential modulation of excitatory amino acid transmission (Conn and Pin 1997; Kenny and Markou 2004). Although, the present studies did not demonstrate the role of specifically localized $\mathrm{mGluR}_{2 / 3}$ receptor in the development of cocaine sensitization, previous studies may provide some clues. For example, the functional reduction of $\mathrm{mGluR}_{2 / 3}$ receptor, acting as autoreceptor in the NAc has been shown in animals that received repeated cocaine pretreatment (Xi et al. 2002). A more recent study demonstrated that the $\mathrm{mGluR}_{2 / 3}$ receptor induced postsynaptic LTD was impaired in $\mathrm{mPFC}$ pyramidal neurons after repeated cocaine exposure (Huang et al. 2007). Similar with previous studies (Baker et al. 2002), our present study also showed the loss of ability of $\mathrm{mPFC} \mathrm{mGluR}_{2 / 3}$ receptor to inhibit the cystine/glutamate antiporter. Taken together, it is reasonable to hypothesize that the functional reduction of $\mathrm{mPFC} \mathrm{mGluR}_{2 / 3}$ receptor is "universal" within this brain region. One of the possible mechanisms underlying the functional reduction of $\mathrm{mGluR}_{2 / 3}$ receptor was the increased levels of the activators of G protein signaling-3 (AGS3) (Bowers et al. 2004), which could diminishe prefrontal cortical receptor signaling that is coupled to inhibitory $\mathrm{G}$ proteins, including $\mathrm{mGluR}_{2 / 3}$ receptor. Another research group recently suggested a new model (Huang et al. 2007). Repeated cocaine administration increases levels of dopamine (DA) in the mPFC that leads to increased formation of cAMP via the activation of $\mathrm{D}_{1}$ receptors. Extracellular cAMP is then metabolized to adenosine by phosphodiesterase and ecto5'-nucleotidase and acts on the adenosine $\mathrm{A}_{3}$ receptors. The activation of adenosine $\mathrm{A}_{3}$ receptors would increase PKC activity and thereby trigger an impairment of group II mGluR function, resulting in an impairment of LTD induced by DCG-IV or LY379268. Although the mechanisms described above have differences, the joint point in these mechanisms is the enhanced cAMP signaling. Decreased Gi $\alpha$ signaling may promote signaling through other $\mathrm{G}$ proteins, such as Gs $\alpha$. For example, Gs $\alpha$-coupled $\mathrm{D}_{1}$ dopamine receptors have been shown to increase membrane excitability in a PKA-dependent manner in pyramidal neurons of the PFC (Wang and O'Donnell 2001). Thus, the mechanisms discussed above are consistent with the critical role of dopamine $\mathrm{D}_{1}$ receptors in the PFC in cocaine-induced drug seeking and conditioned place preference (Capriles et al. 2003; Sanchez et al. 2003).

One thing that should be discussed here is that a functional reduction of $\mathrm{mGluR}_{2 / 3}$ 
receptor was suggested to be correlated with the long term expression of cocaine sensitization in studies shown in Chapter 2, but studies in Chapter 3 actually demonstrated a transient enhancement of inhibitory tone via $\mathrm{mGluR}_{2 / 3}$ following a short term withdrawal from cocaine. Moreover, this transient increase in inhibitory tone was no longer apparent following long term withdrawal. We explained that this transient increase in inhibitory tone could be a compensatory response to cocaine-mediated increases in mPFC glutamate transmission, while the loss of this transient increase in inhibitory tone could be due to either the reduction of $\mathrm{mGluR}_{2 / 3}$ receptor signaling or simply the reduction of the inhibitory tone. The basal glutamate levels can influence the inhibitory tone of $\mathrm{mGluR}_{2 / 3}$ receptor. However, basal glutamate levels in the $\mathrm{mPFC}$ appear to be intact after repeated cocaine exposure (Williams and Steketee 2004). But, we cannot rule out other possibilities such as repeated cocaine may increase the glutamate reuptake rate to allow less glutamate to diffuse to the location of $\mathrm{mGluR}_{2 / 3}$ receptor. Generally, to clarify the issues here, $\mathrm{mGluR}_{2 / 3}$ receptor agonist could be used to demonstrate whether repeated cocaine exposure can reduce the ability of the agonist to modulate the glutamate transmission within the mesocorticolimbic brain regions.

Results in Chapter 3 suggested that cocaine influences a non-neuronal pool of glutamate regulated by the cystine/glutamate antiporter. As shown in Chapter 3, repeated cocaine exposure enhanced the cystine induced glutamate release in the mPFC. However, the ability of $\mathrm{mGluR}_{2 / 3}$ receptor agonist, LY379268 to inhibit this non-neuronal glutamate release was no longer apparent following a prolonged cocaine withdrawal. These results indicate the dis-inhibition of the non-neuronal glutamate release induced by repeated cocaine exposure may partly contribute to enhanced glutamate release seeing during early sensitization. However, the enhanced non-neuronal glutamate release induced by repeated cocaine exposure following a short term withdrawal is not correlated with the loss of inhibition of $\mathrm{mGluR}_{2 / 3}$ receptor. Thus, mGluR $_{2 / 3}$ receptor agonist, LY379268 still can inhibit the cystine induced glutamate release. These results further suggested other mechanisms many control the activity of cystine/glutamate antiporter. Studies have demonstrated that the activity of cystine/glutamate antiporter is regulated by cAMP-dependent protein kinase and PKA stimulated activity of the antiporter (Tang and Kalivas 2003). Moreover, in contrast to previous studies that repeated cocaine exposure has been shown to reduce the activity of this antiporter in the NAc (Baker et al. 2003), the present studies shown in Chapter 3 suggested that reduction of the activity of this antiporter might not happen in the mPFC following a long term cocaine withdrawal. It has been shown that this antiporter plays an important role in maintaining endogenous tone on $\mathrm{mGluR}_{2 / 3}$ receptor in the NAc (Baker et al. 2002). Probably, the reduction of activity of this antiporter may only help to reduce the inhibitory tone via $\mathrm{mGluR}_{2 / 3}$ receptor so as to enhance the synaptic glutamate release. While inhibitors of this antiporter did not alter the basal glutamate level in the mPFC (Melendez et al. 2005), suggesting that there are may be other mechanisms underlying maintaining endogenous tone on $\mathrm{mGluR}_{2 / 3}$ receptor in the $\mathrm{mPFC}$. 
The dynamic glutamate level in the mPFC is not only influenced by the glutamate release, but also regulated by the glutamate synthesis and clearance. Glutamine is a common precursor for the biosynthesis of both glutamate and GABA. Glutamine can be transported in and out of neurons and astrocytes utilizing different glutamine carriers. Three such carriers have recently been cloned and characterized, referred to as ASCT2, GlnT and SN1. They are differentially expressed in brain cells; ASCT2 and SN1 being astrocytic and GlnT being neuronal (Schoepfer et al. 1994; Hediger and Welbourne 1999). They play different roles in glutamine influx and efflux and hence control the availability of glutamine. The neurotransmitter glutamate can be mainly synthesized from glutamine by the action of phosphate-activated glutaminase (Hediger and Welbourne 1999). Then, cytoplasmic glutamate is transported into vesicles by the vesicular glutamate transporter. After synaptic release, glutamate is taken up by excitatory amino acid transporters (EAATs) into either nerve terminals or glia cells (Bridges and Esslinger 2005). Following entry into the nerve terminal, glutamate is pumped into synaptic vesicles by a vesicular transport system. Three different vesicular glutamate transporter proteins (VGLUT1-3) representing unique markers for glutamatergic neurons were recently characterized (Hisano 2003; Shigeri et al. 2004). Glutamate metabolism, which to a large extent takes place in glial cells, is catalyzed either by glutamine synthetase or glutamate dehydrogenase (Bridges and Esslinger 2005). Probably because cocaine is the blocker of monoamine transporter, previous studies have focused on the effects of chronic cocaine on the monoamine transporter, such as DAT, NET and SERT as well as the vesicular monoamine transporter: VMAT. Although some studies reported the alterations of these transporters following chronic cocaine exposure with regard to either function or expression (Wilson and Kish 1996), no agreement has been achieved. Therefore, studies on glutamate transporters have not been conducted, although it has been reported that chronic amphetamine treatment increased tissue levels of glutamate in frontal cortex (Kim et al. 1981) and decreased aspartate turnover was found in the frontal cortex of cocaine self-administering rats compared to yoked-vehicle controls, but not yoked-cocaine rats (Smith et al. 2003). Few studies have been done to demonstrate the effects of chronic psychomotor stimulants, such as cocaine on the key proteins regulating glutamate biosynthesis and clearance. However, a recent study has shown that the colocalization of postsynaptic glutamate transporters and mGluRs limits mGluR-mediated signals and mGluR-dependent forms of plasticity by up-taking glutamate in cerebellar slices (Brasnjo and Otis 2001). Thus, it is possible that chronic psychomotor stimulants exposure could alter the glutamate receptor signaling by affecting the EAATs.

\section{Medial Prefrontal Glutamate Outputs and Mesolimbic Dopamine System}

When combined with previous research (Steketee 2005), the studies shown in Chapter 2 demonstrated that pharmacological manipulation of the mPFC could alter the development of cocaine sensitization. Cocaine induced behavioral sensitization is 
well correlated with the augmented cocaine-mediated dopamine response in the NAc. This is the reason we monitored dopamine levels in the NAc in the studies in Chapter 2 , and defined it as the neurochemical sensitization. Since enhanced glutamate output is associated with the augmented behavioral response and neurochemical response to cocaine challenge, it seems necessary to discuss the glutamate output in regulating the mesolimbic dopamine system in the development of sensitization.

It has been reported that systemic cocaine injection $(15 \mathrm{mg} / \mathrm{kg})$ or intra-VTA administration of the $\mathrm{D}_{1}$ receptor agonist SKF-82958, but not the $\mathrm{D}_{2}$ receptor agonist quinpirole increased glutamate efflux in the VTA of naive rats (Kalivas and Duffy 1995). It also has been demonstrated that PFC neurons projecting to VTA express mRNA for $D_{1}$ receptors (Lu et al. 1997). Therefore it has been proposed that psychomotor stimulant, such as cocaine augments somatodendritic DA efflux, which increases the $\mathrm{D}_{1}$ receptor-mediated stimulation of glutamate release from the glutamatergic terminals projecting from PFC to VTA. Therefore, it is hypothesized that repeated elevation of VTA glutamate levels, caused by repeated cocaine injections, leads to adaptive changes in the VTA that contribute to the induction and maintenance of sensitization (Kalivas 1995). This hypothesis might support that repeated intra-mPFC injections of $\mathrm{mGluR}_{2 / 3}$ receptor agonist, APDC might inhibit the pyramidal neurons and decrease the repeated elevation of VTA glutamate levels so as to prevent the initiation of cocaine sensitization. By using extracellular single unit recording and microiontophoresis, it has been shown that the responsiveness of VTA DA neurons to the excitatory effects of glutamate is significantly enhanced in cocaine-pretreated animals (White et al. 1995), an effect associated with increased sensitivity of AMPA receptors (Zhang et al. 1997). By using the AMPA/NMDA ratio as a marker for LTP, LTP in VTA dopamine neurons has been reported (Ungless et al. 2001; Saal et al. 2003). Furthermore, it has been found that the AMPA/NMDA ratio was increased in brain slices prepared one day after a single injection of amphetamine, cocaine, morphine, ethanol, or nicotine (Saal et al. 2003). Thus, there is a growing evidence that the initiation of sensitization involves LTP at excitatory synapses onto VTA dopamine neurons (Wolf 1998).

It has been reported that a challenge injection of $15 \mathrm{mg} / \mathrm{kg}$ cocaine, given 3 weeks after discontinuing 1 week of daily cocaine injections $(30 \mathrm{mg} / \mathrm{kg})$, elicited increased glutamate efflux in core but not shell of the NAc in cocaine sensitized animals (Pierce et al. 1996). The results suggested increased glutamate efflux in the NAc is associated with expression of cocaine sensitization. In addition, the augmented DA efflux in the NAc is correlated with the expression of cocaine sensitization. Although it is not clear which neurotransmitter system change is secondary to the other, it seems that both systems are involved in the modulation of medium spiny NAc neurons following repeated cocaine exposure. Anatomical studies have shown that medium spiny NAc neurons receive inputs from DA terminals from VTA as well as cortical terminals (Sesack and Pickel 1992). Basically dopamine inhibits the medium spiny NAc neurons by acting on the dopamine receptors such as $\mathrm{D}_{1}$ receptor, while glutamate can 
excite these neurons by acting on glutamate neurons such as AMPA receptor. It has been found that GluR1, GluR2, and NR1 mRNA levels and immunoreactivity in the NAc were decreased following a long term withdrawal from amphetamine (Lu et al. 1997; Lu and Wolf 1999). It has also been shown that $\mathrm{D}_{1}$ receptor exhibits supersensitivity after repeated cocaine or amphetamine exposure (Henry and White 1991). Therefore, these changes would result in more efficient inhibition of medium spiny neurons. Since inhibition of medium spiny neurons in the NAc has been associated with enhanced locomotor activity (Pennartz et al. 1994), both the reduced glutamate response and enhanced dopamine inhibition may contribute synergistically to the expression of behavioral sensitization. However, some studies have shown an increase in GluR1 and NR1 immunoreactivity in the NAc 3 weeks after discontinuation of repeated cocaine (Pierce et al. 1996). These results suggest increased AMPA receptor responsiveness in cocaine sensitized rats. One possible explanation is that the increased AMPA receptor expression may be mainly on dopamine terminals, which would enhance dopamine release in the NAc. Group II mGluRs may also play a role in negatively regulating dopamine release in the NAc. $\mathrm{KCl}$ stimulated release of dopamine from the slices of the rat NAc was reduced by $\mathrm{mGluR}_{2 / 3}$ receptor agonist (Chaki et al. 2006). An $\mathrm{mGluR}_{2 / 3}$ receptor antagonist, MGS0039, increases extracellular dopamine levels in the NAc shell (Karasawa et al. 2006). Thus, repeated cocaine exposure induced functional reduction of $\mathrm{mGluR}_{2 / 3}$ receptor in the NAc (Xi et al. 2002) might contribute to the enhanced dopamine release in the NAc of cocaine sensitized animals. However, whether physiological levels of glutamate in the NAc can increase the release of DA by acting on presynaptic glutamate receptors located on dopaminergic terminals is still in debate, since it has been reported that DA release could be induced by a relatively high dose of glutamate or glutamate receptor agonists (Svensson et al. 1994), which did not represent physiological levels of glutamate in the NAc. Probably, the regulation of dopamine release by glutamate in the NAc may become efficient only in conditions such as enhanced glutamate transmission in cocaine sensitized animals. This could be partly explained by the anatomical data, which have shown that the terminals of mPFC efferents in the NAc are often in close apposition with dopaminergic terminals, but they do not form direct synapses with dopaminergic terminals (Sesack and Pickel 1992). Therefore, high glutamate level may be needed to allow glutamate to diffuse to the dopaminergic terminals and activate the glutamate receptors.

Generally, although glutamate may directly increase dopamine release from the dopaminergic terminals in the NAc, the enhanced dopamine release associated with sensitization seems mainly due to the enhanced dopamine neuron activity in the VTA. The enhanced VTA dopamine neuron activity could be maintained through the LTP on the excitatory synapse, which was described above. Moreover, it has been found that the ratio of AMPA/NMDA receptor-mediated EPSCs was decreased at synapses made by prefrontal cortical afferents onto medium spiny neurons in the shell of the NAc (Thomas et al. 2001) This is supported by previous studies that have shown that all drugs of abuse decrease the firing of NAc neurons (Wise 1998). Thus, the 
GABAergic inputs into the VTA dopamine neurons could be reduced. Therefore, disinhibition of VTA dopamine neurons by the long term depression of excitatory synaptic transmission on medium spiny neurons (MSN) in the NAc could be possible. This could be supported by a recent finding that repeated cocaine exposure increased the probability of spike initiation facilitating the LTP in VTA dopamine neuron by reduced the amplitude of GABA-mediated synaptic currents (Liu et al. 2005).

\section{Conclusions}

Given the involvement of glutamate circuits in reward-related brain regions and evidence of cocaine-induced glutamatergic dysregulation, as a glutamate rich brain region, $\mathrm{mPFC}$ serves as a very important glutamate output source, which is a critical component in drug induced brain disorders, including withdrawal, relapse and craving. The current studies have attempted to identify some of the factors that are involved in the development of cocaine sensitization, which is thought to play an important role in craving and relapse in drug addiction. The actions of mPFC Group II mGluRs demonstrated in this project include modulation of glutamate release from vesicular and non-vesicular pools within this brain region, modulation of the mesocorticolimbic glutamate transmission and influence on the dopamine response in the NAc. In addition, the role of Group II mGluRs in the development of cocaine sensitization could be more complicated due to the potential loss of the fuctionality of this receptor. The principal finding from the current project was that the development of cocaine sensitization is associated with the alteration of mesocorticolimbic glutamate transmission by Group II mGluRs. Generally, these findings support the hypothesis that sensitization results, in part, from a decrease in inhibitory modulation of pyramidal neurons, allowing for an increase in excitatory transmission in the mesocorticolimbic brain regions. 


\section{LIST OF REFERENCES}

Ackerman J. M. and White F. J. (1990) A10 somatodendritic dopamine autoreceptor sensitivity following withdrawal from repeated cocaine treatment. Neurosci Lett 117, 181-187.

Adewale A. S., Platt D. M. and Spealman R. D. (2006) Pharmacological stimulation of group ii metabotropic glutamate receptors reduces cocaine self-administration and cocaine-induced reinstatement of drug seeking in squirrel monkeys. $J$ Pharmacol Exp Ther 318, 922-931.

al-Tikriti M. S., Roth R. H., Kessler R. M. and Innis R. B. (1992) Autoradiographic localization of dopamine D1 and D2 receptors in rat cerebral cortex following unilateral neurotoxic lesions. Brain Res 575, 39-46.

Aldrich M. and Barker R. (1976) Historical aspects of cocaine use and abuse, in Cocaine: chemical, biological,clinical, social and treatment aspects (Mule S ed) pp 3-11, CRC Press, Brooklyn.

Bacon S. J., Headlam A. J., Gabbott P. L. and Smith A. D. (1996) Amygdala input to medial prefrontal cortex (mPFC) in the rat: a light and electron microscope study. Brain Res 720, 211-219.

Badiani A., Anagnostaras S. G. and Robinson T. E. (1995) The development of sensitization to the psychomotor stimulant effects of amphetamine is enhanced in a novel environment. Psychopharmacology (Berl) 117, 443-452.

Badiani A., Oates M. M. and Robinson T. E. (2000) Modulation of morphine sensitization in the rat by contextual stimuli. Psychopharmacology (Berl) 151, 273-282.

Baker D. A., McFarland K., Lake R. W., Shen H., Tang X. C., Toda S. and Kalivas P. W. (2003) Neuroadaptations in cystine-glutamate exchange underlie cocaine relapse. Nat Neurosci 6, 743-749.

Baker D. A., Shen H. and Kalivas P. W. (2002) Cystine/glutamate exchange serves as the source for extracellular glutamate: modifications by repeated cocaine administration. Amino Acids 23, 161-162.

Baker D. A., Xi Z. X., Shen H., Swanson C. J. and Kalivas P. W. (2002) The origin and neuronal function of in vivo nonsynaptic glutamate. J Neurosci 22, 9134-9141.

Bardo M. T. (1998) Neuropharmacological mechanisms of drug reward: beyond dopamine in the nucleus accumbens. Crit Rev Neurobiol 12, 37-67.

Bardo M. T. and Bevins R. A. (2000) Conditioned place preference: what does it add to our preclinical understanding of drug reward? Psychopharmacology (Berl) 153, 31-43.

Bardo M. T., Rowlett J. K. and Harris M. J. (1995) Conditioned place preference using opiate and stimulant drugs: a meta-analysis. Neurosci Biobehav Rev 19, 39-51.

Beitner-Johnson D., Guitart X. and Nestler E. J. (1991) Dopaminergic brain reward regions of Lewis and Fischer rats display different levels of tyrosine hydroxylase and other morphine- and cocaine-regulated phosphoproteins. Brain Res 561, 
147-150.

Bell K. and Kalivas P. W. (1996) Context-specific cross-sensitization between systemic cocaine and intra-accumbens AMPA infusion in the rat. Psychopharmacology (Berl) 127, 377-383.

Beyer C. E., Stafford D., LeSage M. G., Glowa J. R. and Steketee J. D. (2001) Repeated exposure to inhaled toluene induces behavioral and neurochemical cross-sensitization to cocaine in rats. Psychopharmacology (Berl) 154, 198-204.

Beyer C. E. and Steketee J. D. (2000) Intra-medial prefrontal cortex injection of quinpirole, but not SKF 38393, blocks the acute motor-stimulant response to cocaine in the rat. Psychopharmacology (Berl) 151, 211-218.

Beyer C. E. and Steketee J. D. (2002) Cocaine sensitization: modulation by dopamine D2 receptors. Cereb Cortex 12, 526-535.

Borgland S. L., Taha S. A., Sarti F., Fields H. L. and Bonci A. (2006) Orexin A in the VTA is critical for the induction of synaptic plasticity and behavioral sensitization to cocaine. Neuron 49, 589-601.

Boudreau A. C. and Wolf M. E. (2005) Behavioral sensitization to cocaine is associated with increased AMPA receptor surface expression in the nucleus accumbens. J Neurosci 25, 9144-9151.

Bowers M. S., McFarland K., Lake R. W., Peterson Y. K., Lapish C. C., Gregory M. L., Lanier S. M. and Kalivas P. W. (2004) Activator of G protein signaling 3: a gatekeeper of cocaine sensitization and drug seeking. Neuron 42, 269-281.

Bradberry C. W. (2007) Cocaine sensitization and dopamine mediation of cue effects in rodents, monkeys, and humans: areas of agreement, disagreement, and implications for addiction. Psychopharmacology (Berl) 191, 705-717.

Brasnjo G. and Otis T. S. (2001) Neuronal glutamate transporters control activation of postsynaptic metabotropic glutamate receptors and influence cerebellar long-term depression. Neuron 31, 607-616.

Bridges R. J. and Esslinger C. S. (2005) The excitatory amino acid transporters: pharmacological insights on substrate and inhibitor specificity of the EAAT subtypes. Pharmacol Ther 107, 271-285.

Capriles N., Rodaros D., Sorge R. E. and Stewart J. (2003) A role for the prefrontal cortex in stress- and cocaine-induced reinstatement of cocaine seeking in rats. Psychopharmacology (Berl) 168, 66-74.

Carey R. J., DePalma G., Damianopoulos E. and Shanahan A. (2005) Stimulus gated cocaine sensitization: interoceptive drug cue control of cocaine locomotor sensitization. Pharmacol Biochem Behav 82, 353-360.

Carlezon W. A., Jr. and Nestler E. J. (2002) Elevated levels of GluR1 in the midbrain: a trigger for sensitization to drugs of abuse? Trends Neurosci 25, 610-615.

Carlezon W. A., Jr., Thome J., Olson V. G., Lane-Ladd S. B., Brodkin E. S., Hiroi N., Duman R. S., Neve R. L. and Nestler E. J. (1998) Regulation of cocaine reward by CREB. Science 282, 2272-2275.

Carr D. B. and Sesack S. R. (2000) GABA-containing neurons in the rat ventral tegmental area project to the prefrontal cortex. Synapse 38, 114-123.

Cartmell J. and Schoepp D. D. (2000) Regulation of neurotransmitter release by 
metabotropic glutamate receptors. J Neurochem 75, 889-907.

Castner S. A. and Goldman-Rakic P. S. (1999) Long-lasting psychotomimetic consequences of repeated low-dose amphetamine exposure in rhesus monkeys. Neuropsychopharmacology 20, 10-28.

Chaki S., Yoshikawa R. and Okuyama S. (2006) Group II metabotropic glutamate receptor-mediated regulation of dopamine release from slices of rat nucleus accumbens. Neurosci Lett 404, 182-186.

Chefer V. I., Moron J. A., Hope B., Rea W. and Shippenberg T. S. (2000) Kappa-opioid receptor activation prevents alterations in mesocortical dopamine neurotransmission that occur during abstinence from cocaine. Neuroscience 101, 619-627.

Chefer V. I. and Shippenberg T. S. (2002) Changes in basal and cocaine-evoked extracellular dopamine uptake and release in the rat nucleus accumbens during early abstinence from cocaine: quantitative determination under transient conditions. Neuroscience 112, 907-919.

Chi H., Jang J. K., Kim J. H. and Vezina P. (2006) Blockade of group II metabotropic glutamate receptors in the nucleus accumbens produces hyperlocomotion in rats previously exposed to amphetamine. Neuropharmacology 51, 986-992.

Churchill L., Swanson C. J., Urbina M. and Kalivas P. W. (1999) Repeated cocaine alters glutamate receptor subunit levels in the nucleus accumbens and ventral tegmental area of rats that develop behavioral sensitization. J Neurochem 72, 2397-2403.

Collins S. L. and Izenwasser S. (2004) Chronic nicotine differentially alters cocaine-induced locomotor activity in adolescent vs. adult male and female rats. Neuropharmacology 46, 349-362.

Conde F., Audinat E., Maire-Lepoivre E. and Crepel F. (1990) Afferent connections of the medial frontal cortex of the rat. A study using retrograde transport of fluorescent dyes. I. Thalamic afferents. Brain Res Bull 24, 341-354.

Conde F., Maire-Lepoivre E., Audinat E. and Crepel F. (1995) Afferent connections of the medial frontal cortex of the rat. II. Cortical and subcortical afferents. J Comp Neurol 352, 567-593.

Conn P. J. and Pin J. P. (1997) Pharmacology and functions of metabotropic glutamate receptors. Annu Rev Pharmacol Toxicol 37, 205-237.

Cornish J. L. and Kalivas P. W. (2001) Cocaine sensitization and craving: differing roles for dopamine and glutamate in the nucleus accumbens. $J$ Addict Dis 20, 43-54.

Cowan R. L., Sesack S. R., Van Bockstaele E. J., Branchereau P., Chain J. and Pickel V. M. (1994) Analysis of synaptic inputs and targets of physiologically characterized neurons in rat frontal cortex: combined in vivo intracellular recording and immunolabeling. Synapse 17, 101-114.

Creese I. and Iversen S. D. (1973) Blockage of amphetamine induced motor stimulation and stereotypy in the adult rat following neonatal treatment with 6-hydroxydopamine. Brain Res 55, 369-382.

Crombag H. S., Badiani A., Maren S. and Robinson T. E. (2000) The role of 
contextual versus discrete drug-associated cues in promoting the induction of psychomotor sensitization to intravenous amphetamine. Behav Brain Res 116, 1-22. Cryan J. F., Hoyer D. and Markou A. (2003) Withdrawal from chronic amphetamine induces depressive-like behavioral effects in rodents. Biol Psychiatry 54, 49-58.

Cunningham C. L., Niehus J. S. and Noble D. (1993) Species difference in sensitivity to ethanol's hedonic effects. Alcohol 10, 97-102.

De Vries L., Fischer T., Tronchere H., Brothers G. M., Strockbine B., Siderovski D. P. and Farquhar M. G. (2000) Activator of G protein signaling 3 is a guanine dissociation inhibitor for Galpha i subunits. Proc Natl Acad Sci U S A 97, 14364-14369.

Dearry A., Gingrich J. A., Falardeau P., Fremeau R. T., Jr., Bates M. D. and Caron M. G. (1990) Molecular cloning and expression of the gene for a human D1 dopamine receptor. Nature 347, 72-76.

Dingledine R., Borges K., Bowie D. and Traynelis S. F. (1999) The glutamate receptor ion channels. Pharmacol Rev 51, 7-61.

Doherty M. D. and Gratton A. (1999) Effects of medial prefrontal cortical injections of GABA receptor agonists and antagonists on the local and nucleus accumbens dopamine responses to stress. Synapse 32, 288-300.

Dong Y., Cooper D., Nasif F., Hu X. T. and White F. J. (2004) Dopamine modulates inwardly rectifying potassium currents in medial prefrontal cortex pyramidal neurons. J Neurosci 24, 3077-3085.

Dong Y., Nasif F. J., Tsui J. J., Ju W. Y., Cooper D. C., Hu X. T., Malenka R. C. and White F. J. (2005) Cocaine-induced plasticity of intrinsic membrane properties in prefrontal cortex pyramidal neurons: adaptations in potassium currents. $J$ Neurosci 25, 936-940.

Downs A. W. and Eddy N. B. (1932) The effect of repeated doses of cocaine on the rat. J Pharmacol Exp Ther 46, 199-200.

Dravolina O. A., Danysz W. and Bespalov A. Y. (2006) Effects of group I metabotropic glutamate receptor antagonists on the behavioral sensitization to motor effects of cocaine in rats. Psychopharmacology (Berl) 187, 397-404.

Ehrman R. N., Robbins S. J., Childress A. R. and O'Brien C. P. (1992) Conditioned responses to cocaine-related stimuli in cocaine abuse patients.

Psychopharmacology (Berl) 107, 523-529.

Epping-Jordan M. P., Watkins S. S., Koob G. F. and Markou A. (1998) Dramatic decreases in brain reward function during nicotine withdrawal. Nature 393, 76-79.

Esposito R. U., Porrino L. J., Seeger T. F., Crane A. M., Everist H. D. and Pert A. (1984) Changes in local cerebral glucose utilization during rewarding brain stimulation. Proc Natl Acad Sci U S A 81, 635-639.

Everitt B. J. and Wolf M. E. (2002) Psychomotor stimulant addiction: a neural systems perspective. $J$ Neurosci 22, 3312-3320.

Fitzgerald L. W., Ortiz J., Hamedani A. G. and Nestler E. J. (1996) Drugs of abuse and stress increase the expression of GluR1 and NMDAR1 glutamate receptor subunits in the rat ventral tegmental area: common adaptations among cross-sensitizing agents. J Neurosci 16, 274-282. 
Foltin R. W. and Fischman M. W. (1992) Self-administration of cocaine by humans: choice between smoked and intravenous cocaine. J Pharmacol Exp Ther 261, 841-849.

Font L., Aragon C. M. and Miquel M. (2006) Ethanol-induced conditioned place preference, but not aversion, is blocked by treatment with D-penicillamine, an inactivation agent for acetaldehyde. Psychopharmacology (Berl) 184, 56-64.

Gao W. Y., Lee T. H., King G. R. and Ellinwood E. H. (1998) Alterations in baseline activity and quinpirole sensitivity in putative dopamine neurons in the substantia nigra and ventral tegmental area after withdrawal from cocaine pretreatment. Neuropsychopharmacology 18, 222-232.

Gerber U., Gee C. E. and Benquet P. (2007) Metabotropic glutamate receptors: intracellular signaling pathways. Curr Opin Pharmacol 7, 56-61.

Goldman-Rakic P. S., Leranth C., Williams S. M., Mons N. and Geffard M. (1989) Dopamine synaptic complex with pyramidal neurons in primate cerebral cortex. Proc Natl Acad Sci U S A 86, 9015-9019.

Goldstein A. (1994) Addiction: from biology to drug policy, New York: WH Freeman.

Grobin A. C. and Deutch A. Y. (1998) Dopaminergic regulation of extracellular gamma-aminobutyric acid levels in the prefrontal cortex of the rat. $J$ Pharmacol Exp Ther 285, 350-357.

Groenewegen H. J., Berendse H. W., Wolters J. G. and Lohman A. H. (1990) The anatomical relationship of the prefrontal cortex with the striatopallidal system, the thalamus and the amygdala: evidence for a parallel organization. Prog Brain Res 85, 95-116; discussion 116-118.

Groenewegen H. J. and Uylings H. B. (2000) The prefrontal cortex and the integration of sensory, limbic and autonomic information. Prog Brain Res 126, 3-28.

Harmer C. J. and Phillips G. D. (1999) Enhanced dopamine efflux in the amygdala by a predictive, but not a non-predictive, stimulus: facilitation by prior repeated D-amphetamine. Neuroscience 90, 119-130.

Hediger M. A. and Welbourne T. C. (1999) Introduction: glutamate transport, metabolism, and physiological responses. Am J Physiol 277, F477-480.

Hedou G., Feldon J. and Heidbreder C. A. (1999) Effects of cocaine on dopamine in subregions of the rat prefrontal cortex and their efferents to subterritories of the nucleus accumbens. Eur J Pharmacol 372, 143-155.

Heidbreder C. A. and Groenewegen H. J. (2003) The medial prefrontal cortex in the rat: evidence for a dorso-ventral distinction based upon functional and anatomical characteristics. Neurosci Biobehav Rev 27, 555-579.

Heidbreder C. A., Thompson A. C. and Shippenberg T. S. (1996) Role of extracellular dopamine in the initiation and long-term expression of behavioral sensitization to cocaine. J Pharmacol Exp Ther 278, 490-502.

Henry D. J., Greene M. A. and White F. J. (1989) Electrophysiological effects of cocaine in the mesoaccumbens dopamine system: repeated administration. $J$ Pharmacol Exp Ther 251, 833-839.

Henry D. J. and White F. J. (1991) Repeated cocaine administration causes persistent enhancement of D1 dopamine receptor sensitivity within the rat nucleus accumbens. 
J Pharmacol Exp Ther 258, 882-890.

Henry D. J. and White F. J. (1995) The persistence of behavioral sensitization to cocaine parallels enhanced inhibition of nucleus accumbens neurons. $J$ Neurosci 15, 6287-6299.

Hisano S. (2003) Vesicular glutamate transporters in the brain. Anat Sci Int 78, 191-204.

Hooks M. S., Jones G. H., Hemby S. E. and Justice J. B., Jr. (1993) Environmental and pharmacological sensitization: effects of repeated administration of systemic or intra-nucleus accumbens cocaine. Psychopharmacology (Berl) 111, 109-116.

Huang C. C., Lin H. J. and Hsu K. S. (2007) Repeated cocaine administration promotes long-term potentiation induction in rat medial prefrontal cortex. Cereb Cortex 17, 1877-1888.

Huang C. C., Yang P. C., Lin H. J. and Hsu K. S. (2007) Repeated cocaine administration impairs group II metabotropic glutamate receptor-mediated long-term depression in rat medial prefrontal cortex. J Neurosci 27, 2958-2968.

Huntley G. W., Vickers J. C. and Morrison J. H. (1994) Cellular and synaptic localization of NMDA and non-NMDA receptor subunits in neocortex: organizational features related to cortical circuitry, function and disease. Trends Neurosci 17, 536-543.

Hurd Y. L., Weiss F., Koob G. F., And N. E. and Ungerstedt U. (1989) Cocaine reinforcement and extracellular dopamine overflow in rat nucleus accumbens: an in vivo microdialysis study. Brain Res 498, 199-203.

Hurley K. M., Herbert H., Moga M. M. and Saper C. B. (1991) Efferent projections of the infralimbic cortex of the rat. J Comp Neurol 308, 249-276.

Hyman S. E. and Malenka R. C. (2001) Addiction and the brain: the neurobiology of compulsion and its persistence. Nat Rev Neurosci 2, 695-703.

Jackson D. M. and Westlind-Danielsson A. (1994) Dopamine receptors: molecular biology, biochemistry and behavioural aspects. Pharmacol Ther 64, 291-370.

Jay T. M., Burette F. and Laroche S. (1996) Plasticity of the hippocampal-prefrontal cortex synapses. J Physiol Paris 90, 361-366.

Jay T. M., Glowinski J. and Thierry A. M. (1995) Inhibition of hippocampoprefrontal cortex excitatory responses by the mesocortical DA system. Neuroreport 6, $1845-1848$.

Jayaram P. and Steketee J. D. (2004) Effects of repeated cocaine on medial prefrontal cortical GABAB receptor modulation of neurotransmission in the mesocorticolimbic dopamine system. J Neurochem 90, 839-847.

Jayaram P. and Steketee J. D. (2005) Effects of cocaine-induced behavioural sensitization on GABA transmission within rat medial prefrontal cortex. Eur $J$ Neurosci 21, 2035-2039.

Jayaram P. and Steketee J. D. (2006) Cocaine-induced increases in medial prefrontal cortical GABA transmission involves glutamatergic receptors. Eur J Pharmacol 531, 74-79.

Kalivas P. W. (1995) Interactions between dopamine and excitatory amino acids in behavioral sensitization to psychostimulants. Drug Alcohol Depend 37, 95-100. 
Kalivas P. W. (2004) Glutamate systems in cocaine addiction. Curr Opin Pharmacol 4, 23-29.

Kalivas P. W. and Alesdatter J. E. (1993) Involvement of N-methyl-D-aspartate receptor stimulation in the ventral tegmental area and amygdala in behavioral sensitization to cocaine. J Pharmacol Exp Ther 267, 486-495.

Kalivas P. W. and Duffy P. (1990) Effect of acute and daily cocaine treatment on extracellular dopamine in the nucleus accumbens. Synapse 5, 48-58.

Kalivas P. W. and Duffy P. (1990) Effect of acute and daily neurotensin and enkephalin treatments on extracellular dopamine in the nucleus accumbens. $J$ Neurosci 10, 2940-2949.

Kalivas P. W. and Duffy P. (1993) Time course of extracellular dopamine and behavioral sensitization to cocaine. I. Dopamine axon terminals. J Neurosci 13, 266-275.

Kalivas P. W. and Duffy P. (1993) Time course of extracellular dopamine and behavioral sensitization to cocaine. II. Dopamine perikarya. J Neurosci 13, 276-284.

Kalivas P. W. and Duffy P. (1995) D1 receptors modulate glutamate transmission in the ventral tegmental area. J Neurosci 15, 5379-5388.

Kalivas P. W. and Nakamura M. (1999) Neural systems for behavioral activation and reward. Curr Opin Neurobiol 9, 223-227.

Kalivas P. W., Peters J. and Knackstedt L. (2006) Animal models and brain circuits in drug addiction. Mol Interv 6, 339-344.

Kalivas P. W., Pierce R. C., Cornish J. and Sorg B. A. (1998) A role for sensitization in craving and relapse in cocaine addiction. J Psychopharmacol 12, 49-53.

Kalivas P. W., Sorg B. A. and Hooks M. S. (1993) The pharmacology and neural circuitry of sensitization to psychostimulants. Behav Pharmacol 4, 315-334.

Kalivas P. W. and Weber B. (1988) Amphetamine injection into the ventral mesencephalon sensitizes rats to peripheral amphetamine and cocaine. J Pharmacol Exp Ther 245, 1095-1102.

Kane J. K., Hwang Y., Konu O., Loughlin S. E., Leslie F. M. and Li M. D. (2005) Regulation of Homer and group I metabotropic glutamate receptors by nicotine. Eur J Neurosci 21, 1145-1154.

Karasawa J., Yoshimizu T. and Chaki S. (2006) A metabotropic glutamate 2/3 receptor antagonist, MGS0039, increases extracellular dopamine levels in the nucleus accumbens shell. Neurosci Lett 393, 127-130.

Kelly P. H. and Iversen S. D. (1976) Selective 6OHDA-induced destruction of mesolimbic dopamine neurons: abolition of psychostimulant-induced locomotor activity in rats. Eur $J$ Pharmacol 40, 45-56.

Kenny P. J., Gasparini F. and Markou A. (2003) Group II metabotropic and alpha-amino-3-hydroxy-5-methyl-4-isoxazole propionate (AMPA)/kainate glutamate receptors regulate the deficit in brain reward function associated with nicotine withdrawal in rats. J Pharmacol Exp Ther 306, 1068-1076.

Kenny P. J. and Markou A. (2004) The ups and downs of addiction: role of metabotropic glutamate receptors. Trends Pharmacol Sci 25, 265-272. 
Kim J. S., Kornhuber H. H., Brand U. and Menge H. G. (1981) Effects of chronic amphetamine treatment on the glutamate concentration in cerebrospinal fluid and brain: implications for a theory of schizophrenia. Neurosci Lett 24, 93-96.

King G. R., Joyner C., Lee T., Kuhn C. and Ellinwood E. H., Jr. (1992) Intermittent and continuous cocaine administration: residual behavioral states during withdrawal. Pharmacol Biochem Behav 43, 243-248.

Knutson B., Adams C. M., Fong G. W. and Hommer D. (2001) Anticipation of increasing monetary reward selectively recruits nucleus accumbens. J Neurosci 21, RC159.

Koob G. F. and Le Moal M. (1997) Drug abuse: hedonic homeostatic dysregulation. Science 278, 52-58.

Koob G. F. and Le Moal M. (2001) Drug addiction, dysregulation of reward, and allostasis. Neuropsychopharmacology 24, 97-129.

Koob G. F. and Nestler E. J. (1997) The neurobiology of drug addiction. $J$ Neuropsychiatry Clin Neurosci 9, 482-497.

Kornetsky C. and Bain G. (1992) Brain-stimulation reward: a model for the study of the rewarding effects of abused drugs. NIDA Res Monogr 124, 73-93.

Kornetsky C. and Esposito R. U. (1979) Euphorigenic drugs: effects on the reward pathways of the brain. Fed Proc 38, 2473-2476.

Kornetsky C., Esposito R. U., McLean S. and Jacobson J. O. (1979) Intracranial self-stimulation thresholds: a model for the hedonic effects of drugs of abuse. Arch Gen Psychiatry 36, 289-292.

Kushner S. A. and Unterwald E. M. (2001) Chronic cocaine administration decreases the functional coupling of GABA(B) receptors in the rat ventral tegmental area as measured by baclofen-stimulated 35S-GTPgammaS binding. Life Sci 69, 1093-1102.

Kuzmin A. and Johansson B. (2000) Reinforcing and neurochemical effects of cocaine: differences among C57, DBA, and 129 mice. Pharmacol Biochem Behav 65, 399-406.

Lamb R. J., Preston K. L., Schindler C. W., Meisch R. A., Davis F., Katz J. L., Henningfield J. E. and Goldberg S. R. (1991) The reinforcing and subjective effects of morphine in post-addicts: a dose-response study. J Pharmacol Exp Ther 259, 1165-1173.

Lapish C. C., Kroener S., Durstewitz D., Lavin A. and Seamans J. K. (2007) The ability of the mesocortical dopamine system to operate in distinct temporal modes. Psychopharmacology (Berl) 191, 609-625.

Law-Tho D., Hirsch J. C. and Crepel F. (1994) Dopamine modulation of synaptic transmission in rat prefrontal cortex: an in vitro electrophysiological study. Neurosci Res 21, 151-160.

Lepsch L. B., Gonzalo L. A., Magro F. J., Delucia R., Scavone C. and Planeta C. S. (2005) Exposure to chronic stress increases the locomotor response to cocaine and the basal levels of corticosterone in adolescent rats. Addict Biol 10, 251-256.

Lessov C. N. and Phillips T. J. (2003) Cross-sensitization between the locomotor stimulant effects of ethanol and those of morphine and cocaine in mice. Alcohol 
Clin Exp Res 27, 616-627.

Li Y., Hu X. T., Berney T. G., Vartanian A. J., Stine C. D., Wolf M. E. and White F. J. (1999) Both glutamate receptor antagonists and prefrontal cortex lesions prevent induction of cocaine sensitization and associated neuroadaptations. Synapse 34, 169-180.

Li Y., Wolf M. E. and White F. J. (1999) The expression of cocaine sensitization is not prevented by MK-801 or ibotenic acid lesions of the medial prefrontal cortex. Behav Brain Res 104, 119-125.

Lindvall O., Bjorklund A. and Divac I. (1977) Subcortical afferents to the prefrontal cortex: organization of mesencephalic dopaminergic and mediodorsal thalamic projections. Acta Physiol Scand Suppl 452, 35-38.

Liu Q. S., Pu L. and Poo M. M. (2005) Repeated cocaine exposure in vivo facilitates LTP induction in midbrain dopamine neurons. Nature 437, 1027-1031.

Lu W., Chen H., Xue C. J. and Wolf M. E. (1997) Repeated amphetamine administration alters the expression of mRNA for AMPA receptor subunits in rat nucleus accumbens and prefrontal cortex. Synapse 26, 269-280.

Lu W. and Wolf M. E. (1999) Repeated amphetamine administration alters AMPA receptor subunit expression in rat nucleus accumbens and medial prefrontal cortex. Synapse 32, 119-131.

Lu X. Y., Churchill L. and Kalivas P. W. (1997) Expression of D1 receptor mRNA in projections from the forebrain to the ventral tegmental area. Synapse 25, 205-214.

Macenski M. J. and Meisch R. A. (1998) Ratio size and cocaine concentration effects on oral cocaine-reinforced behavior. J Exp Anal Behav 70, 185-201.

Mao L. and Wang J. Q. (2001) Differentially altered mGluR1 and mGluR5 mRNA expression in rat caudate nucleus and nucleus accumbens in the development and expression of behavioral sensitization to repeated amphetamine administration. Synapse 41, 230-240.

Mao L., Yang L., Tang Q., Samdani S., Zhang G. and Wang J. Q. (2005) The scaffold protein Homer 1b/c links metabotropic glutamate receptor 5 to extracellular signal-regulated protein kinase cascades in neurons. J Neurosci 25, 2741-2752.

Markou A., Weiss F., Gold L. H., Caine S. B., Schulteis G. and Koob G. F. (1993) Animal models of drug craving. Psychopharmacology (Berl) 112, 163-182.

McAllister G., Knowles M. R., Ward-Booth S. M., Sinclair H. A., Patel S., Marwood R., Emms F., Patel S., Smith A., Seabrook G. R. and et al. (1995) Functional coupling of human D2, D3, and D4 dopamine receptors in HEK293 cells. J Recept Signal Transduct Res 15, 267-281.

McDonald A. J. (1996) Glutamate and aspartate immunoreactive neurons of the rat basolateral amygdala: colocalization of excitatory amino acids and projections to the limbic circuit. J Comp Neurol 365, 367-379.

McFarland K., Lapish C. C. and Kalivas P. W. (2003) Prefrontal glutamate release into the core of the nucleus accumbens mediates cocaine-induced reinstatement of drug-seeking behavior. J Neurosci 23, 3531-3537.

Melendez R. I. and Kalivas P. W. (2003) Metabotropic glutamate receptor regulation of extracellular glutamate levels in the prefrontal cortex. Ann N Y Acad Sci 1003, 
443-444.

Melendez R. I., Vuthiganon J. and Kalivas P. W. (2005) Regulation of extracellular glutamate in the prefrontal cortex: focus on the cystine glutamate exchanger and group I metabotropic glutamate receptors. J Pharmacol Exp Ther 314, 139-147.

Mello N. K. and Negus S. S. (1996) Preclinical evaluation of pharmacotherapies for treatment of cocaine and opioid abuse using drug self-administration procedures. Neuropsychopharmacology 14, 375-424.

Merlin M. D. (2003) Archaeological evidence for the tradition of psychoactive plant use in the old world. Economic Botany 57, 295-323.

Millan M. J., Newman-Tancredi A., Quentric Y. and Cussac D. (2001) The "selective" dopamine D1 receptor antagonist, $\mathrm{SCH} 23390$, is a potent and high efficacy agonist at cloned human serotonin2C receptors. Psychopharmacology (Berl) 156, 58-62.

Missale C., Nash S. R., Robinson S. W., Jaber M. and Caron M. G. (1998) Dopamine receptors: from structure to function. Physiol Rev 78, 189-225.

Moghaddam B. (2002) Stress activation of glutamate neurotransmission in the prefrontal cortex: implications for dopamine-associated psychiatric disorders. Biol Psychiatry 51, 775-787.

Morishima Y., Miyakawa T., Furuyashiki T., Tanaka Y., Mizuma H. and Nakanishi S. (2005) Enhanced cocaine responsiveness and impaired motor coordination in metabotropic glutamate receptor subtype 2 knockout mice. Proc Natl Acad Sci U S A 102, 4170-4175.

Muly E. C., Maddox M. and Smith Y. (2003) Distribution of mGluR1alpha and mGluR5 immunolabeling in primate prefrontal cortex. J Comp Neurol 467, 521-535.

Nakazawa K., Mikawa S. and Ito M. (1997) Persistent phosphorylation parallels long-term desensitization of cerebellar purkinje cell AMPA-type glutamate receptors. Learn Mem 3, 578-591.

Nasif F. J., Hu X. T. and White F. J. (2005) Repeated cocaine administration increases voltage-sensitive calcium currents in response to membrane depolarization in medial prefrontal cortex pyramidal neurons. J Neurosci 25, 3674-3679.

Nasif F. J., Sidiropoulou K., Hu X. T. and White F. J. (2005) Repeated cocaine administration increases membrane excitability of pyramidal neurons in the rat medial prefrontal cortex. J Pharmacol Exp Ther 312, 1305-1313.

Natochin M., Lester B., Peterson Y. K., Bernard M. L., Lanier S. M. and Artemyev N. O. (2000) AGS3 inhibits GDP dissociation from galpha subunits of the Gi family and rhodopsin-dependent activation of transducin. J Biol Chem 275, 40981-40985.

Navailles S., Moison D., Cunningham K. A. and Spampinato U. (2007) Differential regulation of the mesoaccumbens dopamine circuit by serotonin $2 \mathrm{C}$ receptors in the ventral tegmental area and the nucleus accumbens: an in vivo microdialysis study with cocaine. Neuropsychopharmacology. Apr 11; [Epub ahead of print].

Nestler E. J. (2004) Molecular mechanisms of drug addiction. Neuropharmacology 47 Suppl 1, 24-32.

Nestler E. J., Barrot M. and Self D. W. (2001) DeltaFosB: a sustained molecular switch for addiction. Proc Natl Acad Sci U S A 98, 11042-11046. 
Nestler E. J., Terwilliger R. Z., Walker J. R., Sevarino K. A. and Duman R. S. (1990) Chronic cocaine treatment decreases levels of the $\mathrm{G}$ protein subunits $\mathrm{Gi}$ alpha and Go alpha in discrete regions of rat brain. J Neurochem 55, 1079-1082.

Nutt D. (2006) GABAA receptors: subtypes, regional distribution, and function. $J$ Clin Sleep Med 2, S7-11.

Oades R. D. and Halliday G. M. (1987) Ventral tegmental (A10) system: neurobiology. 1. Anatomy and connectivity. Brain Res 434, 117-165.

Olds J. and Milner P. (1954) Positive reinforcement produced by electrical stimulation of septal area and other regions of rat brain. J Comp Physiol Psychol 47, 419-427.

Olsen R. W., Hanchar H. J., Meera P. and Wallner M. (2007) GABAA receptor subtypes: the "one glass of wine" receptors. Alcohol 41, 201-209.

Ongur D. and Price J. L. (2000) The organization of networks within the orbital and medial prefrontal cortex of rats, monkeys and humans. Cereb Cortex 10, 206-219.

Otani S., Daniel H., Takita M. and Crepel F. (2002) Long-term depression induced by postsynaptic group II metabotropic glutamate receptors linked to phospholipase C and intracellular calcium rises in rat prefrontal cortex. J Neurosci 22, 3434-3444.

Paulson P. E., Camp D. M. and Robinson T. E. (1991) Time course of transient behavioral depression and persistent behavioral sensitization in relation to regional brain monoamine concentrations during amphetamine withdrawal in rats. Psychopharmacology (Berl) 103, 480-492.

Pennartz C. M., Groenewegen H. J. and Lopes da Silva F. H. (1994) The nucleus accumbens as a complex of functionally distinct neuronal ensembles: an integration of behavioural, electrophysiological and anatomical data. Prog Neurobiol 42, 719-761.

Perugini M. and Vezina P. (1994) Amphetamine administered to the ventral tegmental area sensitizes rats to the locomotor effects of nucleus accumbens amphetamine. $J$ Pharmacol Exp Ther 270, 690-696.

Peters J. and Kalivas P. W. (2006) The group II metabotropic glutamate receptor agonist, LY379268, inhibits both cocaine- and food-seeking behavior in rats. Psychopharmacology (Berl) 186, 143-149.

Peterson J. D., Wolf M. E. and White F. J. (2000) Altered responsiveness of medial prefrontal cortex neurons to glutamate and dopamine after withdrawal from repeated amphetamine treatment. Synapse 36, 342-344.

Petralia R. S., Wang Y. X., Niedzielski A. S. and Wenthold R. J. (1996) The metabotropic glutamate receptors, mGluR2 and mGluR3, show unique postsynaptic, presynaptic and glial localizations. Neuroscience 71, 949-976.

Pierce R. C., Bell K., Duffy P. and Kalivas P. W. (1996) Repeated cocaine augments excitatory amino acid transmission in the nucleus accumbens only in rats having developed behavioral sensitization. J Neurosci 16, 1550-1560.

Pierce R. C., Born B., Adams M. and Kalivas P. W. (1996) Repeated intra-ventral tegmental area administration of SKF-38393 induces behavioral and neurochemical sensitization to a subsequent cocaine challenge. J Pharmacol Exp Ther 278, 384-392.

Pierce R. C. and Kalivas P. W. (1997) A circuitry model of the expression of 
behavioral sensitization to amphetamine-like psychostimulants. Brain Res Brain Res Rev 25, 192-216.

Pierce R. C., Reeder D. C., Hicks J., Morgan Z. R. and Kalivas P. W. (1998) Ibotenic acid lesions of the dorsal prefrontal cortex disrupt the expression of behavioral sensitization to cocaine. Neuroscience 82, 1103-1114.

Pirot S., Glowinski J. and Thierry A. M. (1996) Mediodorsal thalamic evoked responses in the rat prefrontal cortex: influence of the mesocortical DA system. Neuroreport 7, 1437-1441.

Pirot S., Godbout R., Mantz J., Tassin J. P., Glowinski J. and Thierry A. M. (1992) Inhibitory effects of ventral tegmental area stimulation on the activity of prefrontal cortical neurons: evidence for the involvement of both dopaminergic and GABAergic components. Neuroscience 49, 857-865.

Pirot S., Jay T. M., Glowinski J. and Thierry A. M. (1994) Anatomical and electrophysiological evidence for an excitatory amino acid pathway from the thalamic mediodorsal nucleus to the prefrontal cortex in the rat. Eur J Neurosci 6, 1225-1234.

Porrino L. J., Esposito R. U., Seeger T. F., Crane A. M., Pert A. and Sokoloff L. (1984) Metabolic mapping of the brain during rewarding self-stimulation. Science 224, 306-309.

Post R. M. (1980) Intermittent versus continuous stimulation: effect of time interval on the development of sensitization or tolerance. Life Sci 26, 1275-1282.

Post R. M. and Kopanda R. T. (1976) Cocaine, kindling, and psychosis. Am J Psychiatry 133, 627-634.

Prasad B. M., Ulibarri C. and Sorg B. A. (1998) Stress-induced cross-sensitization to cocaine: effect of adrenalectomy and corticosterone after short- and long-term withdrawal. Psychopharmacology (Berl) 136, 24-33.

Przegalinski E., Czepiel K., Nowak E., Dlaboga D. and Filip M. (2003) Withdrawal from chronic cocaine up-regulates 5-HT1B receptors in the rat brain. Neurosci Lett 351, 169-172.

Reid M. S., Hsu K., Jr. and Berger S. P. (1997) Cocaine and amphetamine preferentially stimulate glutamate release in the limbic system: studies on the involvement of dopamine. Synapse 27, 95-105.

Risinger F. O., Bormann N. M. and Oakes R. A. (1996) Reduced sensitivity to ethanol reward, but not ethanol aversion, in mice lacking 5-HT1B receptors. Alcohol Clin Exp Res 20, 1401-1405.

Robinson T. E. and Becker J. B. (1986) Enduring changes in brain and behavior produced by chronic amphetamine administration: a review and evaluation of animal models of amphetamine psychosis. Brain Res 396, 157-198.

Robinson T. E. and Berridge K. C. (1993) The neural basis of drug craving: an incentive-sensitization theory of addiction. Brain Res Brain Res Rev 18, 247-291.

Robinson T. E. and Berridge K. C. (2000) The psychology and neurobiology of addiction: an incentive-sensitization view. Addiction 95 Suppl 2, S91-117.

Robinson T. E. and Berridge K. C. (2001) Incentive-sensitization and addiction. Addiction 96, 103-114. 
Robinson T. E. and Berridge K. C. (2003) Addiction. Annu Rev Psychol 54, 25-53.

Rossetti Z. L., Marcangione C. and Wise R. A. (1998) Increase of extracellular glutamate and expression of Fos-like immunoreactivity in the ventral tegmental area in response to electrical stimulation of the prefrontal cortex. J Neurochem 70, 1503-1512.

Roth R. H. (1984) CNS dopamine autoreceptors: distribution, pharmacology, and function. Ann N Y Acad Sci 430, 27-53.

Saal D., Dong Y., Bonci A. and Malenka R. C. (2003) Drugs of abuse and stress trigger a common synaptic adaptation in dopamine neurons. Neuron 37, 577-582.

Sanchez C. J., Bailie T. M., Wu W. R., Li N. and Sorg B. A. (2003) Manipulation of dopamine d1-like receptor activation in the rat medial prefrontal cortex alters stress- and cocaine-induced reinstatement of conditioned place preference behavior. Neuroscience 119, 497-505.

Sanchis-Segura C. and Spanagel R. (2006) Behavioural assessment of drug reinforcement and addictive features in rodents: an overview. Addict Biol 11, 2-38.

Schiffmann S. N., Lledo P. M. and Vincent J. D. (1995) Dopamine D1 receptor modulates the voltage-gated sodium current in rat striatal neurones through a protein kinase A. J Physiol 483 (Pt 1), 95-107.

Schmidt W. J. and Beninger R. J. (2006) Behavioural sensitization in addiction, schizophrenia, Parkinson's disease and dyskinesia. Neurotox Res 10, 161-166.

Schoepfer R., Monyer H., Sommer B., Wisden W., Sprengel R., Kuner T., Lomeli H., Herb A., Kohler M., Burnashev N. and et al. (1994) Molecular biology of glutamate receptors. Prog Neurobiol 42, 353-357.

Schoffelmeer A. N., De Vries T. J., Wardeh G., van de Ven H. W. and Vanderschuren L. J. (2002) Psychostimulant-induced behavioral sensitization depends on nicotinic receptor activation. $J$ Neurosci 22, 3269-3276.

Schultz W. (1998) Predictive reward signal of dopamine neurons. J Neurophysiol 80, $1-27$.

Schultz W. (2000) Multiple reward signals in the brain. Nat Rev Neurosci 1, 199-207.

Schultz W., Tremblay L. and Hollerman J. R. (1998) Reward prediction in primate basal ganglia and frontal cortex. Neuropharmacology 37, 421-429.

Seamans J. K. and Yang C. R. (2004) The principal features and mechanisms of dopamine modulation in the prefrontal cortex. Prog Neurobiol 74, 1-58.

Sesack S. R., Deutch A. Y., Roth R. H. and Bunney B. S. (1989) Topographical organization of the efferent projections of the medial prefrontal cortex in the rat: an anterograde tract-tracing study with Phaseolus vulgaris leucoagglutinin. J Comp Neurol 290, 213-242.

Sesack S. R. and Pickel V. M. (1992) Prefrontal cortical efferents in the rat synapse on unlabeled neuronal targets of catecholamine terminals in the nucleus accumbens septi and on dopamine neurons in the ventral tegmental area. J Comp Neurol 320, 145-160.

Sesack S. R., Snyder C. L. and Lewis D. A. (1995) Axon terminals immunolabeled for dopamine or tyrosine hydroxylase synapse on GABA-immunoreactive dendrites in rat and monkey cortex. J Comp Neurol 363, 264-280. 
Shigeri Y., Seal R. P. and Shimamoto K. (2004) Molecular pharmacology of glutamate transporters, EAATs and VGLUTs. Brain Res Brain Res Rev 45, 250-265.

Smith J. E., Koves T. R. and Co C. (2003) Brain neurotransmitter turnover rates during rat intravenous cocaine self-administration. Neuroscience 117, 461-475.

Solomon R. L. and Corbit J. D. (1973) An opponent-process theory of motivation. II. Cigarette addiction. J Abnorm Psychol 81, 158-171.

Sorg B. A. (1992) Mesocorticolimbic dopamine systems: cross-sensitization between stress and cocaine. Ann N Y Acad Sci 654, 136-144.

Sorg B. A., Chen S. Y. and Kalivas P. W. (1993) Time course of tyrosine hydroxylase expression after behavioral sensitization to cocaine. J Pharmacol Exp Ther 266, 424-430.

Sorg B. A., Davidson D. L., Kalivas P. W. and Prasad B. M. (1997) Repeated daily cocaine alters subsequent cocaine-induced increase of extracellular dopamine in the medial prefrontal cortex. J Pharmacol Exp Ther 281, 54-61.

Sorg B. A., Li N. and Wu W. R. (2001) Dopamine D1 receptor activation in the medial prefrontal cortex prevents the expression of cocaine sensitization. $J$ Pharmacol Exp Ther 297, 501-508.

Steketee J. D. (1997) Intra-ventral tegmental area administration of H7 delays, but does not prevent the development of cocaine-induced sensitization. Brain Res Bull 43, 565-571.

Steketee J. D. (1998) Injection of SCH 23390 into the ventral tegmental area blocks the development of neurochemical but not behavioral sensitization to cocaine. Behav Pharmacol 9, 69-76.

Steketee J. D. (1998) Repeated injection of GBR 12909, but not cocaine or WIN 35,065-2, into the ventral tegmental area induces behavioral sensitization. Behav Brain Res 97, 39-48.

Steketee J. D. (2003) Neurotransmitter systems of the medial prefrontal cortex: potential role in sensitization to psychostimulants. Brain Res Brain Res Rev 41, 203-228.

Steketee J. D. (2005) Cortical mechanisms of cocaine sensitization. Crit Rev Neurobiol 17, 69-86.

Steketee J. D. and Beyer C. E. (2005) Injections of baclofen into the ventral medial prefrontal cortex block the initiation, but not the expression, of cocaine sensitization in rats. Psychopharmacology (Berl) 180, 352-358.

Steketee J. D., Rowe L. A. and Chandler L. J. (1998) The effects of acute and repeated cocaine injections on protein kinase $\mathrm{C}$ activity and isoform levels in dopaminergic brain regions. Neuropharmacology 37, 339-347.

Steketee J. D., Sorg B. A. and Kalivas P. W. (1992) The role of the nucleus accumbens in sensitization to drugs of abuse. Prog Neuropsychopharmacol Biol Psychiatry 16, 237-246.

Steketee J. D., Striplin C. D., Murray T. F. and Kalivas P. W. (1991) Possible role for G-proteins in behavioral sensitization to cocaine. Brain Res 545, 287-291.

Steketee J. D. and Walsh T. J. (2005) Repeated injections of sulpiride into the medial prefrontal cortex induces sensitization to cocaine in rats. Psychopharmacology 
(Berl) 179, 753-760.

Stellar J. and Stellar E. (1985) Neurobiology of Reward and Motivation. Springer-Verlag, New York.

Stephans S. E. and Yamamoto B. Y. (1995) Effect of repeated methamphetamine administrations on dopamine and glutamate efflux in rat prefrontal cortex. Brain Res 700, 99-106.

Surmeier D. J., Bargas J., Hemmings H. C., Jr., Nairn A. C. and Greengard P. (1995) Modulation of calcium currents by a D1 dopaminergic protein kinase/phosphatase cascade in rat neostriatal neurons. Neuron 14, 385-397.

Svensson L., Zhang J., Johannessen K. and Engel J. A. (1994) Effect of local infusion of glutamate analogues into the nucleus accumbens of rats: an electrochemical and behavioural study. Brain Res 643, 155-161.

Swanson C. J., Baker D. A., Carson D., Worley P. F. and Kalivas P. W. (2001) Repeated cocaine administration attenuates group I metabotropic glutamate receptor-mediated glutamate release and behavioral activation: a potential role for Homer. J Neurosci 21, 9043-9052.

Taber M. T. and Fibiger H. C. (1993) Electrical stimulation of the medial prefrontal cortex increases dopamine release in the striatum. Neuropsychopharmacology $\mathbf{9}$, 271-275.

Takagishi M. and Chiba T. (1991) Efferent projections of the infralimbic (area 25) region of the medial prefrontal cortex in the rat: an anterograde tracer PHA-L study. Brain Res 566, 26-39.

Tang L., Todd R. D. and O'Malley K. L. (1994) Dopamine D2 and D3 receptors inhibit dopamine release. J Pharmacol Exp Ther 270, 475-479.

Tang X. C. and Kalivas P. W. (2003) Bidirectional modulation of cystine/glutamate exchanger activity in cultured cortical astrocytes. Ann N Y Acad Sci 1003, 472-475.

Tappe A. and Kuner R. (2006) Regulation of motor performance and striatal function by synaptic scaffolding proteins of the Homer1 family. Proc Natl Acad Sci U S A 103, 774-779.

Thierry A. M., Blanc G., Sobel A., Stinus L. and Golwinski J. (1973) Dopaminergic terminals in the rat cortex. Science 182, 499-501.

Thomas M. J., Beurrier C., Bonci A. and Malenka R. C. (2001) Long-term depression in the nucleus accumbens: a neural correlate of behavioral sensitization to cocaine. Nat Neurosci 4, 1217-1223.

Todtenkopf M. S., Marcus J. F., Portoghese P. S. and Carlezon W. A., Jr. (2004) Effects of kappa-opioid receptor ligands on intracranial self-stimulation in rats. Psychopharmacology (Berl) 172, 463-470.

Tolliver B. K., Ho L. B., Fox L. M. and Berger S. P. (1999) Necessary role for ventral tegmental area adenylate cyclase and protein kinase $\mathrm{A}$ in induction of behavioral sensitization to intraventral tegmental area amphetamine. J Pharmacol Exp Ther 289, 38-47.

Trantham-Davidson H., Neely L. C., Lavin A. and Seamans J. K. (2004) Mechanisms underlying differential D1 versus D2 dopamine receptor regulation of inhibition in prefrontal cortex. J Neurosci 24, 10652-10659. 
Tzschentke T. M. (1998) Measuring reward with the conditioned place preference paradigm: a comprehensive review of drug effects, recent progress and new issues. Prog Neurobiol 56, 613-672.

Tzschentke T. M. (2000) The medial prefrontal cortex as a part of the brain reward system. Amino Acids 19, 211-219.

Tzschentke T. M. (2001) Pharmacology and behavioral pharmacology of the mesocortical dopamine system. Prog Neurobiol 63, 241-320.

Tzschentke T. M. and Schmidt W. J. (1998) The development of cocaine-induced behavioral sensitization is affected by discrete quinolinic acid lesions of the prelimbic medial prefrontal cortex. Brain Res 795, 71-76.

Tzschentke T. M. and Schmidt W. J. (2000) Differential effects of discrete subarea-specific lesions of the rat medial prefrontal cortex on amphetamine- and cocaine-induced behavioural sensitization. Cereb Cortex 10, 488-498.

Undie A. S. and Friedman E. (1990) Stimulation of a dopamine D1 receptor enhances inositol phosphates formation in rat brain. J Pharmacol Exp Ther 253, 987-992.

Ungless M. A., Whistler J. L., Malenka R. C. and Bonci A. (2001) Single cocaine exposure in vivo induces long-term potentiation in dopamine neurons. Nature 411, 583-587.

Valentijn J. A., Vaudry H. and Cazin L. (1993) Multiple control of calcium channel gating by dopamine D2 receptors in frog pituitary melanotrophs. Ann N Y Acad Sci 680, 211-228.

Vallar L., Muca C., Magni M., Albert P., Bunzow J., Meldolesi J. and Civelli O. (1990) Differential coupling of dopaminergic D2 receptors expressed in different cell types. Stimulation of phosphatidylinositol 4,5-bisphosphate hydrolysis in LtK- fibroblasts, hyperpolarization, and cytosolic-free $\mathrm{Ca} 2+$ concentration decrease in $\mathrm{GH} 4 \mathrm{C} 1$ cells. J Biol Chem 265, 10320-10326.

Vanderschuren L. J. and Kalivas P. W. (2000) Alterations in dopaminergic and glutamatergic transmission in the induction and expression of behavioral sensitization: a critical review of preclinical studies. Psychopharmacology (Berl) 151, 99-120.

Vanderschuren L. J., Schmidt E. D., De Vries T. J., Van Moorsel C. A., Tilders F. J. and Schoffelmeer A. N. (1999) A single exposure to amphetamine is sufficient to induce long-term behavioral, neuroendocrine, and neurochemical sensitization in rats. J Neurosci 19, 9579-9586.

Vezina P. (1993) Amphetamine injected into the ventral tegmental area sensitizes the nucleus accumbens dopaminergic response to systemic amphetamine: an in vivo microdialysis study in the rat. Brain Res 605, 332-337.

Vezina P. (1996) D1 dopamine receptor activation is necessary for the induction of sensitization by amphetamine in the ventral tegmental area. J Neurosci 16, 2411-2420.

Vezina P., Kalivas P. W. and Stewart J. (1987) Sensitization occurs to the locomotor effects of morphine and the specific mu opioid receptor agonist, DAGO, administered repeatedly to the ventral tegmental area but not to the nucleus accumbens. Brain Res 417, 51-58. 
Vezina P. and Kim J. H. (1999) Metabotropic glutamate receptors and the generation of locomotor activity: interactions with midbrain dopamine. Neurosci Biobehav Rev 23, 577-589.

Vezina P. and Stewart J. (1989) The effect of dopamine receptor blockade on the development of sensitization to the locomotor activating effects of amphetamine and morphine. Brain Res 499, 108-120.

Vezina P. and Stewart J. (1990) Amphetamine administered to the ventral tegmental area but not to the nucleus accumbens sensitizes rats to systemic morphine: lack of conditioned effects. Brain Res 516, 99-106.

Wang J. and O'Donnell P. (2001) D(1) dopamine receptors potentiate nmda-mediated excitability increase in layer V prefrontal cortical pyramidal neurons. Cereb Cortex 11, 452-462.

Watkins J. C. (2000) l-glutamate as a central neurotransmitter: looking back. Biochem Soc Trans 28, 297-309.

Weiss F., Paulus M. P., Lorang M. T. and Koob G. F. (1992) Increases in extracellular dopamine in the nucleus accumbens by cocaine are inversely related to basal levels: effects of acute and repeated administration. $J$ Neurosci 12, 4372-4380.

White F. J. (1996) Synaptic regulation of mesocorticolimbic dopamine neurons. Annu Rev Neurosci 19, 405-436.

White F. J., Hu X. T., Zhang X. F. and Wolf M. E. (1995) Repeated administration of cocaine or amphetamine alters neuronal responses to glutamate in the mesoaccumbens dopamine system. J Pharmacol Exp Ther 273, 445-454.

White N. M. (1996) Addictive drugs as reinforcers: multiple partial actions on memory systems. Addiction 91, 921-949; discussion 951-965.

WHO (1957) WHO Tech. Rep. Ser., No. 116 Geneva. World Health Organization Expert Committee on Addiction-Producing Drugs.

http://www.unodc.org/unodc/en/bulletin/bulletin_1957-01-01_1_page007.html. October 15, 2007

WHO (1964) WHO Tech. Rep. Ser., No. 116 Geneva. World Health Organization Expert Committee on Addiction-Producing Drugs.

http://www.unodc.org/unodc/en/bulletin/bulletin_1964-01-01_2_page006.html. October 15, 2007

Williams J. M. and Steketee J. D. (2004) Characterization of dopamine transport in crude synaptosomes prepared from rat medial prefrontal cortex. J Neurosci Methods 137, 161-165.

Williams J. M. and Steketee J. D. (2004) Cocaine increases medial prefrontal cortical glutamate overflow in cocaine-sensitized rats: a time course study. Eur J Neurosci 20, 1639-1646.

Williams J. M. and Steketee J. D. (2005) Effects of repeated cocaine on the release and clearance of dopamine within the rat medial prefrontal cortex. Synapse 55, 98-109.

Williams J. M. and Steketee J. D. (2005) Time-dependent effects of repeated cocaine administration on dopamine transmission in the medial prefrontal cortex.

Neuropharmacology 48, 51-61. 
Wilson J. M. and Kish S. J. (1996) The vesicular monoamine transporter, in contrast to the dopamine transporter, is not altered by chronic cocaine self-administration in the rat. $J$ Neurosci 16, 3507-3510.

Wise R. A. (1996) Addictive drugs and brain stimulation reward. Annu Rev Neurosci 19, 319-340.

Wise R. A. (1998) Drug-activation of brain reward pathways. Drug Alcohol Depend 51, 13-22.

Wise R. A. and Bozarth M. A. (1987) A psychomotor stimulant theory of addiction. Psychol Rev 94, 469-492.

Wolf M. E. (1998) The role of excitatory amino acids in behavioral sensitization to psychomotor stimulants. Prog Neurobiol 54, 679-720.

Wolf M. E. and Roth R. H. (1987) Dopamine neurons projecting to the medial prefrontal cortex possess release-modulating autoreceptors. Neuropharmacology 26, 1053-1059.

Wolf M. E. and Roth R. H. (1990) Autoreceptor regulation of dopamine synthesis. Ann N Y Acad Sci 604, 323-343.

Wu W. R., Li N. and Sorg B. A. (2003) Prolonged effects of repeated cocaine on medial prefrontal cortex dopamine response to cocaine and a stressful predatory odor challenge in rats. Brain Res 991, 232-239.

Xi Z. X., Ramamoorthy S., Baker D. A., Shen H., Samuvel D. J. and Kalivas P. W. (2002) Modulation of group II metabotropic glutamate receptor signaling by chronic cocaine. J Pharmacol Exp Ther 303, 608-615.

Yoon H. S., Kim S., Park H. K. and Kim J. H. (2007) Microinjection of CART peptide 55-102 into the nucleus accumbens blocks both the expression of behavioral sensitization and ERK phosphorylation by cocaine. Neuropharmacology 53, 344-351.

Zhang G. C., Mao L. M., Liu X. Y. and Wang J. Q. (2007) Long-lasting up-regulation of orexin receptor type 2 protein levels in the rat nucleus accumbens after chronic cocaine administration. J Neurochem.

Zhang K., Tarazi F. I., Campbell A. and Baldessarini R. J. (2000) GABA(B) receptors: altered coupling to G-proteins in rats sensitized to amphetamine. Neuroscience 101, $5-10$.

Zhang X., Lee T. H., Davidson C., Lazarus C., Wetsel W. C. and Ellinwood E. H. (2007) Reversal of cocaine-induced behavioral sensitization and associated phosphorylation of the NR2B and GluR1 subunits of the NMDA and AMPA receptors. Neuropsychopharmacology 32, 377-387.

Zhang X. F., Hu X. T., White F. J. and Wolf M. E. (1997) Increased responsiveness of ventral tegmental area dopamine neurons to glutamate after repeated administration of cocaine or amphetamine is transient and selectively involves AMPA receptors. $J$ Pharmacol Exp Ther 281, 699-706. 


\section{VITA}

Xiaohu Xie was born in Nanjing, China on Feberary 19, 1979. After graduating from the high school affiliated to Nanjing Normal University (Nanjing) in 1997, he attended Nanjing University in Nanjing, China, where he received his Bachelor of Science degree in Biopharmaceutics in September of 2001. Later on, he worked as a Research Assistant in the Center of Molecular Pharmacology in School of Biopharmaceutical Sciences at China Pharmaceutical University. In the Fall of 2003, he entered the graduate program in the Department of Pharmacology at the University of Tennessee Health Science Center. In the Summer of 2004, he joined the laboratory of Dr. Jeffery Steketee. He received a Doctor of Philosophy degree in Pharmacology in December of 2007.

He is currently employed as Postdoctoral Researcher in the Department of Psychology at University of North Carolina at Chapel Hill. 